OPEN ACCESS

Edited by:

Michat Tomczyk,

Medical University of Bialystok, Poland

Reviewed by:

Maria Cristina Duarte,

University of Lisbon, Portugal

Maurice D Awouafack,

University of Dschang, Cameroon

${ }^{*}$ Correspondence:

Sylvin Benjamin Ateba

s.benjaminateba@gmail.com

Liselotte Krenn

liselotte.krenn@univie.ac.at

tORCID:

Sylvin Benjamin Ateba orcid.org/0000-0003-1198-9347

Specialty section:

This article was submitted to

Ethnopharmacology,

a section of the journal

Frontiers in Pharmacology

Received: 13 December 2020

Accepted: 17 February 2021

Published: 07 May 2021

Citation:

Ateba SB, Njamen D and Krenn L

(2021) The Genus Eriosema

(Fabaceae): From the

Ethnopharmacology to an Evidence-

Based Phytotherapeutic Perspective?

Front. Pharmacol. 12:641225

doi: 10.3389/fphar.2021.641225

\section{The Genus Eriosema (Fabaceae): From the Ethnopharmacology to an Evidence-Based Phytotherapeutic Perspective?}

\author{
Sylvin Benjamin Ateba ${ }^{1 * t}$, Dieudonné Njamen ${ }^{2}$ and Liselotte Krenn ${ }^{3 *}$ \\ ${ }^{1}$ Department of Biology of Animal Organisms, Faculty of Science, University of Douala, Douala, Cameroon, ${ }^{2}$ Laboratory of Animal \\ Physiology, Department of Animal Biology and Physiology, Faculty of Science, University of Yaoundé I, Yaoundé, Cameroon, \\ ${ }^{3}$ Department of Pharmacognosy, University of Vienna, Vienna, Austria
}

The genus Eriosema (Fabaceae) includes approximately 150 species widely distributed across tropical and subtropical regions of the world (Africa, Neotropics, Asia and Australia). Throughout these regions, several species are used since centuries in different traditional medicinal systems, while others are used as food or food supplement. The present review attempts to critically summarize current information concerning the uses, phytochemistry and pharmacology of the Eriosema genus and to evaluate the therapeutic potential. The information published in English and French (up to September 2020) on ethnopharmacology or traditional uses, chemistry, pharmacology and toxicology of Eriosema genus was collected from electronic databases [SciFinder, PubMed, Google, Google Scholar, Scopus, Web of Science, Prelude Medicinal Plants-http://www. ethnopharmacologia.org/recherche-dans-prelude/?plant, The Plant List (http://www. theplantlist.org/), POWO (http://powo.science.kew.org/) and IUCN Red List Categories (https://www.iucnredlist.org/)], conference proceedings, books, M.Sc. and Ph.D. dissertations. The information retrieved on the ethnomedicinal indications of Eriosema genus allowed to list 25 species ( $16.6 \%$ of the genus). The majority of uses is recorded from Africa. Phytochemical analyses of 8 species led to the identification and/or isolation of 107 compounds, with flavonoids (69.2\%), chromones (7.5\%) and benzoic acid derivatives (3.7\%) as the main chemical classes. Pharmacological investigations with crude extracts and isolated compounds showed a broad range of activities including aphrodisiac, estrogenic, anti-osteoporosis, hypolipidemic, anti-diabetic, anti-diarrheal, anti-microbial, anti-oxidant, anthelmintic, anti-cancer, and acetylcholinesterase inhibitory activities. Despite the low number of Eriosema species tested, there is convincing evidence in vitro and in vivo studies validating some traditional and ethnobotanical uses. However, the utility of several of the described uses has not yet been confirmed in pharmacological studies. Reviewed data could serve as a reference tool and preliminary information for advanced research on Eriosema species.

Keywords: Eriosema, Fabaceae, pharmacological activities, phytochemistry, ethnopharmacology, toxicology 


\section{INTRODUCTION}

Eriosema (Fabaceae), with approximately 150 species of shrubs, shrublets and herbs, is the second-largest genus of the Cajaninae subtribe (Ma et al., 1995; LPWG, 2017). This monophyletic clade originated in the late Miocene and its diversification occurred in parallel with the savanna biome expansion around the world (Cândido et al., 2020). The current centers of diversity and endemism of Eriosema species include the grasslands, wooded savanna and waste places of Africa (West, Central, East and South Africa), Central America (from Mexico to northern Argentina, except for Chile), Southeast Asia and Northern Australia (Wu, 1991; Schrire et al., 2005; Cândido et al., 2019). The African species are most abundant (nearly 110 species) and the first diverging evolution suggests an African origin of the genus (Cândido et al., 2020). The study by Cândido et al. (2019) recorded 35 Eriosema species in Brazil, which encompasses $85 \%$ of the diversity of the genus in the Americas (35 out of 41 species).

Throughout this pantropical region, several Eriosema species are intensively used in traditional medicine and for nonmedicinal purposes (e.g. food/vegetables, tooth brush). Based on the traditional uses, phytochemical, pharmacological and toxicological investigations of different Eriosema species have been performed. Nevertheless, till now only few reviews dealing with Eriosema species have been published. The studies by Cândido et al. (2019, 2020), respectively, focused on the taxonomic synopsis of Eriosema in Brazil and molecular phylogenetic insights into the evolution of Eriosema. In 2015, Awouafack et al. demonstrated that Eriosema species represent a rich source of flavonoids with interesting pharmacological activities. In this review, authors compiled a total of 52 flavonoids (isoflavones, dihydroflavanols, isoflavanones, flavanols, flavanones, and dihydrochalcones) isolated from Eriosema species. However, the pharmacological properties are not limited to the flavonoids. In the literature, compounds from other chemical classes as well as plant extracts (more easily available and affordable than isolated compounds) demonstrated pharmacological properties. More recently, the review of Kleynhans et al. (2020) focused on Eriosema kraussianum Meisn. None of these reviews presented the global overview on the traditional uses, phytochemistry, pharmacological properties and toxicological evaluation of this genus. Accordingly, the present review attempts to critically summarize the available information on the traditional uses, phytochemistry, and pharmacological activities as well as safety evaluation of the genus Eriosema and, therefore to evaluate the data for potential phytotherapeutic approaches.

\section{SEARCH STRATEGY AND TERMS USED}

The information on the genus Eriosema published up to September 2020 in English and French was collected from different electronic data bases (SciFinder including Chemical Abstracts and Medline, PubMed, Google, Google Scholar, Scopus, Web of Science), conference proceedings, books, M.Sc. and Ph.D. dissertations. The keywords "Eriosema" in conjunction with 'traditional uses' or 'ethnopharmacology,' 'phytochemistry,' 'pharmacology,' and 'toxicology' were used. In addition, Prelude Medicinal Plants (http://www.ethnopharmacologia.org/ recherche-dans-prelude/?plant), The Plant List (http://www. theplantlist.org/) and POWO (http://powo.science.kew.org/) were explored for the plant's traditional uses and scientific names, while information on the global extinction risk status of Eriosema species was gathered from the IUCN (International Union for Conservation of Nature) Red List Categories (https:// www.iucnredlist.org/).

\section{TRADITIONAL USES AND ETHNOPHARMACOLOGY OF ERIOSEMA SPECIES}

Since centuries, Eriosema species have widely been traditionally used as household remedies against various human ailments. Accordingly, in the pharmaceutical landscape this genus may represent a remarkable source of promising substances. From the literature review, various medicinal and contemporary uses associated with this genus are summarized in Table $\mathbf{1}$.

According to South African Zulu traditional health practitioners, the roots of several Eriosema species such as Eriosema kraussianum N. E. Br., Eriosema salignum E. Mey. and Eriosema cordatum E. Mey., known under the indigenous umbrella name of $u$ Bangalala, are effective to cure or alleviate erectile dysfunction (ED) and/or impotence (Bryant, 1966; Hutchings et al., 1996; Ojewole et al., 2007; Drewes et al., 2013). In Southern and Eastern Africa, they are also used as expectorants and diuretics (Watt and Breyer-Brandwijk, 1962). In case of impotence, hot milk infusions of the plants' roots and/ or pounded boiled root decoctions are taken at small doses twice a day (Hulme, 1954; Bryant, 1966). Studies reported that the roots of E. cordatum are used for male (Hutchings et al., 1996) and female (Bryant, 1966) infertilities and as constituent of Imbiza ephuzwato, a Zulu traditional herbal medicine (Van Wyk et al., 1997). This mixture is used as tonic, against anxiety, for clearing skin conditions, to treat diabetes mellitus, kidney and urinary infections, tonsillitis, pneumonia, constipation, stomach and back pains, for the improvement of high blood pressure, to boost energy, vitality and sexual activity as well as in the prevention of arthritis (Ndhlala et al., 2011).

In Angola, fresh leaves of Eriosema glomeratum (Guill. \& Perr.) Hook. f. are eaten as food/vegetables and administered against diarrhea and dysentery (Göhre et al., 2016; Lautenschläger et al., 2018). In Benin, they are used against diabetes (Lawin et al., 2015), while the leafy stem is claimed to promote the closure of fotannel (Adomou et al., 2012). The roots are applied in Burkina Faso against hernia (Ouoba et al., 2006). In the Bakola Pygmy tribe, the whole plant is used for many purposes including pulmonary troubles, nasopharyngeal infections, leprosy and venereal diseases (Verdcourt, 1970).

Indians around Kunana (Venezuela) use the root decoction of Eriosema rufum G. Don. against sterility in women and to accelerate delivery in childbirth (Morton, 1981). 
TABLE 1 | Ethnomedicinal indications and local uses of Eriosema species based on the literature data review.

\begin{tabular}{|c|c|c|c|c|c|}
\hline Species & Parts used & Location & $\begin{array}{l}\text { Local and } \\
\text { traditional uses }\end{array}$ & Preparation & References \\
\hline \multirow[t]{2}{*}{ Eriosema affine De Wild. } & Roots & $\begin{array}{l}\text { Angola (Bié } \\
\text { province) }\end{array}$ & Malaria, stomach pain, diarrhea & Decoction & Novotna et al. (2020) \\
\hline & & Angola & $\begin{array}{l}\text { Prevention of abortion, chest pain, } \\
\text { malaria, vomiting, nightmares, } \\
\text { epilepsy, flu }\end{array}$ & Not specified & Bossard (1996) \\
\hline $\begin{array}{l}\text { Eriosema benthamianum Mart. } \\
\text { ex Benth. }\end{array}$ & Roots & Brazil & Inflammation & Not specified & Hirschhorn (1982) \\
\hline $\begin{array}{l}\text { Eriosema burkei Benth. ex Harv. } \\
\& \text { Sond. }\end{array}$ & Roots & Malawi & Pain, lymphoid disorders & Water extract & Msonthi and Magombo (1983) \\
\hline $\begin{array}{l}\text { Eriosema campestre var. } \\
\text { macrophyllum (Gear) Fortunato. }\end{array}$ & Roots & $\begin{array}{l}\text { Brazil (Brazilian } \\
\text { Cerrado) }\end{array}$ & $\begin{array}{l}\text { Inflammatory diseases e.g. } \\
\text { inflammatory skin disorders such } \\
\text { as psoriasis }\end{array}$ & Decoction & Santos et al. (2016) \\
\hline \multirow{6}{*}{$\begin{array}{l}\text { Eriosema chinense Vogel (syn. } \\
\text { Eriosema himalaicum H.Ohashi } \\
\text { or Eriosema tuberosum (Ham.) } \\
\text { Wang et Tang) }\end{array}$} & Roots & $\begin{array}{l}\text { Northern } \\
\text { Australia, China, } \\
\text { North East of India }\end{array}$ & Food/vegetable, diarrhea & $\begin{array}{l}\text { Consumption of fresh } \\
\text { roots }\end{array}$ & $\begin{array}{l}\text { Neogi et al. (1989); Martin and Ryan } \\
\text { (2004); Prasad et al. (2013c) }\end{array}$ \\
\hline & & China & Diarrhea, orchitis, detoxification & Not specified & Kunming Institute of Botany (1979) \\
\hline & & Thailand & Tonic & $\begin{array}{l}\text { Consumption of fresh } \\
\text { roots }\end{array}$ & Thongnest et al. (2013) \\
\hline & $\begin{array}{l}\text { Seeds, } \\
\text { leaves }\end{array}$ & India (Meghalaya) & $\begin{array}{l}\text { Diarrhea, wounds, astringent, } \\
\text { diuretic, tonic, cold sweats, and } \\
\text { parturition (promoting discharge of } \\
\text { lochia) }\end{array}$ & Decoction & $\begin{array}{l}\text { Prasad et al. (2013c), Ashraf and } \\
\text { Borthakur (2005), Laloo and } \\
\text { Hemalatha (2011), Thongnest et al. } \\
\text { (2013) }\end{array}$ \\
\hline & Seeds & $\begin{array}{l}\text { Thailand (central } \\
\text { region of } \\
\text { Myanmar) }\end{array}$ & $\begin{array}{l}\text { Scrofula, diarrhea, leucorrhoea, } \\
\text { menstrual problems }\end{array}$ & Decoction & Aye et al. (2019) \\
\hline & & & Wound healing and diuretic & Decoction & Thongnest et al. (2013) \\
\hline \multirow[t]{3}{*}{ Eriosema cordatum E.Mey } & Roots & South Africa & $\begin{array}{l}\text { Male infertility, erectile dysfunction, } \\
\text { impotence } \\
\text { Aphrodisiac mixed with Corchorus } \\
\text { asplenifolius Burch. }\end{array}$ & $\begin{array}{l}\text { Hot milk infusion; } \\
\text { decoction }\end{array}$ & $\begin{array}{l}\text { Drewes et al. (2002), Drewes et al. } \\
\text { (2013) } \\
\text { Hutchings et al. (1996) }\end{array}$ \\
\hline & & & Female infertility, impotence & Not specified & Bryant (1966) \\
\hline & Leaves & $\begin{array}{l}\text { Republic of } \\
\text { Congo }\end{array}$ & Eye diseases & $\begin{array}{l}\text { Eye instillation of fresh } \\
\text { leaves' juice }\end{array}$ & Kimpouni et al. (2018) \\
\hline $\begin{array}{l}\text { Eriosema crinitum (Kunth) } \\
\text { G. Don }\end{array}$ & Roots & $\begin{array}{l}\text { Brazilia (Cerrado } \\
\text { savannahs) }\end{array}$ & Abortive, contraceptive & Decoction & Rodrigues (2007) \\
\hline $\begin{array}{l}\text { Eriosema diffusum (Kunth) } \\
\text { G. Don }\end{array}$ & Whole plant & $\begin{array}{l}\text { Mexico } \\
\text { Guatemala }\end{array}$ & Unspecified female diseases & Not specified & Hastings (1990) \\
\hline Eriosema englerianum Harms & Roots & Zimbabwe & Bilharziosis & Not specified & Mmbengwa et al. (2009) \\
\hline Eriosema ellipticifolium Schinz & Fruit & South Africa & Dietary supplement & Consumption of fruits & Mabogo (1990) \\
\hline \multirow[t]{8}{*}{$\begin{array}{l}\text { Eriosema glomeratum (Guill. \& } \\
\text { Perr.) Hook.f }\end{array}$} & $\begin{array}{l}\text { Leaves } \\
\text { (petiole) }\end{array}$ & $\begin{array}{l}\text { Gabon (Ngounie } \\
\text { province) }\end{array}$ & Syncope, fish-poison & Decoction & Akendengué and Louis (1994) \\
\hline & & $\begin{array}{l}\text { Benin (Sudano- } \\
\text { Guinean zone) }\end{array}$ & Diabetes & Maceration & Lawin et al. (2015) \\
\hline & Leaves & $\begin{array}{l}\text { Angola (Bakongo } \\
\text { tribes) }\end{array}$ & $\begin{array}{l}\text { Diarrhea, dysentery, cholera, } \\
\text { shigellosis }\end{array}$ & $\begin{array}{l}\text { Consumption of fresh } \\
\text { leaves }\end{array}$ & Göhre et al. (2016) \\
\hline & & $\begin{array}{l}\text { Angola (province } \\
\text { of Uíge) }\end{array}$ & Food/vegetables & $\begin{array}{l}\text { Consumption of fresh } \\
\text { leaves, tea }\end{array}$ & Lautenschläger et al. (2018) \\
\hline & & & Vertigo fainting, syncope & Maceration (bath) & \\
\hline & Leafy stem & Benin & Delayed closure of fontanel & Decoction & Adomou et al. (2012) \\
\hline & Roots & Burkina Faso & Hernia & $\begin{array}{l}\text { Dried, pounded roots } \\
\text { in coffee or porridge }\end{array}$ & Ouoba et al. (2006) \\
\hline & Whole plant & $\begin{array}{l}\text { Cameroon } \\
\text { (Bakola Pygmy } \\
\text { tribe) }\end{array}$ & $\begin{array}{l}\text { Pulmonary troubles, mucosal } \\
\text { infections, nasopharyngeal } \\
\text { infections, leprosy, skin infections, } \\
\text { venereal diseases }\end{array}$ & Decoction & Verdcourt (1970) \\
\hline Eriosema griseum Baker & Leaves & Ivory Coast & $\begin{array}{l}\text { Parasitic diseases associated with } \\
\text { stomach ache, dysentery, diarrhea } \\
\text { in children }\end{array}$ & Not specified & Koné et al. (2005), Koné et al. (2012) \\
\hline \multirow[t]{2}{*}{ Eriosema kraussianum Meisn. } & Roots & South Africa & $\begin{array}{l}\text { Erectile dysfunction, impotence, } \\
\text { urinary complaints in males }\end{array}$ & $\begin{array}{l}\text { Hot milk infusion of } \\
\text { roots; maceration of } \\
\text { root bark }\end{array}$ & $\begin{array}{l}\text { Drewes et al. (2002), Drewes et al. } \\
\text { (2004) }\end{array}$ \\
\hline & & & & & $\begin{array}{l}\text { Bryant (1966); Hutchings et al. (1996) } \\
\text { (Continued on following page) }\end{array}$ \\
\hline
\end{tabular}


TABLE 1 | (Continued) Ethnomedicinal indications and local uses of Eriosema species based on the literature data review.

\begin{tabular}{|c|c|c|c|c|c|}
\hline Species & Parts used & Location & $\begin{array}{l}\text { Local and } \\
\text { traditional uses }\end{array}$ & Preparation & References \\
\hline \multirow[t]{2}{*}{ Eriosema laurentii De Wild. } & Leaves & West Africa & $\begin{array}{l}\text { Food/vegetable, nervous } \\
\text { disorders, laxative, leprosy, } \\
\text { nasopharyngeal affections, } \\
\text { pulmonary problems, skin } \\
\text { diseases, mucosal infections, } \\
\text { venereal diseases, fish-poison }\end{array}$ & Not specified & Burkill (1985) \\
\hline & $\begin{array}{l}\text { Leaves, } \\
\text { roots }\end{array}$ & Cameroon & $\begin{array}{l}\text { Infertility, gynecological and } \\
\text { menopausal complaints }\end{array}$ & Decoction & Ateba et al. (2013b) \\
\hline $\begin{array}{l}\text { Eriosema lebrunii Staner \& De } \\
\text { Craene }\end{array}$ & Leaves & Burundi & $\begin{array}{l}\text { Skin diseases, eczema, impetigo, } \\
\text { dermatoses, ringworm, fungal } \\
\text { infections, athlete's foot, urticaria }\end{array}$ & $\begin{array}{l}\text { Application of juice of } \\
\text { fresh leaves on the } \\
\text { infection site }\end{array}$ & Ngezahayo et al. (2015) \\
\hline \multirow[t]{13}{*}{ Eriosema montanum Bak. f. } & $\begin{array}{l}\text { Not } \\
\text { specified }\end{array}$ & $\begin{array}{l}\text { Democratic } \\
\text { Republic of } \\
\text { Congo }\end{array}$ & $\begin{array}{l}\text { Wound healing, antimicrobial, } \\
\text { disinfectant }\end{array}$ & Not specified & Kasonia et al. (1991) \\
\hline & Leaves & Burundi & Injuries & $\begin{array}{l}\text { Application of } \\
\text { pounded fresh or dry } \\
\text { leaves on the injury }\end{array}$ & Byavu et al. (2000) \\
\hline & & & Oxytocic during childbirth & Decoction & Lewalle and Rodegem (1968) \\
\hline & & & $\begin{array}{l}\text { Impotence, anemia, vitamin } \\
\text { deficiency, delayed motor } \\
\text { development, virility, metrorrhagia, } \\
\text { overwork, tonic }\end{array}$ & $\begin{array}{l}\text { Decoction, } \\
\text { maceration, enema }\end{array}$ & $\begin{array}{l}\text { Polygenis-Bigendako (1990); Baerts } \\
\text { and Lehmann (1989) }\end{array}$ \\
\hline & & & $\begin{array}{l}\text { Otitis, mumps, deafness, earache, } \\
\text { otorrhea }\end{array}$ & $\begin{array}{l}\text { Ear instillation of fresh } \\
\text { leaves' juice }\end{array}$ & $\begin{array}{l}\text { Polygenis-Bigendako (1990); Van } \\
\text { Puyvelde et al. (1977) }\end{array}$ \\
\hline & & & $\begin{array}{l}\text { Snake bite, sting of poisonous } \\
\text { animals }\end{array}$ & $\begin{array}{l}\text { Application of } \\
\text { pounded leaves at the } \\
\text { bite site, decoction }\end{array}$ & $\begin{array}{l}\text { Polygenis-Bigendako (1990); Fumba } \\
\text { (1983); Van Puyvelde et al. (1977); } \\
\text { Baerts and Lehmann (1989) }\end{array}$ \\
\hline & & & $\begin{array}{l}\text { Skin diseases (ringworm, mycosis, } \\
\text { dermatosis, eczema) }\end{array}$ & $\begin{array}{l}\text { Consumption of } \\
\text { calcined dried leaves }\end{array}$ & Ngezahayo et al. (2015) \\
\hline & & & Diarrhea, dysentery, cholera & Decoction, enema & $\begin{array}{l}\text { Ngezahayo et al. (2015); Baerts and } \\
\text { Lehmann (1989) }\end{array}$ \\
\hline & & & $\begin{array}{l}\text { Joint pain, inflammation, } \\
\text { rheumatism }\end{array}$ & $\begin{array}{l}\text { Macerate; vigorous } \\
\text { local rubbing of leaves }\end{array}$ & Van Puyvelde et al. (1977) \\
\hline & & & Eye diseases & $\begin{array}{l}\text { Eye instillation of fresh } \\
\text { leaves' juice }\end{array}$ & Baerts and Lehmann (1989) \\
\hline & & & $\begin{array}{l}\text { Sprain, hematoma, strain, } \\
\text { dislocation, contusion, fracture } \\
\text { Palpitation, heart pain } \\
\text { Pulmonary problems, cough, fever, } \\
\text { vomiting, nausea, anxiety, epilepsy, } \\
\text { depression, nervous disorders, } \\
\text { mental illnesses, analgesic }\end{array}$ & $\begin{array}{l}\text { Local application of } \\
\text { pounded fresh leaves } \\
\text { Not specified } \\
\text { Decoction, steam } \\
\text { bath }\end{array}$ & \\
\hline & & Rwanda & Snake-bites, cough, conjunctivitis & Not specified & $\begin{array}{l}\text { Baerts and Lehmann (1989); } \\
\text { Rwangabo (1993) }\end{array}$ \\
\hline & Roots & Rwanda & Pulmonary problems, cough & $\begin{array}{l}\text { Consumption of raw } \\
\text { roots }\end{array}$ & Durand (1960) \\
\hline Eriosema parviflorum E.Mey & $\begin{array}{l}\text { Leaves, } \\
\text { bark, roots }\end{array}$ & $\begin{array}{l}\text { Tanzania (Kagera } \\
\text { and Lindi regions) }\end{array}$ & Malaria & $\begin{array}{l}\text { Decoction, juice of } \\
\text { fresh leaves }\end{array}$ & $\begin{array}{l}\text { Nondo et al. (2015); Moshi et al. } \\
\text { (2010) }\end{array}$ \\
\hline \multirow[t]{6}{*}{$\begin{array}{l}\text { Eriosema psoraleoides (Lam.) } \\
\text { G.Don }\end{array}$} & Twigs & $\begin{array}{l}\text { Tanzania } \\
\text { (Morogoro) }\end{array}$ & Tooth brush & Not specified & Khan et al. (2000) \\
\hline & Stem bark & $\begin{array}{l}\text { Democratic } \\
\text { Republic of } \\
\text { Congo (Kinshasa) }\end{array}$ & Tuberculosis & Decoction & Ngbolua et al. (2014) \\
\hline & & $\begin{array}{l}\text { Central African } \\
\text { Republic } \\
\text { Republic of } \\
\text { Congo }\end{array}$ & Laxative & Not specified & Sandberg (1965) \\
\hline & Leaves & $\begin{array}{l}\text { Tanzania (Bukoba } \\
\text { rural district) }\end{array}$ & $\begin{array}{l}\text { Health problems related to HIV/ } \\
\text { AIDS such as chronic diarrhea }\end{array}$ & Not specified & Kisangau et al. (2007) \\
\hline & & Central Africa & Oxytocic during childbirth & Water extract & Sillans (1953) \\
\hline & & West Africa & Ectoparasites & Rubbing locally & Dalziel (1937) \\
\hline
\end{tabular}

(Continued on following page) 
TABLE 1 | (Continued) Ethnomedicinal indications and local uses of Eriosema species based on the literature data review.

\begin{tabular}{|c|c|c|c|c|c|}
\hline Species & Parts used & Location & $\begin{array}{l}\text { Local and } \\
\text { traditional uses }\end{array}$ & Preparation & References \\
\hline & & $\begin{array}{l}\text { Central African } \\
\text { Republic }\end{array}$ & $\begin{array}{l}\text { Endoparasites (cestodes, } \\
\text { nematodes, tapeworms, } \\
\text { amoebiasis) }\end{array}$ & Decoction & Wome (1985), Sillans (1953) \\
\hline & & & Diarrhea, dysentery & Maceration & Haxaire (1979) \\
\hline & & & Vaginal prolapse, wound healing & Fumigation & Haxaire (1979), Descoings (1963) \\
\hline & & & Eye diseases & Not specified & Descoings (1963) \\
\hline & & Cameroon & Eye diseases & Maceration (eyewash) & Malzy (1954) \\
\hline & & $\begin{array}{l}\text { Democratic } \\
\text { Republic of } \\
\text { Congo }\end{array}$ & Expectorant & Decoction & Goossens (1924) \\
\hline & & & Fish-poison & Not specified & Goossens (1924) \\
\hline & & & Eye diseases & $\begin{array}{l}\text { Eye instillation of fresh } \\
\text { leaves' juice }\end{array}$ & Wome (1985) \\
\hline & & & Wound healing & $\begin{array}{l}\text { Application of } \\
\text { pounded dried leaves } \\
\text { on the wound }\end{array}$ & Nyakabwa and Gapusi (1990) \\
\hline & & & Abscess, boil, ulcer, acne & $\begin{array}{l}\text { Local application of } \\
\text { pounded fresh leaves }\end{array}$ & Nyakabwa and Gapusi (1990) \\
\hline & & & $\begin{array}{l}\text { Sexually transmited diseases, } \\
\text { expectorant }\end{array}$ & Decoction & Staner and Boutique (1937) \\
\hline & & $\begin{array}{l}\text { Ivory Coast, } \\
\text { Burkina Faso }\end{array}$ & Eye diseases & $\begin{array}{l}\text { Eye instillation of fresh } \\
\text { leaves' juice }\end{array}$ & Kerharo and Bouquet (1950) \\
\hline & & Benin & Skin diseases & $\begin{array}{l}\text { Local application of } \\
\text { fresh leaves' juice }\end{array}$ & Verger (1995) \\
\hline & & Nigeria & Skin diseases, fever, malaria & Decoction & Adjanohoun et al. (1991) \\
\hline & & Rwanda & $\begin{array}{l}\text { Laxative, placental expulsion, } \\
\text { pulmonary problems, cough }\end{array}$ & Decoction & Desouter (1991) \\
\hline & & Burundi & Tonic & Leaf juice & Baerts and Lehmann (1989) \\
\hline & Roots & Tanganyika & $\begin{array}{l}\text { Miscarriage in combination with } \\
\text { Piliostigma trionningii }\end{array}$ & Water extract & Haerdi (1964) \\
\hline & & $\begin{array}{l}\text { Central African } \\
\text { Republic }\end{array}$ & $\begin{array}{l}\text { Otitis, mumps, deafness, earache, } \\
\text { otorrhea }\end{array}$ & $\begin{array}{l}\text { Ear instillation of fresh } \\
\text { leaves' juice }\end{array}$ & Boulesteix et al. (1979) \\
\hline & & & Wound healing & $\begin{array}{l}\text { Application of } \\
\text { scraped root on the } \\
\text { wound }\end{array}$ & Wome (1985) \\
\hline & & $\begin{array}{l}\text { Democratic } \\
\text { Republic of } \\
\text { Congo }\end{array}$ & Sexually transmited diseases & Maceration & Staner and Boutique (1937) \\
\hline & & Uganda & $\begin{array}{l}\text { Stomach ache, gastritis, colic, } \\
\text { colitis, stomach ulcer }\end{array}$ & Decoction & Adjanohoun (1993) \\
\hline & & Rwanda & Pulmonary problems & Consumption of roots & Durand (1960) \\
\hline & & Burundi & Sexually transmited diseases & Decoction & Baerts and Lehmann (1989) \\
\hline & $\begin{array}{l}\text { Roots, } \\
\text { leaves }\end{array}$ & Kenya & $\begin{array}{l}\text { Abdominal pain, gastritis, stomach } \\
\text { aches }\end{array}$ & Decoction & Masinde (1996) \\
\hline & & Tanzania & $\begin{array}{l}\text { Febrile convulsions, fever, malaria, } \\
\text { impotence, frigidity, erectile } \\
\text { dysfunction }\end{array}$ & Decoction & $\begin{array}{l}\text { Moshi et al. (2009), Moshi et al. } \\
\text { (2010) }\end{array}$ \\
\hline & & & $\begin{array}{l}\text { Urogenital infections, miscarriage, } \\
\text { ovarian cyst, pelvic pain }\end{array}$ & Not specified & Haerdi (1964) \\
\hline & Whole plant & Burundi & Diarrhea, dysentery, cholera & Decoction & Polygenis-Bigendako (1990) \\
\hline & Leafy stem & Benin & Jaundice, liver failure, cirrhosis & Not specified & Adjanohoun et al. (1989) \\
\hline & & Togo & Dysmenorrhea, hypermenorrhea & Decoction & Adjanohoun et al. (1986) \\
\hline & $\begin{array}{l}\text { Not } \\
\text { specified }\end{array}$ & $\begin{array}{l}\text { Uganda (Sango } \\
\text { Bay forest } \\
\text { reserve) }\end{array}$ & Malaria & Not specified & Galabuzi et al. (2016) \\
\hline & & $\begin{array}{l}\text { Republic of } \\
\text { Congo }\end{array}$ & Endoparasites & Not specified & Bouquet (1969) \\
\hline $\begin{array}{l}\text { Eriosema pulchellum (Kunth) } \\
\text { G.Don }\end{array}$ & Roots & $\begin{array}{l}\text { Central African } \\
\text { Republic }\end{array}$ & Infertility, impotence & Decoction & Vergiat (1969) \\
\hline Eriosema robustum Baker & $\begin{array}{l}\text { Not } \\
\text { specified }\end{array}$ & East Africa & Coughs & Not specified & Kokwaro (2009) \\
\hline & & Cameroon & Skin diseases & Not specified & Awouafack et al. (2013a) \\
\hline Eriosema rufum (Kunth) G.Don & Roots & Venezuela & Women's sterility, parturition & Not specified & $\begin{array}{l}\text { Morton (1981) } \\
\quad \text { (Continued on following page) }\end{array}$ \\
\hline
\end{tabular}


TABLE 1 | (Continued) Ethnomedicinal indications and local uses of Eriosema species based on the literature data review.

\begin{tabular}{|c|c|c|c|c|c|}
\hline Species & Parts used & Location & $\begin{array}{l}\text { Local and } \\
\text { traditional uses }\end{array}$ & Preparation & References \\
\hline Eriosema salignum E.Mey & $\begin{array}{l}\text { Roots, } \\
\text { rootbark }\end{array}$ & South Africa & $\begin{array}{l}\text { Male sexual disorders, erectile } \\
\text { dysfunction, impotence }\end{array}$ & $\begin{array}{l}\text { Hot milk infusion of } \\
\text { roots, maceration of } \\
\text { root bark }\end{array}$ & $\begin{array}{l}\text { Drewes et al. (2002), Drewes et al. } \\
\text { (2013) } \\
\text { Hulme (1954) }\end{array}$ \\
\hline Eriosema scioanum Avetta & Leaves & Ethiopia & Sexually transmited diseases & Not specified & Lemordant (1972) \\
\hline Eriosema stanerianum Hauman & Leaves & $\begin{array}{l}\text { Uganda (Sango } \\
\text { Bay area) }\end{array}$ & Malaria & Decoction & Ssegawa and Kasenene (2007) \\
\hline $\begin{array}{l}\text { Eriosema tisserantii Staner \& De } \\
\text { Craene }\end{array}$ & Roots & $\begin{array}{l}\text { Central Africa } \\
\text { Central African } \\
\text { Republic }\end{array}$ & $\begin{array}{l}\text { Aphrodisiac, erectile dysfunction } \\
\text { Women's sterility, frigidity }\end{array}$ & $\begin{array}{l}\text { Water extract } \\
\text { Decoction }\end{array}$ & $\begin{array}{l}\text { Sillans (1953) } \\
\text { Vergiat (1969) }\end{array}$ \\
\hline
\end{tabular}

Eriosema robustum Baker is widespread in Burundi, Ethiopia, Kenya, Rwanda, Tanzania, Uganda, Democratic Republic of Congo and Cameroon (Gillett et al., 1971). It is believed to cure coughs in East Africa (Kokwaro, 2009) and skin diseases in Central Africa (Awouafack et al., 2013a).

Eriosema laurentii De Wild. widely dispersed in West and Central Africa is used in this area as food and herbal medicine for many purposes such as nasopharyngeal, brain and nervous system affections, pulmonary troubles and venereal diseases (Burkill, 1985). In Cameroon, the plant is utilized for the treatment of infertility and various gynecological problems (Ateba et al., 2013).

The roots of Eriosema chinense Vogel (syn. Eriosema himalaicum H. Ohashi; Eriosema tuberosum (Ham.) Wang et Tang) are reported to be used as food in Northern Australia, China and North East India and against diarrhea in Meghalaya (India) (Neogi et al., 1989; Martin and Ryan, 2004; Prasad et al., 2013a). In Yunnan Province of China, the roots are used against diarrhea, orchitis, hydrophobia and as detoxifying medicine (Kunming Institute of Botany, 1979). In Thailand, the fresh roots are eaten as a tonic, and decoctions of the seeds are used for wound healing and for their diuretic properties (Thongnest et al., 2013). Such decoctions are also well-known as astringent, diuretic, tonic, against cold sweats and during delivery to promote discharge of the lochia. Decoctions additionally containing powdered pepper are given for diarrhea (Ashraf and Borthakur, 2005; Laloo and Hemalatha, 2011; Prasad et al., 2013c; Thongnest et al., 2013).

Eriosema psoraleoides (Lam.) G. Don. is a species widely used over Africa from Togo to Tanzania. In Tanzania, peeled or unpeeled twigs are used as chewing sticks (Khan et al., 2000), while leaves are used against health problems related to HIV/ AIDS such as chronic diarrhea (Kisangau et al., 2007), impotence, erectile dysfunction, fever and malaria (Moshi et al., 2009, 2010). In several countries in Central and West Africa, leaves are applied against eye diseases (Kerharo and Bouquet, 1950; Malzy, 1954; Descoings, 1963; Wome, 1985). The roots are used in Central African Republic against ear problems (Boulesteix and Guinko, 1979), and in Democratic Republic of Congo and Burundi against sexually transmitted diseases (Staner and Boutique, 1937; Baerts and Lehmann, 1989). Eating raw roots is claimed to treat pulmonary problems in Rwanda (Durand, 1960).

Leaves of Eriosema griseum Baker are known as infant cures for treating parasitic diseases and associated discomforts such as stomach ache, dysentery or diarrhea in Northern parts of Ivory Coast (Koné 2005; Koné et al., 2012).

The roots of Eriosema englerianum Harms are traditionally used in Zimbabwe in combination with other plants such as Vigna ungiculata and Terminalia sericea to treat bilharziosis (Mmbengwa et al., 2009).

In southeastern Brazil (Cerrado of Minas Gerais), Eriosema glabrum Mart. ex Benth. is a traditional laxative, while Eriosema benthamianum Mart. ex Benth. and Eriosema campestre var. macrophyllum (Gear) Fortunato are reported as antiinflammatory agents (Hirschmann and De Arias, 1990).

Globally, the search of information resulted in 25 species $(\sim 16.6 \%$ of the genus), for which written evidence of traditional uses is available. Clearly, this list cannot be exhaustive as in many traditional medicine systems a vast knowledge on medicinal plants exists as oral information only. The majority of medicinal plants used over the world are harvested from wild resources in increasing volumes. Given their integral role in basic healthcare in many developing countries, their conservation and sustainable use are a necessity. The information of the IUCN (International Union for Conservation of Nature) Red List Categories, assessing the conservation status of species, was accessed for Eriosema species from https://www.iucnredlist.org/fr/ search?query=Eriosema\&searchType=species. At the moment, only 22 Eriosema species (Table 2) are included in this list. Information for the vast majority of species widely used in traditional medicine such as E. chinense, E. cordatum, E. glomeratum and E. psoraleoides and others depicted in Table $\mathbf{1}$ is still missing. Only the three traditionally used species Eriosema crinitum (Kunth) G. Don, Eriosema englerianum Harms and Eriosema montanum Baker f. are listed, characterized by a stable population with least concern. Nevertheless, the most popular species with traditional use should be assessed to avoid a negative impact on the size of the populations. Root and whole-plant harvesting is more destructive than collecting leaves and flowers or buds (Chen et al., 2016). Among the 25 species traditionally used and reported in this review, mainly roots are utilized (18 species), followed by leaves (11 species), bark and whole plant (3 species), seeds or fruits (2 species) and twigs (1 species) (Figure 1). Rather to use roots, leaves can be an alternative to avoid the destruction and highspeed disappearing of Eriosema species. In case roots are unavoidable for the use, non-destructive harvest protocols such as partial-root harvest should be applied. 
TABLE 2 | The International Union for Conservation of Nature (IUCN) Red List Categories of Eriosema species.

\begin{tabular}{|c|c|c|c|c|}
\hline Species & Scope of assessment & Population trend & $\begin{array}{c}\text { Date of the last } \\
\text { assessment }\end{array}$ & $\begin{array}{c}\text { IUCN } \\
\text { red list categories }\end{array}$ \\
\hline Eriosema adamaouense Jacq.-Fel. & Global & Decreasing & March 25, 2011 & CR \\
\hline Eriosema adami Jacq.-Fel. & Global & Unknown & December 18, 2018 & $\mathrm{CR}$ \\
\hline Eriosema arachnoideum Verdc. & Global & Unknown & September 03, 2011 & EN \\
\hline Eriosema arenicola (Verdc.) Maesen \& Wieringa & Global & Unknown & December 04, 2018 & VU \\
\hline Eriosema benguellense Rossberg & Global & Unknown & June 30, 2010 & DD \\
\hline Eriosema betsileense Du Puy \& Labat & Global & Decreasing & September 03, 2015 & EN \\
\hline Eriosema crinitum (Kunth) G. Don & Global & Stable & August 13, 2010 & LC \\
\hline Eriosema englerianum Harms & Global & Stable & April 23, 2010 & LC \\
\hline Eriosema harmsiana Dinter & Global & Stable & April 30, 2004 & LC \\
\hline Eriosema latericola Jacq.-Fel. & Global & Unknown & December 19, 2018 & EN \\
\hline Eriosema letouzeyi Jacq.-Fel. & Global & Decreasing & March 25, 2011 & VU \\
\hline Eriosema longicalyx Grear & Global & Stable & September 16, 2010 & LC \\
\hline Eriosema montanum Baker f. & Global & Stable & September 03, 2011 & LC \\
\hline Eriosema pauciflorum Klotzsch & Global & Unknown & July 06, 2016 & LC \\
\hline Eriosema procumbens Baker & Global & Unknown & September 01, 2015 & LC \\
\hline Eriosema pseudodistinctum Verdc. & Global & Unknown & September 03, 2011 & EN \\
\hline Eriosema pseudostolzii Verdc. & Global & Unknown & September 03, 2011 & VU \\
\hline Eriosema raynaliorum Jacq.-Fel. & Global & Unknown & July 14, 2015 & LC \\
\hline Eriosema spicatum Hook.f & Global & Unknown & February 18, 2019 & LC \\
\hline Eriosema triformum Burgt & Global & Decreasing & November 11, 2016 & $\mathrm{CR}$ \\
\hline Eriosema violaceum (Aubl.) G. Don & Global & Stable & June 12, 2018 & LC \\
\hline Eriosema chevalieri (Harms) Hutch. \& Dalziel (syn. Rhynchosia chevalieri Harms) & Global & Unknown & January 07, 2019 & EN \\
\hline
\end{tabular}

$C R$, critically endangered; $D D$, data deficient; EN, endangered; LC, least concern; VU, vulnerable.

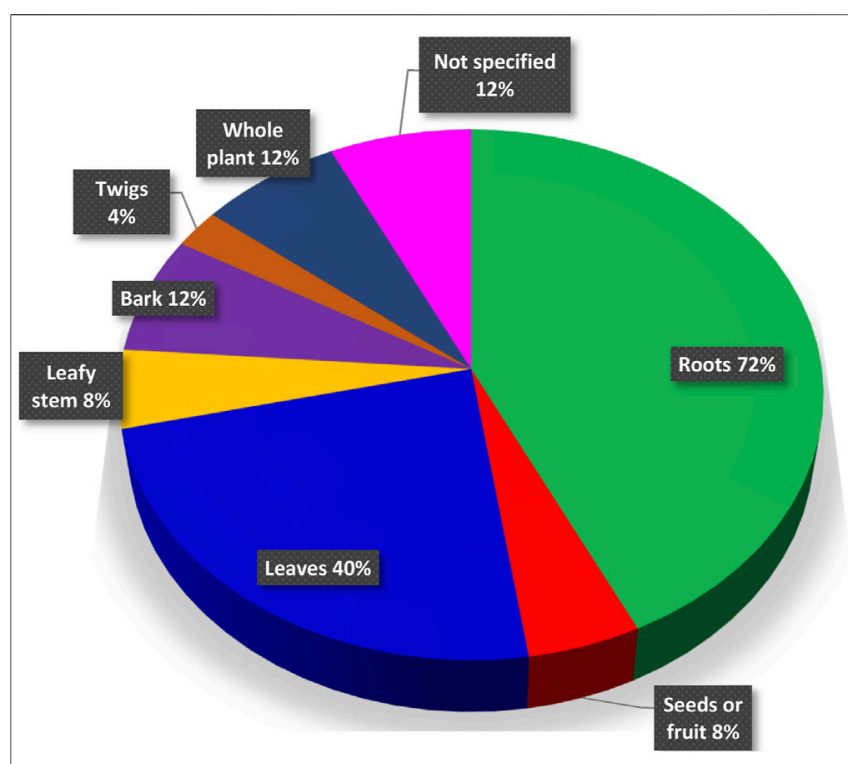

FIGURE 1 | Traditionally used plant parts of Eriosema species.

\section{PHYTOCHEMISTRY OF THE GENUS}

Based on the ethnopharmacological applications, numerous chemical analyses of Eriosema species have been carried out. Compounds identified or isolated are depicted in Table 3 and Figure 2.

\section{Flavonoids}

In their review, Awouafack et al. (2015) compiled a total of 52 natural flavonoids (indicated by $\left(^{*}\right)$ in Table 3 ) from five Eriosema species (E. chinense, E. tuberosum, E. kraussianum, E. glomeratum and E. robustum), including dihydrochalcones (1-2), flavonols (10-13, 16-20), flavanonols or dihydroflavonols (23-33), flavanones (34-41), isoflavones $(43,47-52,54,56-60$, 62, 64-70) and one isoflavanone (71). Flavonoids/isoflavonoids not recorded in that review and/or from different Eriosema species are mentioned below.

In a dereplication approach, the flavones isovitexin (3), luteolin (6), isoorientin (7) and luteolin-7-O-glucoside (8) were unambiguously identified in a methanol extract of the aerial parts of E. laurentii (Ateba et al., 2014b) and 2"-Oa-L-rhamnosyl-6-C-fucosyl-3'-O-methyl-luteolin (9) was isolated from the same extract (Ateba et al., 2016a). Vitexin (4) and robusflavone C (5) were extracted from an ethanol fraction of twigs of E. robustum (Awouafack et al., 2018).

The flavonol kaempferol (10) was isolated from roots of $E$. chinense (Thongnest et al., 2013) and twigs of E. robustum (Awouafack et al., 2018).

Quercetin (13) and quercetin-3-O-methylether (14) were identified in a methanol extract of the aerial parts of $E$. laurentii (Ateba et al., 2014b), while 3,4',6,8-tetrahydroxyflavone-7$C$-glucoside (15) from that extract was identified for the first time in the genus (Ateba et al., 2016a).

The flavanonols 3,4',6,8-tetrahydroxyflavanone-7-C-glucoside (21) and (2S,3S)-6,8,3'-triprenyl-dihydromorin (22) from the methanol extracts of the aerial (Ateba et al., 2016a) and underground (Ateba et al., 2016b) parts of E. laurentii, 
TABLE 3 | Compounds isolated/identified from Eriosema species (the structure of compounds illustrated in Figure 2).

\begin{tabular}{llcc}
\hline Chemical classes & $\mathbf{N}^{\circ}$ & Compounds & Plant parts \\
and species & References
\end{tabular}

\begin{tabular}{|c|c|c|c|c|}
\hline \multicolumn{5}{|l|}{ Flavonoids } \\
\hline \multirow[t]{2}{*}{ Dihydrochalcones } & 1. & Erioschalcone a or $2^{\prime}, 4^{\prime}$-dihydroxy-4-methoxy-3'-( $\gamma, \gamma$-dimethylallyl) dihydrochalcone $\left(^{*}\right)$ & Whole plant of $E$. glomeratum & Awouafack et al. (2008) \\
\hline & 2. & Erioschalcone B or 2',4'-dihydroxy-3'-( $\gamma, \gamma$-dimethylallyl)dihydrochalcone $\left(^{*}\right)$ & Whole plant of E. glomeratum & Awouafack et al. (2008) \\
\hline \multirow{7}{*}{ Flavones } & 3. & Isovitexin or Apigenin-6-C-glucoside & Aerial parts of E.laurentii & Ateba et al. (2014b) \\
\hline & 4. & Vitexin & Twigs of $E$. robustum & Awouafack et al. (2018) \\
\hline & 5. & Robusflavone C & Twigs of $E$. robustum & Awouafack et al. (2018) \\
\hline & 6. & Luteolin & Aerial parts of $E$. laurentii & Ateba et al. (2014b) \\
\hline & 7. & Isoorientin or Luteolin-6-C-glucoside & Aerial parts of $E$. laurentii & Ateba et al. (2014b) \\
\hline & 8. & Luteolin-7-O-glucoside & Aerial parts of $E$. laurentii & Ateba et al. (2014b) \\
\hline & 9. & 2"-O-a-L-Rhamnosyl-6-C-fucosyl-3'-O-methyl-luteolin & Aerial parts of $E$. laurentii & Ateba et al. (2016a) \\
\hline \multirow[t]{11}{*}{ Flavonols } & 10. & Kaempferol $\left({ }^{\star}\right)$ & Roots of $E$. chinense & Thongnest et al. (2013) \\
\hline & 11. & Kaempferol-7-O- $\beta$-D-glucopyranoside (*) & Roots of $E$. chinense & Thongnest et al. (2013) \\
\hline & 12. & Astragalin or Kaempferol-3-O- $\beta$-D-glucopyranoside $\left(^{*}\right)$ & Roots of $E$. chinense & Thongnest et al. (2013) \\
\hline & 13. & Quercetin $\left(^{*}\right)$ & $\begin{array}{l}\text { Whole plant of } E \text {. glomeratum } \\
\text { Aerial parts of } E \text {. laurentii }\end{array}$ & $\begin{array}{l}\text { Awouafack et al. (2008) } \\
\text { Ateba et al. (2014b) }\end{array}$ \\
\hline & 14. & Quercetin-3-O-methylether & Aerial parts of $E$. laurentii & Ateba et al. (2014b) \\
\hline & 15. & 3,4',6,8-Tetrahydroxyflavone-7-C-glucoside & Aerial parts of $E$. laurentii & Ateba et al. (2016a) \\
\hline & 16. & Robusflavone A or $2^{\prime}, 3^{\prime}, 5^{\prime}, 5,7$-pentahydroxy-3,4'-dimethoxyflavone $\left({ }^{*}\right)$ & Twigs of $E$. robustum & Awouafack et al. (2013a) \\
\hline & 17. & Robusflavone B or $2^{\prime}, 3,5^{\prime}, 5,7$-pentahydroxy-4'-methoxyflavone (*) & Twigs of $E$. robustum & Awouafack et al. (2013a) \\
\hline & 18. & Dehydrolupinifolinol $\left({ }^{*}\right)$ & Roots of E.chinense & Sutthivaiyakit et al. (2009) \\
\hline & 19. & $\begin{array}{l}\text { Khonklonginol F or 3,5-dihydroxy-4'-methoxy-6", } 6^{\prime \prime} \text {-dimethylpyrano }\left(2^{\prime \prime}, 3^{\prime \prime}: 7,6\right)-8-\left(3^{\prime \prime \prime}, 3^{\prime \prime \prime}-\right. \\
\text { dimethylally) flavone }\left(^{(*)}\right.\end{array}$ & Roots of E. chinense & Sutthivaiyakit et al. (2009) \\
\hline & 20. & $3,5,2^{\prime}, 4^{\prime}-$ Tetrahydroxy-6", $6^{\prime \prime}-$ dimethylpyrano $\left(2^{\prime \prime}, 3^{\prime \prime}: 7,6\right)-8-\left(3^{\prime \prime \prime}, 3^{\prime \prime \prime}-\right.$ dimethylally)flavone (*) & Roots of $E$. chinense & Thongnest et al. (2013) \\
\hline \multirow{6}{*}{$\begin{array}{l}\text { Flavanonols or } \\
\text { dihydroflavonols }\end{array}$} & 21. & 3,4',6,8-Tetrahydroxyflavone-7-C-glucoside & Aerial parts of $E$. laurentii & Ateba et al. (2016a) \\
\hline & 22. & $\begin{array}{l}(2 S, 3 S)-6,8,3^{\prime} \text {-Triprenyl-dihydromorin or }(2 S, 3 S)-6,8,3^{\prime} \text {-triprenyl-3,5,7,2', } 4^{\prime} \text { - } \\
\text { pentahydroxyflavanone }\end{array}$ & Underground parts of $E$. laurentii & Ateba et al. (2016b) \\
\hline & 23. & Lupinifolinol $\left({ }^{*}\right)$ & Roots of $E$. chinense & $\begin{array}{l}\text { Sutthivaiyakit et al. (2009), Thongnest } \\
\text { et al. (2013) }\end{array}$ \\
\hline & 24. & $\begin{array}{l}\text { 3-epi-Lupinifolinol or }(2 R, 3 S)-3,5,4^{\prime} \text {-trihydroxy-6", } 6^{\prime \prime} \text { dimethylpyrano }\left(2^{\prime \prime}, 3^{\prime \prime}: 7,6\right)-8-\left(3^{\prime \prime \prime}, 3^{\prime \prime \prime}-\right. \\
\text { dimethylallyl) flavanone }\left({ }^{*}\right)\end{array}$ & Roots of $E$. chinense & Thongnest et al. (2013) \\
\hline & 25. & $\begin{array}{l}\text { 2'-Hydroxylupinifolinol or }(2 R, 3 R)-3,5,2^{\prime}, 4^{\prime} \text {-tetrahydroxy-6", } 6^{\prime \prime} \text {-dimethylpyrano }\left(2^{\prime \prime}, 3^{\prime \prime}: 7,6\right) \text { - } \\
8-\left(3^{\prime \prime \prime}, 3^{\prime \prime \prime} \text {-dimethylally)fflavanone }\left(^{*}\right)\right.\end{array}$ & Roots of $E$. chinense & Thongnest et al. (2013) \\
\hline & 26. & $\begin{array}{l}\text { 3-epi-Khonklonginol C or }(2 R, 3 S)-3,5,2^{\prime} \text {-trihydroxy-4'-methoxy-6", } 6^{\prime \prime} \text {-dimethylpyrano } \\
\left(2^{\prime \prime}, 3^{\prime \prime}: 7,6\right)-8-\left(3^{\prime \prime \prime}, 3^{\prime \prime \prime} \text {-dimethylallyl) flavanone }\left(^{*}\right)\right.\end{array}$ & Roots of $E$. chinense & Thongnest et al. (2013) \\
\hline
\end{tabular}


TABLE 3 | (Continued) Compounds isolated/identified from Eriosema species (the structure of compounds illustrated in Figure 2).

\begin{tabular}{|c|c|c|c|c|}
\hline Chemical classes & $\mathbf{N}^{\circ}$ & Compounds & $\begin{array}{l}\text { Plant parts } \\
\text { and species }\end{array}$ & References \\
\hline & 27. & $\begin{array}{l}\text { Khonklonginol A or 3,5-dihydroxy-4'-methoxy-6", } 6^{\prime \prime} \text {-dimethylpyrano }\left(2^{\prime \prime}, 3^{\prime \prime}: 7,6\right)-8-\left(3^{\prime \prime \prime}, 3^{\prime \prime \prime}-\right. \\
\text { dimethylallyl) flavanone }\left(^{\star}\right)\end{array}$ & Roots of E. chinense & Thongnest et al. (2013) \\
\hline & & & & Sutthivaiyakit et al. (2009) \\
\hline & 28. & $\begin{array}{l}\text { Khonklonginol B or 3,5-dihydroxy-4'-methoxy-6", } 6^{\prime \prime} \text {-dimethylpyrano }\left(2^{\prime \prime}, 3^{\prime \prime}: 7,6\right)-8-\left(3^{\prime \prime \prime}, 3^{\prime \prime \prime}-\right. \\
\text { dimethylallyl) flavanone ( }\left(^{*}\right)\end{array}$ & Roots of $E$. chinense & Sutthivaiyakit et al. (2009) \\
\hline & 29. & $\begin{array}{l}\text { Khonklonginol C or } 3,5,2^{\prime} \text {-trihydroxy-4'-methoxy-6" } 6^{\prime \prime}, 6^{\prime \prime} \text {-dimethylpyrano }\left(2^{\prime \prime}, 3^{\prime \prime}: 7,6\right)-8 \text { - } \\
\left(3^{\prime \prime \prime}, 3^{\prime \prime \prime} \text {-dimethylallyl) flavanone }\left(^{*}\right)\right.\end{array}$ & Roots of $E$. chinense & Sutthivaiyakit et al. (2009) \\
\hline & 30. & $\begin{array}{l}\text { Khonklonginol D or 3,5-dihydroxy-3',4'-dimethoxy-6",6"-dimethylpyrano (2", 3": 7,6)-8- } \\
\left(3^{\prime \prime \prime}, 3^{\prime \prime \prime}-\text {-dimethylally) flavanone }\left(^{*}\right)\right.\end{array}$ & Roots of $E$. chinense & Sutthivaiyakit et al. (2009) \\
\hline & 31. & $\begin{array}{l}\text { Khonklonginol E or 3,5-dihydroxy-4'-methoxy-6", } 6^{\prime \prime} \text {-dimethylpyrano }\left(2^{\prime \prime}, 3^{\prime \prime}: 7,6\right)-8-\left(3^{\prime \prime \prime}, 3^{\prime \prime \prime}-\right. \\
\text { dimethyl-2"', } 3^{\prime \prime \prime} \text {-dihydroxypropyl) flavanone (") }\end{array}$ & Roots of $E$. chinense & Sutthivaiyakit et al. (2009) \\
\hline & 32. & $\begin{array}{l}\left(2 R, 3 R, 2^{\prime \prime \prime} R\right)-3,5,2^{\prime \prime \prime}-\text { Trihydroxy-4'-methoxy-6" } 6^{\prime \prime}, 6^{\prime \prime}-\text { dimethylpyrano }\left(2^{\prime \prime}, 3^{\prime \prime}: 7,6\right)-8-\left(3^{\prime \prime \prime}-\right. \\
\text { methylbut-3"'-enyl) flavanone }\left(^{*}\right)\end{array}$ & Roots of E. chinense & Thongnest et al. (2013) \\
\hline & 33. & 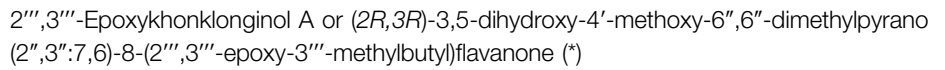 & Roots of $E$. chinense & Thongnest et al. (2013) \\
\hline \multirow[t]{12}{*}{ Flavanones } & 34. & Eriosemaone $\mathrm{A}\left({ }^{*}\right)$ & Roots of E. tuberosum & Ma et al. (1995) \\
\hline & & & Roots of $E$. chinense & Sutthivaiyakit et al. (2009) \\
\hline & 35. & Eriosemaone $\mathrm{B}\left(^{\star}\right)$ & Roots of E. tuberosum & Ma et al. (1995) \\
\hline & 36. & Eriosemaone $C\left(^{*}\right)$ & Roots of E. tuberosum & Ma et al. (1995) \\
\hline & 37. & $\begin{array}{l}\text { Khonklonginol G or 5-hydroxy-4'-methoxy-6", } 6^{\prime \prime} \text {-dimethylpyrano }\left(2^{\prime \prime}, 3^{\prime \prime}: 7,6\right)-8-\left(3^{\prime \prime \prime}, 3^{\prime \prime \prime}-\right. \\
\text { dimethylallyl) flavanone }\left(^{*}\right)\end{array}$ & Roots of $E$. chinense & Sutthivaiyakit et al. (2009) \\
\hline & 38. & $\begin{array}{l}\text { Khonklonginol } \mathrm{H} \text { or } 5,2^{\prime} \text {-dihydroxy-4'-methoxy-6", } 6^{\prime \prime} \text {-dimethylpyrano }\left(2^{\prime \prime}, 3^{\prime \prime}: 7,6\right)-8-\left(3^{\prime \prime \prime}, 3^{\prime \prime \prime}-\right. \\
\text { dimethylallyl) flavanone }\left(^{*}\right)\end{array}$ & Roots of $E$. chinense & Sutthivaiyakit et al. (2009) \\
\hline & 39. & Lupinifolin $\left(^{*}\right)$ & Roots of $E$. tuberosum & Ma et al. (1996b) \\
\hline & & & Roots of $E$. chinense & $\begin{array}{l}\text { Prasad et al. (2013a) } \\
\text { Sutthivaiyakit et al. (2009) }\end{array}$ \\
\hline & 40. & Flemichin $\mathrm{D}\left({ }^{*}\right)$ & Roots of $E$. tuberosum & Ma et al. (2015) \\
\hline & & & Roots of $E$. chinense & $\begin{array}{l}\text { Sutthivaiyakit et al. (2009), } \\
\text { Thongnest et al. (2013) }\end{array}$ \\
\hline & 41. & 6-Prenyl pinocembrin $\left(^{*}\right)$ & Twigs of E. robustum & Awouafack et al. (2013a) \\
\hline & 42. & Prunin & Twigs of E. robustum & $\begin{array}{l}\text { Awouafack et al. (2018) } \\
\text { (Continued on following page) }\end{array}$ \\
\hline
\end{tabular}


TABLE 3 | (Continued) Compounds isolated/identified from Eriosema species (the structure of compounds illustrated in Figure 2).

\begin{tabular}{|c|c|c|}
\hline Chemical classes & $\mathbf{N}^{\circ}$ & Compound \\
\hline \multirow[t]{12}{*}{ Isoflavones } & 43. & Genistein $\left(^{*}\right)$ \\
\hline & 44. & 2'-Hydroxygenistein \\
\hline & 45. & 5,7,4'-Trihdroxy-2'-methoxyisoflavone \\
\hline & 46. & $4^{\prime}, 5$-Dihydroxy-2', 7 -dimethoxyisoflavone \\
\hline & 47. & Isoluteolin $\left(^{\star}\right)$ \\
\hline & 48. & 5-O-Methylgenistein $\left(^{*}\right)$ \\
\hline & 49. & Tectorigenin $\left(^{\star}\right)$ \\
\hline & 50. & 7-O-Methyltectorigenin (*) \\
\hline & 51. & 5,7,2',4'-Tetrahydroxy-6-methoxyisoflavone (*) \\
\hline & 52. & 6,7-Dimethoxy-5,2',4'-trihydroxyisoflavone $\left(^{*}\right)$ \\
\hline & 53. & $4^{\prime}, 7^{\prime \prime}$-Bisgenistein \\
\hline & 54. & Genistin or genistein-7-O- $\beta$-D-glucopyranoside $\left({ }^{*}\right)$ \\
\hline
\end{tabular}

Genistin or genistein-7-O- $\beta$-D-glucopyranoside $\left(^{*}\right.$

\section{Genistein-8-C-glucopyranoside}

5-O-Methylgenistein-7-O- $\beta$-D-glucopyranoside $\left(^{*}\right)$

Eriosemaside $\mathrm{C}$ or 5-O-Methylgenistein-7-O- $\beta$-D-apiofuranosyl-(1 $\rightarrow 2)-O-\beta$-D-

glucopyranoside $\left(^{*}\right)$

Genistein-7-O- $\beta$-D-apiofuranosyl-( $1 \rightarrow 6)$-O- $\beta$-D-glucopyranoside $\left(^{*}\right)$

5-O-Methylgenistein-7-O- $\beta$-D-apiofuranosyl-(1 $\rightarrow 6)$-O- $\beta$-D-glucopyranoside (*)

Sphaerobioside (*)

$2^{\prime}$-Hydroxygenistein-7-O-glucopyranoside

Eriosemaone D or $5,7,2^{\prime}$-trihydroxy-6", $6^{\prime \prime}$-dimethylpyrano $\left(2^{\prime \prime}, 3^{\prime \prime}: 4^{\prime}, 5^{\prime}\right)$ isoflavone $\left(^{*}\right)$

2'-O-Methyl-eriosemaone D or 5,7-dihydroxy-2'-methoxy-6", $6^{\prime \prime}$-dimethyl-pyrano (2", 3": $\left.4^{\prime}, 5^{\prime}\right)$ isoflavone

64. Kraussianone 1 or $5,2^{\prime}$-dihydroxy-[(6", $6^{\prime \prime}$-dimethylpyrano $\left.\left(2^{\prime \prime}, 3^{\prime \prime}: 4^{\prime}, 5^{\prime}\right)\right]\left[\left(6^{\prime \prime \prime}, 6^{\prime \prime \prime}\right.\right.$ dimethylpyrano $\left.\left(2^{\prime \prime \prime}, 3^{\prime \prime \prime}: 7,6\right)\right]$ isoflavone $\left(^{*}\right)$
Plant parts

and specir

\section{References}

Roots and twigs of $E$. tuberosum Ma et al. (1998)

Roots of E. chinense Awouafack et al. (2018)

Aerial and underground parts of Ateba et al. (2013),

E. laurentii Ateba et al. (2016b)

Aerial and underground parts of Ateba et al. (2014b),

E. laurenti

Ateba et al. (2016b)

Roots of E. psoraleoides Wanyama (2010)

Roots of E. psoraleoides Wanyama (2010)

Whole plant of $E$. glomeratum Awouafack et al. (2008)

Roots of $E$ tuberosum Ma et al. (1998)

Roots of E. chinense Thongnest et al. (2013)

Roots of E. chinense Thongnest et al. (2013)

Roots of E. chinense Thongnest et al. (2013)

Roots of $E$. chinense Thongnest et al. (2013)

Roots of E. psoraleoides Wanyama (2010)

Roots of E. tuberosum Ma et al. (1998)

Aerial and underground parts of Ateba et al. (2013),

E. laurentii $\quad$ Ateba et al. (2016b)

Roots of E. chinense Thongnest et al. (2013)

Aerial parts of $E$. laurentii $\quad$ Ateba et al. (2016a)

Roots of $E$. tuberosum Ma et al. (1998)

Roots of E. tuberosum Ma et al. (1999)

Roots of E. tuberosum Ma et al. (1998)

Roots of E. tuberosum Ma et al. (1998)

Roots of E tuberosum

Aerial parts of E. laurentii Ateba et al. (2016a)

Roots of E. tuberosum Ma et al. (1995)

Underground parts of E. laurentii Ateba et al. (2016b)

Underground parts of $E$. laurentii Ateba et al. (2016b)

Rootstock of E. kraussianum

Drewes et al. (2002)

Ojewole et al. (2006)

Drewes et al. (2002)

Ojewole et al. (2006)

Ramesar et al. (2012)

Rootstock of $E$. kraussianum

Rootstock of E. kraussianum

Rootstock of $E$. kraussianum

Rootstock of $E$. kraussianum

Drewes et al. (2002)

Drewes et al. (2002)

Drewes et al. (2002)

Rootstock of $E$. kraussianum

Drewes et 
TABLE 3 | (Continued) Compounds isolated/identified from Eriosema species (the structure of compounds illustrated in Figure 2).

\begin{tabular}{|c|c|c|c|c|}
\hline Chemical classes & $\mathbf{N}^{\circ}$ & Compounds & $\begin{array}{l}\text { Plant parts } \\
\text { and species }\end{array}$ & References \\
\hline Isoflavanones & 71. & Cajanol $\left({ }^{*}\right)$ & Roots of $E$. chinense & Thongnest et al. (2013) \\
\hline \multirow[t]{4}{*}{ Coumaronochromones } & 72. & Eriocoumaronochromone & Twigs of E. robustum & Awouafack et al. (2018) \\
\hline & 73. & Lupinalbin A or 5.7,4'-trihydroxy-coumaronochromone & Aerial and underground parts of & Ateba et al. (2014b), \\
\hline & & & Roots of E. psoraleoides & Wanyama (2010) \\
\hline & 74. & Desmoxyphyllin A or 5,7,4'-trihydroxy-5'-methoxycoumaronochromone & Twigs of $E$. robustum & Awouafack et al. (2018) \\
\hline \multicolumn{5}{|l|}{ Chromones } \\
\hline & 75. & Eriosematin or 5-hydroxy-8- $\gamma, \gamma$-dimethylallyl-6", $6^{\prime \prime}$-dimethylpyrano $\left(3^{\prime \prime}, 2^{\prime \prime}: 6,7\right)$ chromone & Roots of E. tuberosum & Ma et al. (1995) \\
\hline & 76. & $\begin{array}{l}\text { Iso-eriosematin or 5-hydroxy-6- } \gamma, \gamma \text {-dimethylallyl-6',6'-dimethyl-pyrano }\left(2^{\prime}, 3^{\prime}: 7,8\right) \\
\text { chromone }\end{array}$ & Roots of E. tuberosum & Ma et al. (1996a) \\
\hline & 77. & Eriosematin A or 5,7-dihydroxy-8- $\gamma, \gamma$-dimethylallyl chromone & Roots of $E$. tuberosum & Ma et al. (1996a) \\
\hline & 78. & $\begin{array}{l}\text { Eriosematin B or 5,7-dihydroxy-6-(3-hydroxy-3,3-dimethylbutyl)-8- } \gamma, \gamma \text {-dimethylallyl } \\
\text { chromone }\end{array}$ & Roots of $E$. tuberosum & Ma et al. (1996a) \\
\hline & 79. & Eriosematin C or 5,7-dihydroxy-6- $\gamma, \gamma$-dimethylallyl-8- $\gamma, \gamma$-dimethylallyl chromone & Roots of E. tuberosum & Ma et al. (1996a) \\
\hline & 80. & $\begin{array}{l}\text { Eriosematin D or } 2,5 \text {-dihydroxy-6- } \gamma, \gamma \text {-dimethylallyl-6",6"-dimethylpyrano }\left(2^{\prime \prime}, 3^{\prime \prime}: 7,8\right) \\
\text { chromone }\end{array}$ & Roots of $E$. tuberosum & Ma et al. (1996b) \\
\hline & 81. & $\begin{array}{l}\text { Eriosematin E or } 2,5 \text {-dihydroxy-8- } \gamma, \gamma \text {-dimethylallyl-6",6"-dimethylpyrano }\left(2^{\prime \prime}, 3^{\prime \prime}: 7,6\right) \\
\text { chromone }\end{array}$ & Roots of E. tuberosum & Ma et al. (1996b) \\
\hline & & & Roots of $E$. chinense & Prasad et al. (2017) \\
\hline & 82. & Eriosemaside A or 5,7-dihydroxy-8- $\gamma, \gamma$-dimethylallylchromone-7-O-rutinoside & Roots of $E$. tuberosum & Ma et al. (1999) \\
\hline \multicolumn{5}{|l|}{ Lignans } \\
\hline & 83. & Syringaresinol & Aerial parts of $E$. laurentii & Ateba et al. (2016a) \\
\hline & 84. & Yangambin & Roots of $E$. chinense & Sutthivaiyakit et al. (2009) \\
\hline \multicolumn{5}{|l|}{ Terpenoids } \\
\hline Triterpenoid & 85. & Olean-12- ene-3,16,23-triol & Whole plant of E. glomeratum & Awouafack et al. (2008) \\
\hline \multirow[t]{6}{*}{ Sterols } & 86. & $\beta$-Sitosterol & Twigs of E. robustum & Awouafack et al. (2013a) \\
\hline & & & Roots of $E$. chinense & Thongnest et al. (2013) \\
\hline & 87. & Stigmasterol & Twigs of E. robustum & Awouafack et al. (2013a), \\
\hline & & & Roots of $E$. chinense & Thongnest et al. (201a) \\
\hline & 88. & $\beta$-Sitosterol-3-O- $\beta$-D-glucopyranoside & Whole plant of $E$. glomeratum & Awouafack et al. (2008) \\
\hline & & & Twigs of E. robustum & Awouafack et al. (2013a) \\
\hline \multirow[t]{2}{*}{ Sesquiterpenes } & 89. & Clovanediol & Roots of E. chinense & Thongnest et al. (2013) \\
\hline & 90. & Caryolane-1,9-diol & Roots of $E$. chinense & Thongnest et al. (2013) \\
\hline \multirow[t]{2}{*}{ Monoterpenes } & 91. & Ascaridole & Leaves of $E$. englerianum & Mmbengwa et al. (2009) \\
\hline & 92. & Terpinolene & Leaves of $E$. englerianum & Mmbengwa et al. (2009) \\
\hline
\end{tabular}


TABLE 3 | (Continued) Compounds isolated/identified from Eriosema species (the structure of compounds illustrated in Figure 2).

\begin{tabular}{|c|c|c|c|c|}
\hline Chemical classes & $\mathbf{N}^{\circ}$ & Compounds & $\begin{array}{l}\text { Plant parts } \\
\text { and species }\end{array}$ & References \\
\hline \multicolumn{5}{|l|}{ Other compounds } \\
\hline Toluene derivatives & 93. & O-Cymene & Leaves of $E$. englerianum & Mmbengwa et al. (2009) \\
\hline \multirow{7}{*}{ Benzoic acid Derivatives } & 94. & 4-Hydroxybenzoic acid or p-Hydroxybenzoic acid & Roots of E. tuberosum & Ma et al. (1999) \\
\hline & & & Twigs of E. robustum & Awouafack et al. (2018) \\
\hline & & & Aerial parts of E. laurentii & Ateba et al. (2016a) \\
\hline & 95. & 3,4-Dihydroxybenzoic acid & Aerial parts of $E$. laurentii & Ateba et al. (2016a) \\
\hline & 96. & Vanillic acid & Roots of $E$. tuberosum & Ma et al. (1999) \\
\hline & 97. & 2,6-Dihydroxybenzoic acid & Aerial parts of E. laurentii & Ateba et al. (2016a) \\
\hline & 98. & Eriosematin F or 3,4 dihydro-4-methoxy-naphthalene-2-carboxylic acid & Roots of E. tuberosum & Ma et al. (1999) \\
\hline \multirow[t]{2}{*}{ Fatty acids } & 99. & Eicosanoic acid or arachidic acid & Twigs of E. robustum & Awouafack et al. (2013a) \\
\hline & 100. & Tetratriacontanoic acid & Whole plant of $E$. glomeratum & Awouafack et al. (2008) \\
\hline Glycerol derivative & 101. & 1-O-Heptatriacontanoyl glycerol & Twigs of E. robustum & Awouafack et al. (2013a) \\
\hline Coumarate & 102. & Octaeicosanyl-trans-p-coumarate & Roots of $E$. chinense & Thongnest et al. (2013) \\
\hline Fatty alcohol & 103. & Triacontanol & Whole plant of $E$. glomeratum & Awouafack et al. (2008) \\
\hline \multirow[t]{3}{*}{ Phenols } & 104. & Hydroquinone & Roots of $E$. tuberosum & Ma et al. (1999) \\
\hline & 105. & Arbutin & Roots of $E$. tuberosum & Ma et al. (1999) \\
\hline & 106. & Eriosemaside B or 4-hydroxyphenyl $\beta$-D-apiofuranosyl-(1 $\rightarrow 2)-O-\beta$-D-glucopyranoside & Roots of $E$. tuberosum & Ma et al. (1999) \\
\hline Cerebroside & 107. & Orostachyscerebroside A & Twigs of E. robustum & Awouafack et al. (2013a) \\
\hline
\end{tabular}

*Flavonoids compiled by Awouafack et al. (2015). 


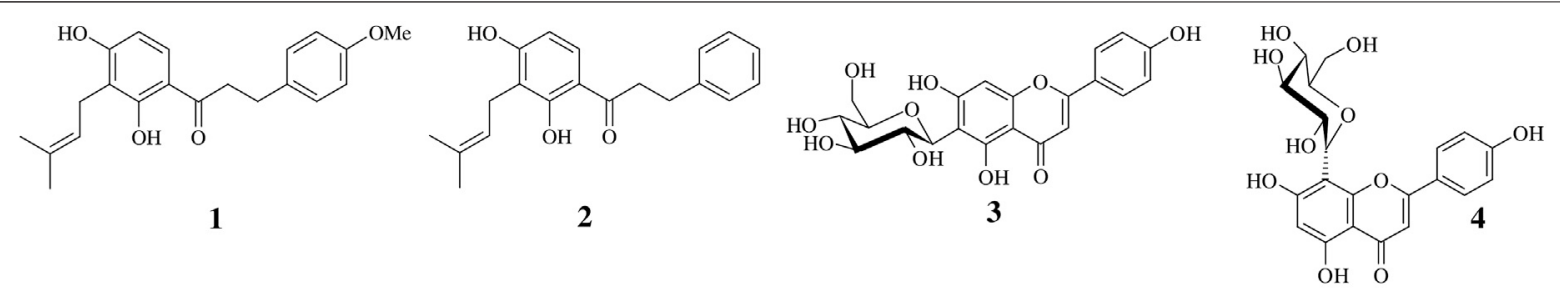

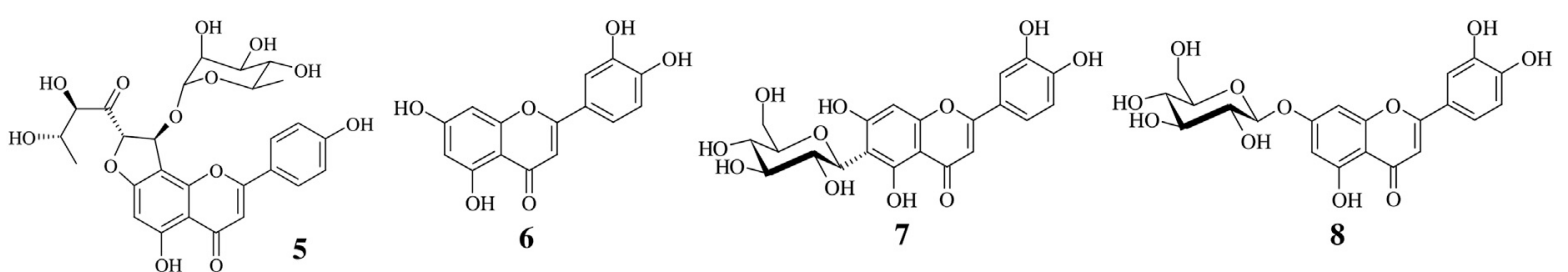

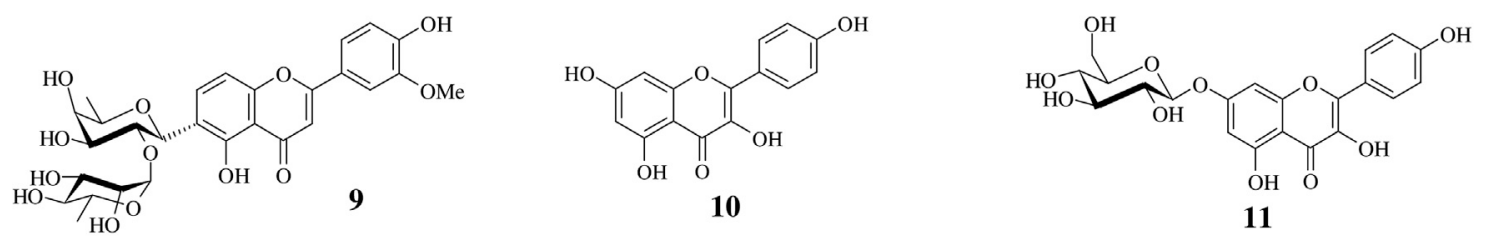

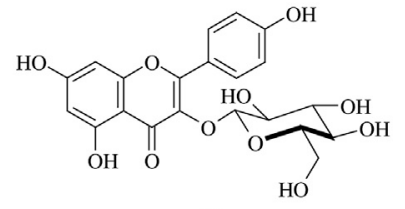

12<smiles>COc1c(O)cc(-c2oc3cc(O)cc(O)c3c(=O)c2O)c(O)c1O</smiles>

16<smiles>CC(C)=CCc1c2c(c(O)c3c(=O)c(O)c(-c4ccc(O)cc4O)oc13)OC(C)(C)C=C2</smiles><smiles>CC(C)=CCc1c2c(c(O)c3c1C(=O)C(O)[C@H](c1ccc(O)cc1)O3)C=CC(C)(C)O2</smiles><smiles>O=c1c(O)c(-c2ccc(O)c(O)c2)oc2cc(O)cc(O)c12</smiles>

13<smiles>COc1ccc(-c2oc3cc(O)cc(O)c3c(=O)c2O)cc1O</smiles>

14<smiles></smiles>

15<smiles>COc1cc(O)c(-c2oc3cc(O)cc(O)c3c(=O)c2O)cc1O</smiles>

17<smiles></smiles>

18<smiles>COc1ccc(-c2oc3c(CC=C(C)C)c4c(c(O)c3c(=O)c2O)C=CC(C)(C)O4)cc1</smiles>

19<smiles>O=C1c2cc(O)c(O)c(O)c2OC(c2ccc(O)cc2)C1O</smiles>

21<smiles>CC(C)=CCc1c2c(c(O)c3c1C(=O)C(O)C(c1ccc(O)cc1O)O3)C=CC(C)(C)O2</smiles><smiles>CC(C)=CCc1c2c(c(O)c3c1O[C@@H](c1ccc(O)cc1)[C@@H](O)C3=O)C=CC(C)(C)O2</smiles>

23

FIGURE 2 | Structures of dihydrochalcones (1-2), flavones (3-9), flavonols (10-20), dihydroflavonols (22-33), flavanones (34-42), isoflavones (43-70),

isoflavanones (71), coumaronochromones (72-74), chromones (75-82), lignans (83-84), terpenoids (85-92) and other compounds (93-107) from Eriosema spp. 
<smiles>COc1ccc([C@H]2Oc3c(CC=C(C)C)c4c(c(O)c3C(=O)[C@@H]2O)C=CC(C)(C)O4)cc1</smiles>

28<smiles>C=C(C)C(O)Cc1c2c(c(O)c3c1C(c1ccc(OC)cc1)C(O)C(O)C3=O)C=CC(C)(C)O2</smiles>

32<smiles>CC(C)=CCc1c2c(c(O)c3c1O[C@H](c1c(O)c(O)cc(-c4ccc(O)cc4O)c1O)CC3=O)C=CC(C)(C)O2</smiles><smiles>CC(C)=CCc1c2c(c(O)c3c1O[C@H](c1ccc(O)cc1O)CC3=O)C=CC(C)(C)O2</smiles>

HO<smiles>Cc1cc(O)c2c(=O)c(-c3ccc(O)cc3O)coc2c1</smiles>

44<smiles>COc1cc(O)cc2occ(-c3ccc(O)cc3)c(=O)c12</smiles>

48<smiles>COc1cc2occ(-c3ccc(O)cc3O)c(=O)c2c(O)c1OC</smiles>

52

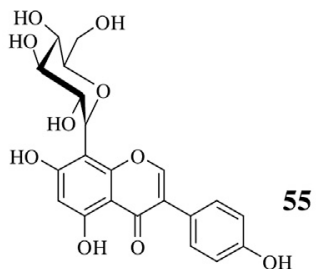<smiles>COc1ccc([C@H]2Oc3c(CC=C(C)C)c4c(c(O)c3C(=O)C2O)C=CC(C)(C)O4)c(O)c1</smiles>

29<smiles>COc1ccc([C@H]2Oc3c(CC4OC4(C)C)c4c(c(O)c3C(=O)[C@@H]2O)C=CC(C)(C)O4)cc1</smiles><smiles>COc1ccc([C@H]2CC(=O)c3c(O)c4c(c(CC=C(C)C)c3O2)OC(C)(C)C=C4)cc1</smiles><smiles>COc1ccc([C@H]2CC(=O)c3c(O)c4c(c(CC=C(C)C)c3O2)OC(C)(C)C=C4)c(O)c1</smiles>

38<smiles>CC(C)=CCc1c(O)cc2c(c1O)C(=O)CC(c1ccccc1)O2</smiles><smiles>COc1cc(O)ccc1-c1coc2cc(O)cc(O)c2c1=O</smiles>

45<smiles>COc1c(O)cc2occ(-c3ccc(O)cc3)c(=O)c2c1O</smiles>

49<smiles>O=c1c(-c2ccc(Cl)cc2)coc2cc(O)cc(O)c12</smiles>

53

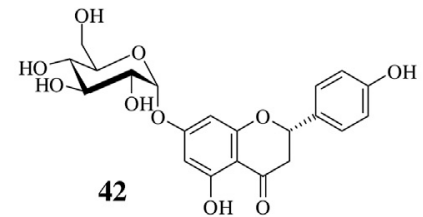<smiles>COc1cc(O)c2c(=O)c(-c3ccc(O)cc3OC)coc2c1</smiles>

46<smiles>COc1cc2occ(-c3ccc(O)cc3)c(=O)c2c(O)c1OC</smiles>

50<smiles>O=c1c(-c2ccc(O)cc2)coc2cc(O)cc(O)c12</smiles>

43<smiles>COc1ccc([C@H]2Oc3c(C[C@H](O)C(C)(C)O)c4c(c(O)c3C(=O)C2O)C=CC(C)(C)O4)cc1</smiles><smiles>CC(C)=CCc1c2c(c(O)c3c1C(=O)C[C@@H](c1cc(O)cc(O)c1)O3)C=CC(C)(C)O2</smiles><smiles>CC(C)=CCc1c2c(c(O)c3c1O[C@H](c1ccc(O)cc1)CC3=O)C=CC(C)(C)O2</smiles>

39

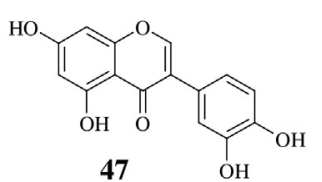<smiles>COc1c(O)cc2occ(-c3ccc(O)cc3O)c(=O)c2c1O</smiles>

51

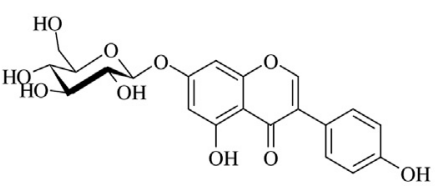

54

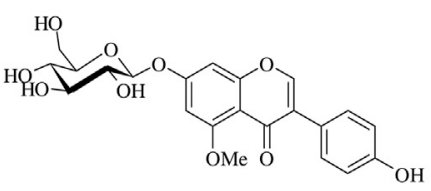

56

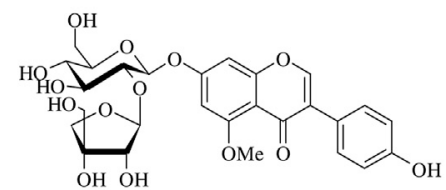

57

Figure 2. | (Continued).

respectively, were also identified for the first time in the genus. Among flavanones, prunin (42) was isolated from the twigs of $E$. robustum (Awouafack et al., 2018).
The isoflavones, genistein (43), 2'-hydroxygenistein (44), genistin (54), genistein-8-C-glucoside (55), $2^{\prime}$-hydroxygenistein7-O-glucoside (61), eriosemaone D (62) and the new compound 


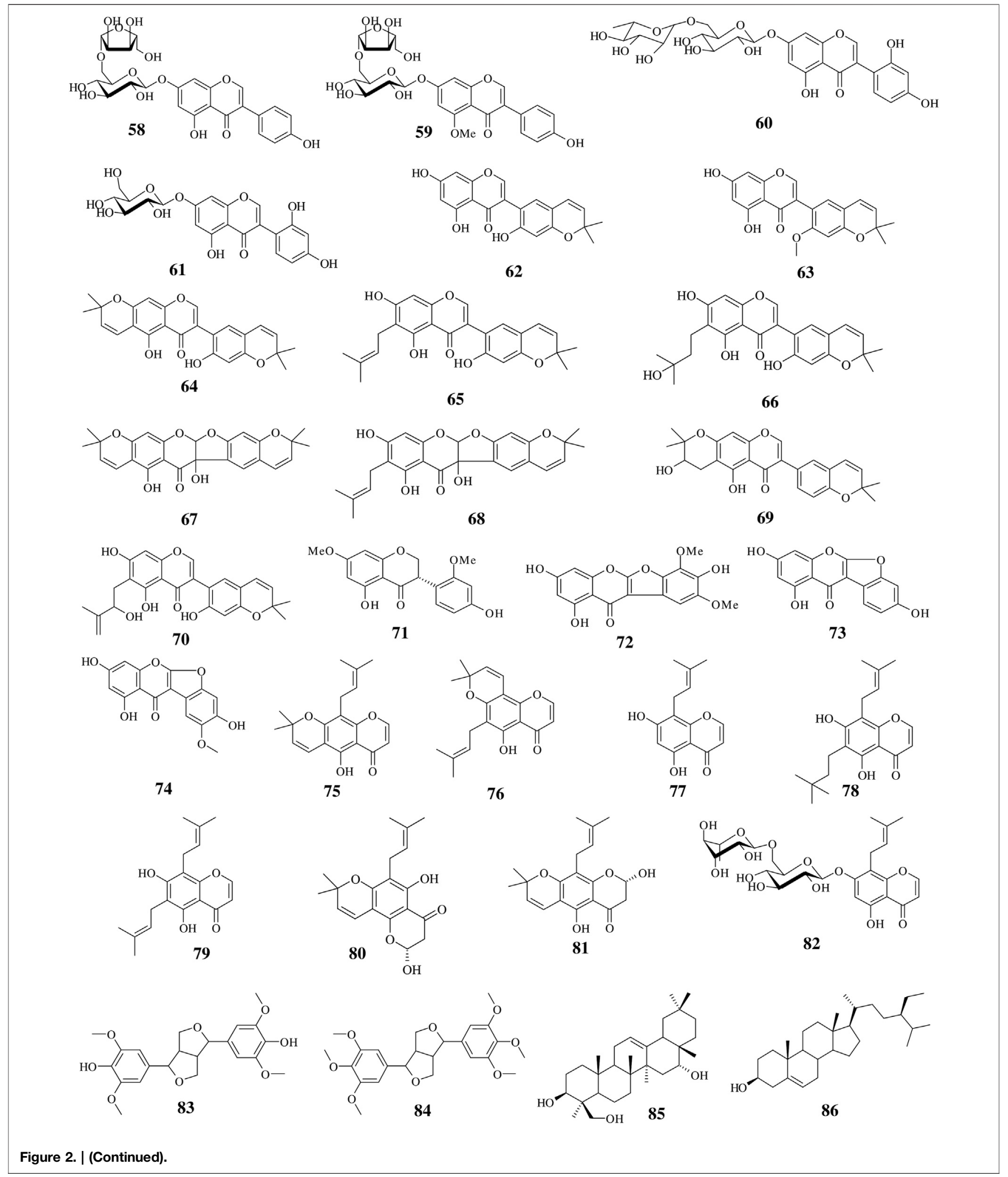

2'-O-methyl-eriosemaone D (63) were identified and/or isolated from E. laurentii (Ateba et al., 2013; 2014b; 2016a; 2016b). Compound 43 was also obtained from the EtOAc-soluble portion of an EtOH extract of the twigs of E. robustum (Awouafack et al., 2018). 5,7,4'-trihdroxy-2'methoxyisoflavone

(45), $4^{\prime}, 5$-dihydroxy-2',7- 
<smiles>CCC(C)C(C)C=CC1CCC2C3CC=C4C[C@H](O)CCC4(C)C3CCC12C</smiles>

87<smiles>CC1=CCC(=C(C)C)CC1</smiles>

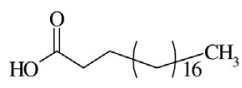

99

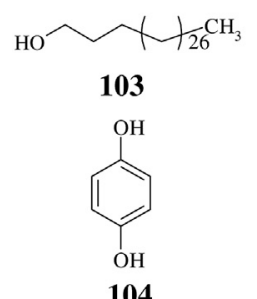

104<smiles>Cc1ccccc1C(C)C</smiles><smiles>CCC(C)C(C)CCC(C)C1CCC2C3CC4=CC(OC5CCC(O)C(CO)CC5O)CCC4(C)C3CCC12C</smiles><smiles>CC1CCC2C(C)CC(O)C2CCC1O</smiles>

89

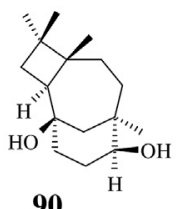

90<smiles>O=C(O)c1ccc(Oc2ccc(O)cc2)c(O)c1</smiles><smiles>COc1ccc(O)c(OC)c1</smiles><smiles>O=C(O)c1c(O)cccc1O</smiles><smiles></smiles>

94<smiles>CCCCCC(=O)OCC(O)CO</smiles>

101

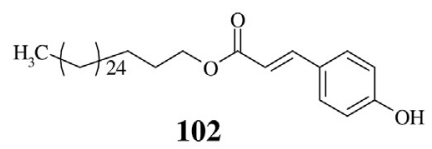

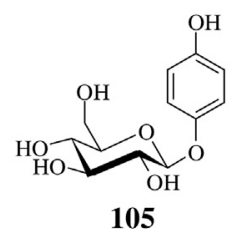

105

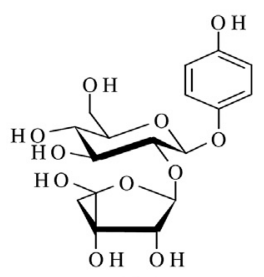

106

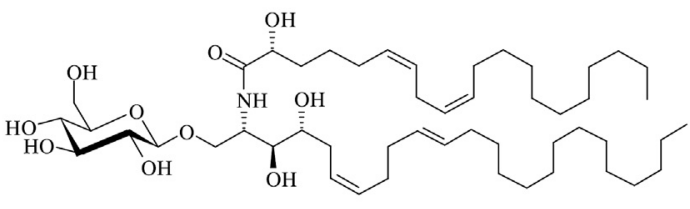

107

Figure 2. | (Continued).

dimethoxyisoflavone (46) and $4^{\prime}, 7^{\prime \prime}$-bisgenistein (53) were obtained from a dichloromethane/methanol $(1: 1 v / v)$ root extract of E. psoraleoides (Wanyama, 2010).

The coumaronochromone named eriocoumaronochromone (72) was isolated for the first time from an $\mathrm{EtOH}$ extract of the twigs of E. robustum, along with desmoxyphyllin A (74) (Awouafack et al., 2018). Lupinalbin A (73) was proven in methanol extracts of the aerial and underground parts of $E$. laurentii (Ateba et al., 2014b; 2016b) and in a dichloromethane/methanol $(1: 1 \quad v / v)$ root extract of $E$. psoraleoides (Wanyama, 2010).

\section{Chromones}

All chromones known in the genus including eriosematin (75), iso-eriosematin (76), eriosematin A-E (77-81) and eriosemaside A (82) were obtained from the roots of $E$. tuberosum (Ma et al., 1995; 1996a; 1996b, 1999). Compound 81 was also identified in an ethanol root extract of E. chinense (Prasad et al., 2017).

\section{Lignans}

Syringaresinol (83) was isolated from the aerial parts of E. laurentii (Ateba et al., 2016a), while yangambin (84) was found in the roots of E. chinense (Sutthivaiyakit et al., 2009).

\section{Terpenoids}

The investigation of a dichloromethane/methanol $(1: 1, \mathrm{v} / \mathrm{v})$ extract of the whole plant of E. glomeratum gave the triterpenoid olean-12-ene-3,16,23-triol (85) and the sterol 3-O- $\beta$-D-sitosterol glucopyranoside (88) (Awouafack et al., 2008). The sterols $\beta$-sitosterol (86) and stigmasterol (87) were reported from an ethanol extract of the twigs of $E$. robustum (Awouafack et al., 2013a) and roots of E. chinense (Thongnest et al., 2013). The sesquiterpenes clovanediol (89) and caryolane-1,9-diol (90) were obtained from roots of $E$. chinense (Thongnest et al., 2013), while the monoterpenes ascaridole (91) and terpinolene (92) have been isolated from the essential oil of leaves of E. englerianum (Mmbengwa et al., 2009).

\section{Other Compounds}

$O$-cymene (93) was obtained as a major phytoconstituent of the essential oil from fresh leaves of $E$. englerianum. 4Hydroxybenzoic acid (94) was isolated from roots of $E$. tuberosum (Ma et al., 1999) and twigs of E. robustum (Awouafack et al., 2018) and from the aerial parts of $E$. laurentii along with 3,4-dihydroxybenzoic acid (95) and 2,6dihydroxybenzoic acid (97) (Ateba et al., 2016a). Vanillic acid (96) and eriosematin F (98) were found in roots of E. tuberosum (Ma et al., 1999). Eicosanoic acid (99) was isolated from twigs of E. robustum (Awouafack et al., 2013a) and tetratriacontanoic acid (100) from a whole plant extract of E. glomeratum (Awouafack et al., 2008). The glycerol derivative 1-O-heptatriacontanoyl glycerol (101) was isolated from twigs of E. robustum (Awouafack et al., 2013a), while octaeicosanyl-transp-coumarate (102) was obtained from the roots of E. chinense 


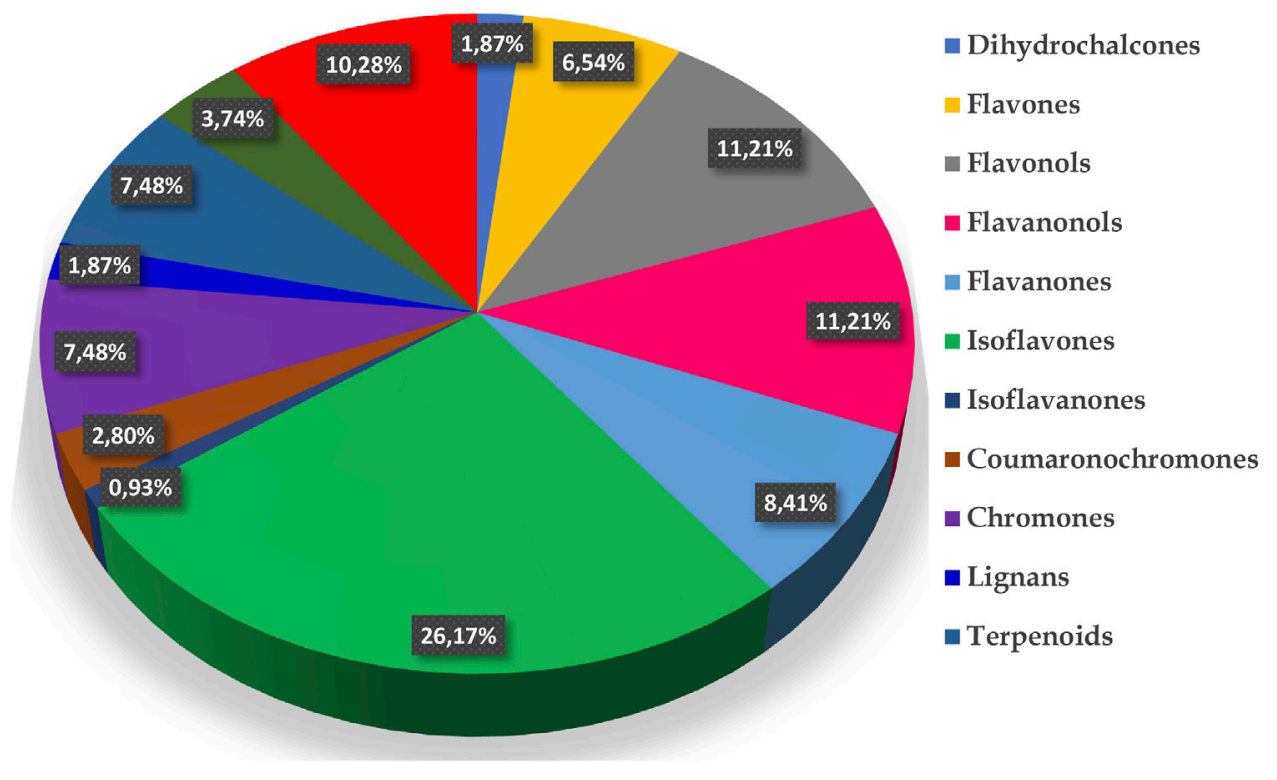

FIGURE 3 | Chemical diversity of Eriosema genus.

(Thongnest et al., 2013). The fatty alcohol triacontanol (103) was isolated from E. glomeratum. Three phenols identified as hydroquinone (104), arbutin (105) and eriosemaside B (106) were isolated from the roots of E. tuberosum (Ma et al., 1999). Twigs of E. robustum contained orostachyscerebroside A (107) (Awouafack et al., 2013a).

Globally the phytochemical investigation of the Eriosema genus led to the isolation and/or identification of 107 compounds from only eight species (5.3\% of the genus) out of ca. 150 (E. chinense, E. englerianum, E. glomeratum, E. kraussianum, E. laurentii, E. robustum, E. tuberosum and E. psoraleoides). Isolated and/or identified constituents are mostly isoflavones (26.2\%), flavonols (11.2\%), flavanonols (11.2\%), flavanones (8.4\%) and chromones (7.5\%) (Figure 3). Awouafack et al. (2015) documented flavonoids from 5 Eriosema species and concluded that the genus represents a rich source of flavonoids, mostly isoflavones which could be considered as markers for the genus and have chemotaxonomic significance. However, given the low number of species investigated until now, the deduction of a chemotaxonomic significance of some of the components seems premature.

\section{PHARMACOLOGY}

Based on their ethnopharmacological use, Eriosema species aroused the interest of the scientific community. In line with this, many studies have been carried out aiming at evaluating or confirming the therapeutic potential of species from this genus. As depicted in Table 4, extracts and constituents from Eriosema species are shown to be responsible for a wide range of pharmacological activities such as aphrodisiac, anti-diarrheal, anti-oxidant, anti-microbial, anti-diabetic/hypoglycemic and estrogenic properties.

\section{Anticancer Activities}

The in vitro antiproliferative or cytotoxic activity of extracts and compounds from E. chinense, E. robustum and E. griseum against various cancer cell lines has been reported suggesting some preliminary potential for cancer treatment. Using the MTT (3(4,5-dimethylthiazolyl-2)-2,5-diphenyltetrazolium bromide) cell viability assay, a hexane root extract of $E$. chinense exhibited a moderate cytotoxic activity against human small-cell lung (NCI$\mathrm{H} 187$ ) $\left(\mathrm{IC}_{50}\right.$ of $\left.12.0 \mu \mathrm{g} / \mathrm{ml}\right)$ and oral epidermal carcinoma (KB) $\left(\mathrm{IC}_{50}\right.$ of $\left.9.9 \mu \mathrm{g} / \mathrm{ml}\right)$ cells. Normal monkey kidney Vero cells were also moderately $\left(\mathrm{IC}_{50}: 5.8 \mu \mathrm{g} / \mathrm{ml}\right.$ ) affected (Sutthivaiyakit et al., 2009). Khonklonginols A (27), F (19) and H (38), lupinifolinol (23), dehydrolupinifolinol (18) and flemichin D (40) from the roots of E. chinense exhibited a strong cytotoxicity $\left(\mathrm{IC}_{50}\right.$ of $2.4-3.9 \mu \mathrm{g} / \mathrm{ml}$ ) toward NCI-H187 cells while khonklonginol B (28), lupinifolin (39) and eriosemaone A (34) with $\mathrm{IC}_{50}$ values of $4.3,6.5$ and $6.0 \mu \mathrm{g} / \mathrm{ml}$, respectively, were moderately cytotoxic (Sutthivaiyakit et al., 2009). Toward KB cells, compounds 23, 27, 28, 39 and 40 displayed a strong cytotoxic effect, while 18, 19, 34 and 38 showed a moderate effect. Compounds 18, 19, 27 and 38 displayed a significant cytotoxicity $\left(\mathrm{IC}_{50}\right.$ of $6.4-11.1 \mu \mathrm{g} / \mathrm{ml}$ ) toward Vero cells.

\section{Antioxidant Activities}

By controlling the formation and scavenging of reactive oxygen and nitrogen species (ROS/RNS) as well as interrupting the related chain reactions and lipid peroxidation, antioxidants constitute the first line of defense against the genesis of various chronic and degenerative diseases. Different in vitro methodologies, including the DPPH (1,1-di-phenyl-2- 
TABLE 4 | Pharmacological properties of Eriosema spp.

\begin{tabular}{|c|c|c|c|c|c|c|}
\hline $\begin{array}{l}\text { Pharmacological } \\
\text { activities }\end{array}$ & Eriosema species & Parts/extracts & Model & $\begin{array}{c}\text { Dosage/concentration } \\
\text { and } \\
\text { route of } \\
\text { administration in } \\
\text { the animal } \\
\text { studies }\end{array}$ & Results & References \\
\hline Anticancer & E. chinense & Roots/hexane extract & $\begin{array}{l}\text { In vitro: MTT assay } \\
\text { Small-cell lung (NCI-H187), oral epidermal } \\
\text { carcinoma (KB) human cancer cells }\end{array}$ & & $\begin{array}{l}\text { Moderate activity }\left(\mathrm{IC}_{50}: 12.0 \mu \mathrm{g} / \mathrm{ml}\right) \\
\text { against small-cell lung and oral } \\
\text { epidermal carcinoma }\left(\mathrm{IC}_{50}: 9.9 \mu \mathrm{g} / \mathrm{ml}\right) \\
\text { human cells }\end{array}$ & $\begin{array}{l}\text { Sutthivaiyakit et al. } \\
\text { (2009) }\end{array}$ \\
\hline \multirow[t]{3}{*}{ Anti-oxidant } & E.englerianum & Leaves/essential oil & In vitro: $\beta$-carotene bleaching assay & & Color retention zone of $14.4 \mathrm{~mm}$ & $\begin{array}{l}\text { Mmbengwa et al. } \\
(2009)\end{array}$ \\
\hline & E. chinense & Roots/EtOH extract & $\begin{array}{l}\text { In vitro: PFRAP assay } \\
\text { In vitro: } \mathrm{DPPH}, \mathrm{H}_{2} \mathrm{O}_{2} \text { and nitric oxide radical } \\
\text { scavenging assays }\end{array}$ & & $\begin{array}{l}\text { Reducing power of } 0.263 \mu \mathrm{g} / \mathrm{ml} \\
\text { Scavenging activities of differing degree } \\
\text { with } \mathrm{IC}_{50} \text { values of } 146,221,233 \text { and } \\
170 \mu \mathrm{g} / \mathrm{ml} \text {, respectively }\end{array}$ & Prasad et al. (2013b) \\
\hline & E. robustum & Twigs/EtOH extract & DPPH assay & & $\begin{array}{l}\text { Scavenging activity with } \mathrm{IC}_{50} \geq \\
1.13 \mathrm{mg} / \mathrm{ml}\end{array}$ & $\begin{array}{l}\text { Awouafack et al. } \\
\text { (2013b) }\end{array}$ \\
\hline \multirow[t]{3}{*}{ Estrogenic } & E. laurentii & Aerial parts $/ \mathrm{MeOH}$ extract & In vitro: ERa yeast two-hybrid assay & $1-83 \mu \mathrm{g} / \mathrm{ml}$ & $\begin{array}{l}\text { Concentration-dependent } \\
\beta \text {-galactosidase activity }\end{array}$ & Ateba et al. (2013) \\
\hline & & & In vivo: Ovariectomized Wistar rats & $\begin{array}{l}50,100 \text { and } 200 \mathrm{mg} / \mathrm{kg} \\
\text { daily for } 3 \text { days and } \\
9 \text { weeks p.o }\end{array}$ & $\begin{array}{l}\text { Vaginal stratification and cornification, no } \\
\text { effect on uterus }\end{array}$ & Ateba et al. (2013) \\
\hline & & $\begin{array}{l}\text { Underground parts/ } \\
\mathrm{MeOH} \text { extract }\end{array}$ & In vivo: Ovariectomized Wistar rats & $\begin{array}{l}50,100 \text { and } 200 \mathrm{mg} / \mathrm{kg} \\
\text { daily for } 3 \text { days p.o }\end{array}$ & No effect on uterus and vagina & Ateba (2014c) \\
\hline $\begin{array}{l}\text { Aryl hydrocarbon } \\
\text { agonistic }\end{array}$ & E. laurentii & $\begin{array}{l}\text { Aerial parts/MeOH extract } \\
\text { Underground parts/ } \\
\mathrm{MeOH} \text { extract }\end{array}$ & In vitro: AhR yeast $\beta$-galactosidase assay & $\begin{array}{l}1-83 \mu \mathrm{g} / \mathrm{ml} \\
1-125 \mu \mathrm{g} / \mathrm{ml}\end{array}$ & $\begin{array}{l}\text { Concentration-dependent AhR } \\
\beta \text {-galactosidase activity }\end{array}$ & $\begin{array}{l}\text { Ateba et al. (2013) } \\
\text { Ateba et al. (2016b) }\end{array}$ \\
\hline Anti-osteoporosis & E. laurentii & Aerial parts $/ \mathrm{MeOH}$ extract & In vivo: Ovariectomized Wistar rats & $\begin{array}{l}\text { 50, } 100 \text { and } 200 \mathrm{mg} / \mathrm{kg} \\
\text { daily for } 9 \text { weeks p.o }\end{array}$ & $\begin{array}{l}\uparrow \text { dry femur weight, } \uparrow \text { serum and femur } \\
\text { calcium levels } \\
\downarrow \text { serum inorganic phosphorus levels, } \uparrow \\
\text { femur inorganic phosphorus levels }\end{array}$ & Ateba et al. (2013) \\
\hline \multirow[t]{3}{*}{$\begin{array}{l}\text { Hypoglycemic and anti- } \\
\text { diabetic }\end{array}$} & E. kraussianum & $\begin{array}{l}\text { Rootstock/hydro- } \\
\text { alcoholic extract }\end{array}$ & $\begin{array}{l}\text { In vivo: normal Wistar and streptozotocin- } \\
\text { induced diabetic Wistar rats }\end{array}$ & $\begin{array}{l}40,80,160 \text { and } \\
320 \mathrm{mg} / \mathrm{kg} \text { p.o. (single } \\
\text { dose) }\end{array}$ & $\begin{array}{l}\downarrow \text { Fasting blood glucose levels in dose- } \\
\text { dependent manner from } 2 \text { to } 8 \mathrm{~h} \text { after } \\
\text { treatment with peak effects } 4 \mathrm{~h} \text { after }\end{array}$ & Ojewole et al. (2007) \\
\hline & E. psoraleoides & $\begin{array}{l}\text { Leaves } / \mathrm{H}_{2} \mathrm{O} \text { and } \mathrm{EtOH} \\
\text { extracts }\end{array}$ & In vivo: alloxan-induced diabetic Wistar rats & $\begin{array}{l}200 \text { and } 400 \mathrm{mg} / \mathrm{kg} \text { daily } \\
\text { for } 7 \text { days p.o }\end{array}$ & $\downarrow$ Fasting blood glucose levels from day 6 & $\begin{array}{l}\text { Nduka et al. (2018), } \\
\text { Nduka et al. (2019) }\end{array}$ \\
\hline & & $\begin{array}{l}\text { Leaves } / n \text {-hexane extract, } \\
n \text {-hexane-DCM and DCM } \\
\text { fractions }\end{array}$ & In vivo: alloxan-induced diabetic Wistar rats & $\begin{array}{l}200 \mathrm{mg} / \mathrm{kg} \text { daily for } \\
7 \text { days p.o }\end{array}$ & $\begin{array}{l}\downarrow \text { Fasting blood glucose levels from } \\
\text { day 2-7 }\end{array}$ & Elechi et al. (2019) \\
\hline $\begin{array}{l}\text { Cardiovascular } \\
\text { protection }\end{array}$ & E. laurentii & Aerial parts $/ \mathrm{MeOH}$ extract & $\begin{array}{l}\text { In vivo: postmenopause-like model } \\
\text { (ovariectomized Wistar rats) }\end{array}$ & $\begin{array}{l}\text { 50, } 100 \text { and } 200 \mathrm{mg} / \mathrm{kg} \\
\text { daily for } 9 \text { weeks p.o }\end{array}$ & $\begin{array}{l}\downarrow \text { Total cholesterol (TC) and LDL levels } \\
\uparrow \mathrm{HDL} \\
\downarrow \mathrm{TC} / \mathrm{HDL} \text { and LDL/HDL ratios, } \\
\text { atherogenic plasma index }\end{array}$ & Ateba et al. (2013) \\
\hline Acetylcholinesterase & E. cordatum & Roots $/ \mathrm{H}_{2} \mathrm{O}$ extract & In vitro: Acetylcholinesterase inhibition & & Low activity ( $\left(\mathrm{I}_{50}\right.$ of $\left.757 \mu \mathrm{g} / \mathrm{ml}\right)$ & Ndhlala et al. (2011) \\
\hline
\end{tabular}


TABLE 4 | (Continued) Pharmacological properties of Eriosema spp.

Pharmacological Eriosema species Parts/extracts

activities

Model

Dosage/concentration

and

Results

administration in

the animal

studies

Anti-microbia

Eriosema campestre Leaves $/ \mathrm{MeOH}$ extract
var. campestre

In vitro

Trichophyton rubrum

Trichophyton mentagrophytes

Microsporum gypseum

Epidermophyton floccosum

Candida albicans ATCC 90028

Candida krusei ATCC 6258

Candida glabrata HCCGL01

Candida tropicalis ATCC 750

Eriosema campestre Leaf/MeOH extract

Candida parapsilosis ATCC22019

var. macrophyllum

In vitro

Trichophyton rubrum

Trichophyton mentagrophytes

Microsporum gypseum

Epidermophyton floccosum

Candida albicans ATCC 90028

Candida krusei ATCC 6258

Candida glabrata HCCGLO1

Candida tropicalis ATCC 750

E. cordatum

Roots/petroleum ether, DCM, EtOH and $\mathrm{H}_{2} \mathrm{O}$

Candida parapsilosis ATCC22019

In vitro

extracts

Staphylococcus aureus

Bacillus subtilis

Escherichia coli

Klebsiella pneumonia

E. chinense

Roots/EtOH extract

In vitro

Escherichia coli ATCC25922,

Shigella flexneri ATCC12022

Pseudomonas aeruginosa ATCC27893

Staphylococcus aureus ATCC25323

Salmonella typhi

Shigella dysenteriae

Proteus vulgaris

Klebsiella pneumoniae

Shigella boydii

Bacillus cereus

Enterococcus faecalis tested Candida strains
$500 \mu \mathrm{g} / \mathrm{ml}$

Fungistatic toward T. rubrum, $T$.

mentagrophytes and $E$. floccosum.

Inactive against $M$. gypseum and all

$500 \mu \mathrm{g} / \mathrm{ml}$

Fungistatic toward T. mentagrophytes and $E$. floccosum. Inactive against $T$.

rubrum, M. gypseum and all tested

Candida strains

MIC of $390 \mu \mathrm{g} / \mathrm{ml}$ with ethanolic extract Ndhlala et al. (2011) MIC of $195 \mu \mathrm{g} / \mathrm{ml}$ against S. aureus and

B. subtilis, and of $390 \mu \mathrm{g} / \mathrm{ml}$ against

E. coli and K. pneumoniae with

dichloromethane extract

Petroleum ether and $\mathrm{H}_{2} \mathrm{O}$ extracts not

active

MICs $>195 \mu \mathrm{g} / \mathrm{m}$

Prasad et al. (2013)

(Continued on following page) 
TABLE 4 | (Continued) Pharmacological properties of Eriosema spp.

Pharmacological Eriosema species Parts/extracts

Dosage/concentration

and

route of

administration in

the animal

studies

\begin{tabular}{|c|c|c|c|c|c|}
\hline E. englerianum & Leaves/essential oil & $\begin{array}{l}\text { In vitro } \\
\text { Staphylococcus aureus } \\
\text { Escherichia coli } \\
\text { Pseudomonas aeruginosa } \\
\text { Bacillus subtilis } \\
\text { Klebsiella pneumoniae } \\
\text { Proteus vulgaris } \\
\text { Clostridium sporogenes } \\
\text { Acinetobacter calcoaceticus } \\
\text { Candida albicans } \\
\text { Aspergillus niger } \\
\text { Aspergillus flavus }\end{array}$ & $1,2,5$ and $10 \mu \mathrm{L} / \mathrm{ml}$ & $\begin{array}{l}\mathrm{IZ} \geq 7 \mathrm{~mm} \text { at } 5 \mu \mathrm{L} / \mathrm{ml} \text { (A. calcoaceticus) } \\
\text { and } 10 \mu \mathrm{L} / \mathrm{ml}(\mathrm{C} \text {. sporogenes and } A \text {. } \\
\text { calcoaceticus) } \\
\downarrow \text { growth of C. albicans (24.2-42.6\%), } \\
\text { Aspergillus niger (34.7-49.7\%) and } \\
\text { Aspergillus flavus (27.5-39.3\%) }\end{array}$ & $\begin{array}{l}\text { Mmbengwa et al. } \\
\text { (2009) }\end{array}$ \\
\hline Eriosema glabrum & Leaves/MeOH extract & $\begin{array}{l}\text { In vitro } \\
\text { Trichophyton rubrum } \\
\text { Trichophyton mentagrophytes } \\
\text { Microsporum gypseum } \\
\text { Epidermophyton floccosum } \\
\text { Candida albicans ATCC } 90028 \\
\text { Candida krusei ATCC } 6258 \\
\text { Candida glabrata HCCGL01 } \\
\text { Candida tropicalis ATCC } 750 \\
\text { Candida parapsilosis ATCC22019 }\end{array}$ & $500 \mu \mathrm{g} / \mathrm{ml}$ & $\begin{array}{l}\text { Fungicidal toward } T \text {. mentagrophytes } \\
\text { and } E \text {. floccosum. Inactive against } T \text {. } \\
\text { rubrum, M. gypseum and all tested } \\
\text { Candida strains }\end{array}$ & de Morais et al. (2017) \\
\hline E. glomeratum & $\begin{array}{l}\text { Stem and leaves } / \mathrm{MeOH}- \\
\text { DCM extract }\end{array}$ & $\begin{array}{l}\text { In vitro } \\
\text { Staphylococcus aureus ATCC } 25923 \\
\text { Klebsiella pneumonia ATCC } 13883 \\
\text { Morexella catarrhalis ATCC } 14468 \\
\text { Mycobacterium smegmatis ATCC23246 } \\
\text { Mycobacterium aurum NCTC10437 }\end{array}$ & & $\begin{array}{l}\text { Stem extract: MICs of } 500,1000,1000 \text {, } \\
65 \text {, and } 50 \mu \mathrm{g} / \mathrm{ml} \text {, respectively } \\
\text { Leaf extract: MICs of } 1,000,500 \text {, } \\
1,000,130 \text { and } 1,000 \mu \mathrm{g} / \mathrm{ml} \text {, respectively }\end{array}$ & $\begin{array}{l}\text { Fomogne-Fodjo et al. } \\
\text { (2014) }\end{array}$ \\
\hline $\begin{array}{l}\text { Eriosema } \\
\text { heterophyllum }\end{array}$ & Leaves/MeOH extract & $\begin{array}{l}\text { In vitro } \\
\text { Trichophyton rubrum } \\
\text { Trichophyton mentagrophytes } \\
\text { Microsporum gypseum } \\
\text { Epidermophyton floccosum } \\
\text { Candida albicans ATCC } 90028 \\
\text { Candida krusei ATCC } 6258 \\
\text { Candida glabrata HCCGL01 } \\
\text { Candida tropicalis ATCC } 750 \\
\text { Candida parapsilosis ATCC22019 }\end{array}$ & $500 \mu \mathrm{g} / \mathrm{ml}$ & $\begin{array}{l}\text { Fungistatic toward } T \text {. rubrum, } T \text {. } \\
\text { mentagrophytes and } M \text {. gypseum. } \\
\text { Fungicidal against } E \text {. floccosum. Inactive } \\
\text { against, and all tested Candida strains }\end{array}$ & de Morais et al. (2017) \\
\hline
\end{tabular}


TABLE 4 | (Continued) Pharmacological properties of Eriosema spp.

Pharmacological Eriosema species Parts/extracts

activities

Eriosema species Marts/extracts Model

Model

Dosage/concentration

and

Results

References

administration in

the animal

studies

In vitro

$500 \mu \mathrm{g} / \mathrm{ml}$

Inactive

de Morais et al. (2017)

Trichophyton rubrum

Trichophyton mentagrophytes

Microsporum gypseum

Epidermophyton floccosum

Candida albicans ATCC 90028

Candida krusei ATCC 6258

Candida glabrata HCCGL01

Candida tropicalis ATCC 750

Candida parapsilosis ATCC22019

E. montanum

Leaves/polar fraction of

an $\mathrm{EtOH}$ extract

In vitro

Bacillus cereus ATCC 14579,

Enterobacter cloacae ATCC 13047

Escherichia coli ATCC 8739

Klebsiella pneumoniae ATCC 13883

Mycobacterium fortuitum ATCC 6841

Proteus vulgaris ATCC 13315

Pseudomonas aeruginosa ATCC 15442

Salmonella typhimurium ATCC 13311

Staphylococcus aureus ATCC 6538

Streptococcus pyogenes ATCC 12344

Candida albicans ATCC 10231

Trichophyton rubrum RV 58125

Epidermophyton floccosum RV 71625

Microsporum canis RV 66973

E. psoraleoides

Bark and wood of twigs/ In vitro

$\mathrm{MeOH}$ extract Streptococcus mutans HG982

Actinomyces viscosus HG485

Candida albicans HG392

Stem bark $/ \mathrm{MeOH}$ extract In vitro

Candida albicans

In vitro

Leaves $/ \mathrm{MeOH}$ extract

and its n-hexane and

Escherichia coli ATCC35219,

ethyl acetate fractions

Staphylococcus aureus ATCC25923

Clinical isolates of Staphylococcus aureus

Proteus vulgaris

Klebsiella erogenes

Pseudomonas aeruginosa

Escherichia coll
Inactive

Runyoro et al. (2006)

Inactive ( $\mathrm{MIC}>1.26 \mathrm{mg} / \mathrm{ml}$, except n-hexane fraction (MIC of $150 \mu \mathrm{g} / \mathrm{ml}$ ) Elechi and lgboh toward S. aureus ATCC
Cos et al. (2002b)

Khan et al. (2000)

ued on following page) 
TABLE 4 | (Continued) Pharmacological properties of Eriosema spp.

Pharmacological Eriosema species Parts/extracts

activities

Eriosema species

arts/extracts

Model

Dosage/concentration

Results

References

and

route of

administration in

the animal

studies

E. robustum

Twigs/EtOH extract

In vitro

Staphylococcus aureus ATCC29213

Enterococcus faecalis ATCC29212

Pseudomonas aeruginosa

ATCC27853

Escherichia coli ATCC25922

Candida albicans ATCC10231

Candida albicans

Cryptococcus neoformans

Eriosema

Leaves $/ \mathrm{MeOH}$ extract

Aspergillus fumigatus

tacuaremboense

In vitro

Trichophyton rubrum

Trichophyton mentagrophytes

Microsporum gypseum

Epidermophyton floccosum

Candida albicans ATCC 90028

Candida krusei ATCC 6258

Candida glabrata HCCGLO1

Candida tropicalis ATCC 750

Leaves/polar fraction of

an $\mathrm{EtOH}$ extract

Candida parapsilosis ATCC22019

In vitro

Human immunodeficiency virus type-1

infected MT-4 cells

In vitro

Herpes simplex virus type 1 and semliki

forest virus A7

Poliomyelitis virus type 1 strain 1 A/S3

Coxsackie B2 virus

Vesicular stomatitis virus T2

Measles Edmonston A

Anti-diarrheal

E. chinense

Roots/EtOH extract and

its chloroform fraction
Measles Edmonston

In vivo: castor oil-induced diarrhea rats
MIC of $310 \mu \mathrm{g} / \mathrm{ml}$ toward $P$. aeruginosa

$500 \mu \mathrm{g} / \mathrm{m}$

$375 \mu \mathrm{g} / \mathrm{ml}$

$375 \mu \mathrm{g} / \mathrm{ml}$

$187.5 \mu \mathrm{g} / \mathrm{ml}$

$187.5 \mu \mathrm{g} / \mathrm{ml}$

$750 \mu \mathrm{g} / \mathrm{ml}$

Extract: $400 \mathrm{mg} / \mathrm{kg} \mathrm{p.o}$

Fraction: $100 \mathrm{mg} / \mathrm{kg} \mathrm{p.O}$

In vivo: enteropathogenic Escherichia coliinduced diarrhea
E. faecalis and $E$. coli

MIC of $160 \mu \mathrm{g} / \mathrm{ml}$ toward $A$. fumigatus

$\mathrm{MIC}$ of $630 \mu \mathrm{g} / \mathrm{ml}$ against $C$. neoforman

MIC of $1250 \mu \mathrm{g} / \mathrm{ml}$ ) toward C. albicans

and C. albicans ATCC

Fungistatic toward E. floccosum

Inactive against $T$. rubrum, $T$.

mentagrophytes, M. gypseum and all

tested Candida strains

Inactive $\left(E_{50}>166 \mu \mathrm{g} / \mathrm{ml}\right)$

Cos et al. (2002a)

Awouafack et al.

(2013a)

de Morais et al. (2017)

Cos et al. (2002b)

RF of $10^{4}$

$\mathrm{RF}$ of $10^{3}$

$\mathrm{RF}$ of $10^{3}$

RF of $10^{2}$

RF of $10^{2}$

$\downarrow$ normal fecal excretion rate and water Prasad et al. (2013c) content

Delayed onset of diarrhea

$\downarrow$ mean defecation, peristaltic index intestinal fluid volume, prostaglandine

E2-induced enteropooling

Significant recovery of $\mathrm{Na}^{+}$and $\mathrm{K}^{+}$levels Prevention of alterations of nitric oxide,

TBARS, SOD and catalase activities

$\downarrow$ total number of stools, total number of Parmar et al. (2019a) diarrheal stools, weight of stools, mean

defecation rate and water content

$\uparrow \mathrm{Na}^{+} / \mathrm{K}^{+}$-atpase activity, \intestinal

secretion
Extract: 100 and

$200 \mathrm{mg} / \mathrm{kg} \mathrm{p.o}$

Fraction: 50 and

$100 \mathrm{mg} / \mathrm{kg}$ p.o 
TABLE 4 | (Continued) Pharmacological properties of Eriosema spp.

\begin{tabular}{|c|c|c|c|c|c|c|}
\hline $\begin{array}{l}\text { Pharmacological } \\
\text { activities }\end{array}$ & Eriosema species & Parts/extracts & Model & $\begin{array}{c}\text { Dosage/concentration } \\
\text { and } \\
\text { route of } \\
\text { administration in } \\
\text { the animal } \\
\text { studies }\end{array}$ & Results & References \\
\hline \multirow[t]{2}{*}{ Anti-inflammatory } & E. campestre & Roots/DCM-EtOH extract & In vitro: Peripheral blood mononuclear cells & $6.25,12.5$ and $25 \mu \mathrm{g} / \mathrm{ml}$ & $\begin{array}{l}\downarrow \mathrm{T} \text { lymphocytes, including } \mathrm{CD} 4^{+} \text {and } \\
\mathrm{CD} 8^{+} \text {cells } \\
\downarrow \text { IL-2 levels in the supernatant of the cell } \\
\text { cultures }\end{array}$ & Santos et al. (2016) \\
\hline & E. cordatum & $\begin{array}{l}\text { Roots/petroleum ether, } \\
\text { DCM, EtOH and } \mathrm{H}_{2} \mathrm{O} \\
\text { extracts }\end{array}$ & In vitro: Cyclooxygenase inhibition & $\begin{array}{l}250 \mu \mathrm{g} / \mathrm{ml} \text { for organic } \\
\text { extracts } \\
2 \mathrm{mg} / \mathrm{ml} \text { for the aqueous } \\
\text { extract }\end{array}$ & $\begin{array}{l}\text { Inhibition of COX-1 activity by } 13-34 \% \\
\text { Inhibition of COX-2 activity by } 6-35 \%\end{array}$ & Ndhlala et al. (2011) \\
\hline \multirow[t]{2}{*}{ Anthelmintic } & E. griseum & Leaves/EtOH extract & $\begin{array}{l}\text { In vitro: Echinostoma caproni, Schistosoma } \\
\text { mansoni, Ancylostom aceylanicum, } \\
\text { Heligmosomoides bakeri and Trichuris } \\
\text { muris }\end{array}$ & & $\begin{array}{l}\text { MLC of } 20 \mu \mathrm{g} / \mathrm{ml} \text { against third-stage } \\
\text { larvae of } H . \text { bakeri } \\
\text { MLC of } 20 \mu \mathrm{g} / \mathrm{ml} \text { toward newly } \\
\text { transformed S. mansoni schistosomula } \\
\text { MLC of } 40 \mu \mathrm{g} / \mathrm{ml} \text { against adult } \\
\text { S. mansoni } \\
\text { Moderate activity against third-stage } \\
\text { larvae of } A \text {. ceylanicum } \\
\text { no activity on adult } E \text {. caproni and } \\
\text { T. muris }\end{array}$ & Koné et al. (2012) \\
\hline & & & $\begin{array}{l}\text { In vivo: mice harboring adult S. mansoni, } \\
\text { E. caproni and T. muris }\end{array}$ & $\begin{array}{l}\text { Single oral dose of } 400 \text { or } \\
800 \mathrm{mg} / \mathrm{kg} 49 \text { days post- } \\
\text { infection }\end{array}$ & $\begin{array}{l}49.5 \% \text { reduction of worm burden } \\
\text { against adult } S \text {. mansoni at } 400 \mathrm{mg} / \mathrm{kg} \\
\text { No activity against } E \text {. caproni and } \\
T \text {. muris at } 800 \text { and } 400 \mathrm{mg} / \mathrm{kg} \text {, } \\
\text { respectively }\end{array}$ & \\
\hline
\end{tabular}

${ }^{a}$ !: decrease or inhibition; $\uparrow:$ increase; DPPH (1,1-di-phenyl-2-picrylhydrazy), DCM: dichloromethane; PFRAP: potassium ferricyanide antioxidant power, IC 50 : concentration of a substance required for $50 \%$ of its maximal inhibitory effect, IZ: diameter of inhibition zone, MIC: minimum inhibitory concentration, MLC: minimum lethal concentration, p. o.: per os, RF: reduction factor of the viral titer, SOD: superoxide dismutase, TBARS: thiobarbituric acid reactive substances. 
picrylhydrazyl), nitric oxide radical and $\mathrm{H}_{2} \mathrm{O}_{2}$ scavenging assays, potassium ferricyanide antioxidant power (PFRAP), hydroxyl radical antioxidant capacity (HORAC), thiobarbituric acid reactive substances (TBARS) and the $\beta$-carotene bleaching test were used to evaluate the radical scavenging and antioxidant potential of members of the Eriosema genus.

Using a $\beta$-carotene bleaching assay, essential oil from fresh leaves of E. englerianum containing 3.65\% ascaridole (91), 7.86\% terpinolene (92) and $88.50 \% \mathrm{O}$-cymene (93) showed anti-oxidant activity with a color retention zone of $14.4 \mathrm{~mm}$ (Mmbengwa et al., 2009).

In the study by Prasad et al. (2013b), an ethanolic root extract of E. chinense (EEC) displayed a total antioxidant capacity of $90.2 \mu \mathrm{g} / \mathrm{ml}$ ascorbic acid equivalents. The PFRAP of the extract and the standard ascorbic acid was 0.26 and $0.42 \mu \mathrm{g} / \mathrm{ml}$, respectively. The $\mathrm{IC}_{50}$ values of the extract for $\mathrm{DPPH}$, hydrogen peroxide, nitric oxide and hydroxyl radical were 146.3, 221.0, 232.9 and $170.2 \mu \mathrm{g} / \mathrm{ml}$, respectively, whereas those of the standards ascorbic acid (DPPH assay), rutin $\left(\mathrm{H}_{2} \mathrm{O}_{2}\right.$ and nitric oxide) and butylated hydroxyanisole (hydroxyl) were 79.1, 82.9, 76.4 and $71.9 \mu \mathrm{g} / \mathrm{ml}$, respectively. The antioxidant capacity of fractions (hexane, chloroform, ethyl acetate and aqueous) of EEC was also evaluated. A total antioxidant capacity of $76.0,97.4,89.5$ and $44.6 \mu \mathrm{g} / \mathrm{ml}$ ascorbic acid equivalents, respectively, was determined. In contrast, very low DPPH ( $\mathrm{IC}_{50} 163.1-312.1 \mu \mathrm{g} / \mathrm{ml}$ ), nitric oxide $\left(\mathrm{IC}_{50} 201.8-372.2 \mu \mathrm{g} / \mathrm{ml}\right)$ and $\mathrm{H}_{2} \mathrm{O}_{2}\left(\mathrm{IC}_{50} \geq 187.7 \mu \mathrm{g} / \mathrm{ml}\right)$ radical scavenging activities as well as a low HORAC $\left(\mathrm{IC}_{50} \geq\right.$ $154.4 \mu \mathrm{g} / \mathrm{ml}$ ) were observed for these fractions (Prasad et al., 2013a). Thongnest et al. (2013) evaluated the antioxidant activity of 11 flavonoids from the roots of E. chinense using a DPPH method. The most pronounced effects, close to the standard butylated hydroxytoluene $\left(\mathrm{IC}_{50}\right.$ of $\left.39 \mu \mathrm{M}\right)$, were observed with compounds $\mathbf{1 0}\left(\mathrm{IC}_{50}\right.$ of $\left.28 \mu \mathrm{M}\right)$ and $20\left(\mathrm{IC}_{50}\right.$ of $35 \mu \mathrm{M}$ ), while $11,12,23-25,27,33,40$ and 49 with $\mathrm{IC}_{50} \geq$ $252 \mu \mathrm{M}$, were inactive. In rats, a single oral pretreatment (30 min before castor oil challenge) with EEC (at $400 \mathrm{mg} / \mathrm{kg}$ ), its chloroform fraction (at $100 \mathrm{mg} / \mathrm{kg}$ ), and compounds 39 $(10 \mathrm{mg} / \mathrm{kg})$ and $81(10 \mathrm{mg} / \mathrm{kg})$ prevented the alterations of nitric oxide, TBARS (lipid peroxidation), superoxide dismutase and catalase activities in the castor oil-mediated diarrhea model (Prasad et al., 2013c; Prasad et al., 2017). Compound 81 ( 5 and $10 \mathrm{mg} / \mathrm{kg}$ ) restored the oxidative status (nitric oxide level, SOD and CAT activities) in the enteropathogenic Escherichia coli-induced diarrhea rat model.

The anti-oxidant activity of an ethanol extract of twigs of $E$. robustum, its fractions and the isolated compounds 16 and 17, 41 and 101 resulted in unphysiological $\mathrm{IC}_{50}$ values $(\geq 1.13 \mathrm{mg} / \mathrm{ml})$ using a DPPH method (Awouafack et al., 2013b). Using the same assay, compounds 5 and 72 displayed $\mathrm{IC}_{50}$ values of 340.3 and $524.6 \mu \mathrm{M}$, respectively, while that of ascorbic acid was $35.9 \mu \mathrm{M}$ (Awouafack et al., 2018).

The antioxidant capacity of Eriosema species was evaluated in vitro by different methods addressing different mechanisms of action. Each of them has its strengths and weaknesses (Bibi Sadeer et al., 2020). The plant extracts and products are mixtures of compounds that can display their antioxidant activity through different mechanisms. Therefore, to better estimate the overall antioxidant activity of a sample, at least two (or even all) of these assays must be combined. Tan and Lim (2015) recommended a mix of single electron transfer based (DPPH, ABTS, FRAP, TBARS) and hydrogen atom transfer based (ORAC, HORAC, TRAP, crocin and $\beta$-carotene bleaching) assays. Among the six in vitro studies recorded in this review, just two (Prasad et al., 2013a; Prasad et al., 2013b) met with this requirement, while the other ones only used $\beta$-carotene bleaching (Mmbengwa et al., 2009) or DPPH (Awouafack et al., 2013b; Thongnest et al., 2013; Awouafack et al., 2018) assays. In vitro models are known to be not always reproducible in respective in vivo assays. Therefore, in vivo studies are necessary for the confirmation of such effects. Interestingly, the weakly active ethanol root extract of E. chinense, its chloroform fraction and the inactive compound 39 using DPPH method, restored the alterations of nitric oxide, TBARS, superoxide dismutase and catalase activities in the castor oil-mediated diarrhea model in rats (Prasad et al., 2013c). Although data at this level seems to be naïve and preliminary for any conclusion, they are indicator of the antioxidant potential of members of the Eriosema genus. However, more species need to be investigated in vitro and in vivo.

\section{Estrogenic and Aryl Hydrocarbon Agonistic Properties}

Estrogens deficiency (natural or surgical) is associated with numerous health problems including urogenital atrophy, hot flushes, osteoporosis and increased risk of cardiovascular diseases (Calleja-Agius and Brincat, 2015; Nappi and Cucinella, 2020). Given the adverse outcomes associated with the long-term usage of the hormone replacement therapy e.g. increased risk of endometrial and breast cancer, stroke and pulmonary thromboembolism, phytoestrogens have gained and continue to receive great interest.

The estrogenic properties of E. laurentii, traditionally used against female infertility, gynecological and menopausal complaints have been evaluated in vitro and in vivo. In vitro, a methanol extract of the aerial parts (AEL), its fractions and the predominant secondary metabolites 43,44 and 73 induced a significant and concentration-dependent $\beta$-galactosidase activity in the ERa yeast two-hybrid assay (yERa) (Ateba et al., 2013; Ateba et al., 2014b). At $25 \mu \mathrm{g} / \mathrm{ml}$, AEL was as potent as $10 \mathrm{nM}$ estradiol (Ateba et al., 2013). Compounds 43, 44 and 73 displayed $\mathrm{EC}_{50}$ values of $0.32 \mu \mathrm{M}, 6.1 \mu \mathrm{M}$ and $21.4 \mathrm{nM}$, respectively, while that of $17 \beta$-estradiol $\left(\mathrm{E}_{2}\right)$ was $0.27 \mathrm{nM}$. All the three compounds were full ERa agonists as they displayed the maximal efficacy of $E_{2}$ although at much higher concentrations (Ateba et al., 2014b). A methanolic extract of the underground parts of E. laurentii (UEL) was also highly active in yERa (Ateba et al., 2016b). The concentration of $10 \mu \mathrm{g} / \mathrm{ml}$ was as potent as $0.5 \mathrm{nM} E_{2}$. However, compounds 22, 62 and 63 from this extract did not show any yERa activity (Ateba et al., 2016b). 
In vivo, a 3-days uterotrophic assay and a 9-weeks oral treatment in ovariectomized adult rats were tested. AEL at the doses of 50,100 and $200 \mathrm{mg} / \mathrm{kg} \mathrm{p}$. o. did not induce endometrium proliferation either in the 3-days or the 9weeks treatment regimens, but induced vaginal stratification and cornification (Ateba et al., 2013). This tissue-dependent effect suggests that AEL could prevent vaginal dryness experienced by menopausal women, while not inducing the unwanted uterine proliferation. In the other hand, the decoction of aerial parts of E. laurentii had no significant effect on the uterine and vaginal endpoints at all tested doses following a 3days uterotrophic assay (Ateba et al., 2014c).

Studies reported the repressive effects of aryl hydrocarbon receptor (AhR) agonists on ERa estrogen-sensitive cancer (endometrium and breast) cells (Tsuchiya et al., 2005; Okino et al., 2009; Marconett et al., 2010; Labrecque et al., 2012). In line with this, the agonistic properties of AEL and compounds were evaluated. AEL exhibited a dose-dependent and significant AhR $\beta$-galactosidase activity from $5 \mu \mathrm{g} / \mathrm{ml}$. The activity at $83 \mu \mathrm{g} / \mathrm{ml}$ was similar to that of $50 \mathrm{nM} \beta$-naphthoflavone (Ateba et al., 2013). For the first time, 44 (EC 50 not determined) and $73\left(\mathrm{EC}_{50}\right.$ of $1.34 \mu \mathrm{M}$ ) were found as partial agonists of AhR, whereas 43 was not active (Ateba et al., 2014b).

Classical, suitable and reproducible systems/models have been used to evaluate estrogenic and aryl hydrocarbon agonistic properties of E. laurentii in vitro (Jungbauer and Beck, 2002; Reiter et al., 2009) and in vivo (OECD, 2007; Mvondo et al., 2011). Accordingly, all data reported in this section demonstrated the potential of E. laurentii for managing various gynecological and menopausal complaints. However, this species is also traditionally used for treating female infertility. Studies in that domain are needed. Similarly, a wide traditional use of $E$. cordatum in South Africa against female infertility has been recorded. Nevertheless, no published study demonstrates this potential till now.

\section{Antiosteoporosis Activity}

Osteoporosis is a skeletal disorder characterized by a deterioration of bone tissue and disruption of bone microarchitecture that lead to low bone mass and strength, predisposing to an increased risk of fracture (Pisani et al., 2016). It affects both sexes and is mainly linked with the agerelated decrease of estradiol and testosterone. Therapeutic agents that would not only inhibit bone resorption but also simultaneously stimulate bone formation would be most favorable (Abdelsamie et al., 2019).

Using a well-known postmenopausal-like model of osteoporosis (ovariectomy) in rats, Ateba et al. (2013) evaluated the effects of a 9-weeks oral treatment with a methanolic extract of the aerial parts of E. laurentii. The extract significantly increased the dry femur weight (at 50 and $200 \mathrm{mg} / \mathrm{kg}$ ), and calcium levels in serum (at 50 and $200 \mathrm{mg} / \mathrm{kg}$ ) and femur (at all tested doses) compared with ovariectomized animals. The serum levels of inorganic phosphorus decreased, while the femur levels increased in a dose-dependent manner. Although the dose of $200 \mathrm{mg} / \mathrm{kg}$ was not able to completely reverse the ovariectomy-increased alkaline phosphatase (a biomarker for osteoblastic activity and bone remodeling), it decreased it by $\sim 15 \%$. Based on the increased in femur mass, and femur calcium and inorganic phosphorus contents, the authors concluded that bone formation exceeded resorption suggesting the inhibition of bone resorption and activation of bone formation.

There is growing interest in $17 \beta$-hydroxysteroid dehydrogenase type 2 (17 $\beta$-HSD2), an enzyme able to regulate the intracellular concentration of estradiol and testosterone by transforming them into the less potent forms estrone and androstenedione (Marchais-Oberwinkler et al., 2011) in tissues where it is expressed, such as bone (Dong et al., 1998; Eyre et al., 1998). Blockade of this enzyme is thought to increase intracellular estradiol and testosterone, which thereby inhibits bone resorption by osteoclasts and stimulates bone formation by osteoblasts (Abdelsamie et al., 2019). In line with this 17 $\beta$-HSD2 is more and more considered as target for antiosteoporosis treatment. In an in vitro assay using lysates of cells expressing the recombinant human enzyme 17 $\beta$-HSD2, Vuorinen et al. (2017) tested 36 hit molecules, including $\mathbf{4 4}$ and $\mathbf{7 3}$ from E. laurentii. These compounds with respective $\mathrm{IC}_{50}$ values of 1.52 and $2.03 \mu \mathrm{M}$ were among the most active $17 \beta$-HSD2 inhibitors tested. However, they selectively inhibited $17 \beta$-HSD1 (that catalyzes the reverse reaction) over $17 \beta$-HSD2 and therefore cannot be suitable lead structures for a possible therapeutic use (Vuorinen et al., 2017).

\section{Erectile Dysfunction and Impotence}

Erectile dysfunction (ED) as well as impotence is a multifactorial (psychogenic, neurogenic, vasculogenic, endocrine/metabolic, end organ disease and iatrogenic) health issue common in $20-30 \%$ of adult men worldwide (McMahon, 2019; Taylor et al., 2019). It negatively impacts patient's sexual satisfaction and psychological well-being, leading to a greater prevalence of anxiety and depression. Lifestyle changes in combination with oral phosphodiesterase type 5 inhibitors (PDE5Is: sildenafil, tadalafil, vardenafil and avanafil) are typically first-line treatments of most etiologies of ED (Hatzimouratidis et al., 2016; Krzastek et al., 2019; Moses et al., 2019). PDE5Is increase arterial blood flow into the penis (via relaxion of the corpus cavernosum and deep penile artery smooth muscles) and reduce the venous outflow leading to the penile erection. Among PED5Is, only sildenafil is cost-effective as it is the only PDE5-I with a generic option. PED5Is are commonly associated with side effects (flushing, hypotension, headache, dyspepsia, back pain, myalgia, dizziness, blurred vision and rhinitis) and a lot of contraindications that contribute to significant treatment dropout rates (Yuan et al., 2013; Yafi et al., 2018). Moreover, up to $40 \%$ of ED patients fail to respond sufficiently to the maximum dose of PDE5Is (Porst et al., 2013).

From ancient times, plants have served as a dependable source of medicines for treating ED. In the Eriosema genus, E. cordatum, E. kraussianum and E. salignum are traditionally or locally used as aphrodisiac or against male sexual disorders (ED, impotence). However, only compounds from E. kraussianum were evaluated until now. In 2002, Drewes et al. investigated the capacity of 
pyrano-isoflavones 64-68 to relax the corpus cavernosum of rabbits. Compounds 64 and $\mathbf{6 5}$ displayed a dose-dependent activity, while 66-68 were inactive. At the low concentration of $78 \mathrm{ng} / \mathrm{ml}, 64$ and 65 displayed 85 and $65 \%$ of the relaxation found with sildenafil citrate, respectively. Rather to induce relaxation, $\mathbf{6 6}$ and $\mathbf{6 8}$ caused contraction of corpus cavernosum tissue (Drewes et al., 2004). Accordingly, the authors indicated that the overall effect of the extract as used in traditional medicine is mainly determined by the activities of 64 and 65 , present as the major constituents.

Eriosema cordatum, E. kraussianum and E. salignum and the other Eriosema species that come under the isiZulu indigenous umbrella name of "uBangalala" are widely traditionally used mainly in South Africa for curing or alleviating ED and/or impotence (Bryant, 1966; Hutchings et al., 1996). However, despite their high reputation in that domain, the investigation only focused on E. kraussianum with two papers published more than 15 years ago. In line with this, these Eriosema species traditionally used against ED remain underexplored and even seem to be abandoned. Given the burden of this sensitive topic, they require more research attention.

\section{Hypoglycemic and Anti-diabetic Effects}

Diabetes is a major global health threat. This metabolic disorder is caused by a lack (absolute or relative) of insulin and/or resistance to insulin that lead to chronic hyperglycemia.

ED is a common comorbidity of diabetes mellitus. It can be caused by impaired hemodynamic mechanisms in the penile and ischemic vasculature that occur in diabetes mellitus (Rendell et al., 1999). There is a consensus that lifestyle changes and risk factor modification must precede or accompany any pharmacological or psychological ED treatment (Hatzimouratidis et al., 2016; Hackett et al., 2018). In other words, the amelioration of diabetes risk factors may improve erectile function. Acute oral treatment with a E. kraussianum rootstock hydro-alcoholic extract (40-320 mg/kg) dosedependently and significantly reduced fasting blood glucose levels in normoglycemic and streptozotocin-treated diabetic rats from 2 to $8 \mathrm{~h}$ after treatment with peak effects after $4 \mathrm{~h}$ (Ojewole et al., 2007). Using an oral glucose tolerance test, a pretreatment with the extract $20 \mathrm{~min}$ before challenge significantly reduced the peak blood glucose levels. Similar effects were obtained with $80 \mathrm{mg} / \mathrm{kg}$ p. $o$. of kraussianones 1 and 2 in normoglycemic rats (Ojewole et al., 2006).

In alloxan-induced diabetic Wistar rats, both aqueous and ethanolic leaf extracts of E. psoraleoides (at 200 and $400 \mathrm{mg} / \mathrm{kg}$ ) significantly decreased fasting blood glucose levels from day 6 of a 7-days treatment (Nduka et al., 2018; Nduka et al., 2019). Using the same model, Elechi et al. (2019) investigated the effects of $n$-hexane, $n$-hexane/dichloromethane $(1: 1 \mathrm{v} / \mathrm{v})$, dichloromethane, and dichloromethane/methanol (19:1 v/v) fractions of the $n$-hexane leaf extract of $E$. psoraleoides on the fasting blood glucose levels. The $n$-hexane/ dichloromethane and the dichloromethane fractions at the dose of $200 \mathrm{mg} / \mathrm{kg}$ produced a significant decrease in fasting blood glucose levels from day 2-7, the effects of the dichloromethane fraction being higher or very close to that of $4 \mathrm{mg} / \mathrm{kg}$ glibenclamide (Elechi et al., 2019).
Eriosema glomeratum claimed to be traditionally used against diabetes (Lawin et al., 2015) is not yet investigated in relation to the metabolic disorder.

\section{Cardiovascular protection: dyslipidemia, vasodilatation and preeclampsia}

Cardiovascular disease remains the leading cause of death worldwide (Global Burden of Disease Study 2017; Causes of Death Collaborators, 2018). Managing its modifiable risk factors such as hypertension and dyslipidemia constitutes key targets of public health organizations over the world (Berman et al., 2019; Michos et al., 2019).

Increased risk of cardiovascular diseases such dyslipidemia is well known to be associated with menopause. Using a postmenopause-like model (ovariectomized Wistar rats), a 9weeks oral treatment with a methanol extract of the aerial parts of E. laurentii significantly decreased the total cholesterol (TC) and LDL levels at $200 \mathrm{mg} / \mathrm{kg}$. Increased HDL as well as a nonsignificant decrease of triglycerides (TG) levels were also observed at all tested doses (50, 100 and $200 \mathrm{mg} / \mathrm{kg}$ ). The extract decreased the ovariectomy-increased TC/HDL and LDL/HDL ratios. A significant decrease of the atherogenic plasma index [AIP: $\log _{10}$ (TG/HDL)] was determined at all tested doses (Ateba et al., 2013). Individual cholesterol risk factors are not sufficient to assess cardiovascular risk (Michos et al., 2019). A huge body of evidence supports AIP as one of the strongest predictive indicator of cardiovascular disease risk (Dobiášová et al., 2004; Edwards et al., 2017) also in postmenopausal women (Wu et al., 2018; Barua et al., 2019). This parameter has been evaluated in the study by Ateba et al. (2013) indicating the potential of E. laurentii in the management of dyslipidemia in postmenopausal women.

Ojewole et al. (2006) investigated the vasodilatory effects of 64 and $65(100-2,000 \mu \mathrm{g} / \mathrm{ml})$ from the rootstock of E. kraussianum in isolated portal veins. Both constituents, after provoked initial slight contraction, induced a dose-dependent secondary and pronounced vasorelaxant effect, probably by affecting calcium mobilization and/or sequestration, and possibly also, calcium release from its various tissue stores.

Preeclampsia is the most common pregnancy-related complication worldwide, affecting $5-7 \%$ of all pregnancies with estimated 70,000 maternal deaths and 500,000 fetal and neonatal deaths each year (Rana et al., 2019). This incidence probably remains underestimated due to underreporting (Mayrink et al., 2018). The options for prevention and treatment of this hypertensive disorder are extremely limited, especially in resource-limited settings (Lemoine and Thadhani, 2019) where almost all of the deaths occur (Oyston and Baker, 2020). In this context, medicinal plants and herbs are an opportunity. In preeclampsia, the abnormal placentation characterized by impaired spiral artery remodeling lead to an ischemic placenta, which releases factors such as anti-angiogenic factors sFlt-1 (soluble fms-like tyrosine kinase-1) and sEng (soluble Endoglin), responsible for vascular dysfunction, into the maternal circulation (Chang et al., 2018; Eddy et al., 2018; Jena et al., 2020). Based on the vasodilatory properties of $\mathbf{6 5}$ 
(Drewes et al., 2002; Ojewole et al., 2006), Ramesar et al. (2012) investigated its effects in the L-NAME-induced preeclamptic Sprague-Dawley rat model. Subcutaneously administered at $10 \mathrm{mg} / \mathrm{kg}$ for 12 consecutive days, the compound decreased fetal mortality, demonstrated a trend toward increasing birth and placental weights by improving placental perfusion, reducing blood pressure amplification through a NO-independent mechanism, and decreasing the plasma levels of sFlt-1and sEng.

Globally, based on the in vivo models used and the results obtained, the investigated Eriosema species display a potential in the management of modifiable risk factors (hypertension, dyslipidemia) of the cardiovascular disease.

\section{Acetylcholinesterase Inhibition}

The inflammation-associated cognitive decline is mainly due to degeneration of central cholinergic neurons or cholinergic impairment, making acetylcholinesterase inhibitors the main class of drugs currently used in the treatment of mild cognitive impairment and Alzheimer's disease (Benfante et al., 2019). In a study by Ndhlala et al. (2011), an aqueous extract of the roots of E. cordatum with an $\mathrm{IC}_{50}$ value of $756.6 \mu \mathrm{g} / \mathrm{ml}$ showed a very low acetyl cholinesterase inhibitory activity.

\section{Anti-Microbial Activity}

Infectious diseases are among the most important cause of morbidity and mortality worldwide. This is worsened by drug resistance, one of the greatest challenges of the 21st century (Hofer, 2019; Tacconelli and Pezzani, 2019). The importance of plants as a source of effective anti-microbial agents is well established. Several reports dealing with the anti-bacterial and anti-fungal effects of Eriosema species have been documented.

Flavonoids from the roots of $E$. chinense were tested against Gram-negative (Escherichia coli 25,922, Klebsiella pneumoniae and Pseudomonas aeruginosa) and Gram-positive (Bacillus cereus, Enterococcus faecalis, Staphylococcus aureus, methicillinresistant S. aureus (S. aureus MRSA), Staphylococcus epidermidis, Streptococcus agalactiae, Streptococcus pyogenes and Listeria monocytogenes) bacteria, and Candida albicans using agar dilution technique. Compound $\mathbf{2 7}$ displayed a strong activity (minimum inhibitory concentration (MIC) of $2.3 \mu \mathrm{g} / \mathrm{ml}$ ) against S. agalactiae and S. pyogenes. MIC values of $2.3 \mu \mathrm{g} / \mathrm{ml}$ (toward S. pyogenes), $4.7 \mu \mathrm{g} / \mathrm{ml}$ (toward B. cereus and $S$. agalactiae) and $9.4 \mu \mathrm{g} / \mathrm{ml}$ (toward L. monocytogenes, S. aureus MRSA, S. epidermidis) were observed with compound 23. Compound 40 displayed MIC values of $4.7 \mu \mathrm{g} / \mathrm{ml}$ toward $B$. cereus, E. faecalis, L. monocytogenes, and all Streptococcus and Staphylococcus strains. Compound $\mathbf{2 5}$ significantly inhibited the growth of B. cereus (MIC of $2.3 \mu \mathrm{g} / \mathrm{ml}$ ), S. pyogenes (MIC of $2.3 \mu \mathrm{g} / \mathrm{ml}$ ), S. aureus (MIC of $4.7 \mu \mathrm{g} / \mathrm{ml}$ ), S. aureus MRSA (MIC of $4.7 \mu \mathrm{g} / \mathrm{ml}$ ), S. agalactiae (MIC of $4.7 \mu \mathrm{g} / \mathrm{ml}$ ), and E. faecalis (MIC of $9.4 \mu \mathrm{g} / \mathrm{ml}$ ) and L. monocytogenes (MIC of $9.4 \mu \mathrm{g} / \mathrm{ml}$ ). With 20 , a MIC value of $9.4 \mu \mathrm{g} / \mathrm{ml}$ was obtained against B. cereus, S. aureus, S. aureus MRSA, S. agalactiae, S. epidermidis and S. pyogenes, and of $18.8 \mu \mathrm{g} / \mathrm{ml}$ toward E. faecalis and L. monocytogenes. The standard amphotericin B resulted in a MIC of $0.12 \mu \mathrm{g} / \mathrm{ml}$ against $C$. albicans, while chloramphenicol displayed MIC values of $10 \mu \mathrm{g} / \mathrm{ml}$ against $E$. coli $25,922, K$. pneumoniae, $B$. cereus, E. faecalis, L. monocytogenes, S. aureus and S. aureus MRSA, and of $1 \mu \mathrm{g} / \mathrm{ml}$ toward S. agalactiae, S. epidermidis and $S$. pyogenes (Thongnest et al., 2013).

Dichloromethane and ethanolic root extracts of E. cordatum exhibited moderate antibacterial activities against $S$. aureus, $B$. subtilis, E. coli and K. pneumoniae (Ndhlala et al., 2011). The ethanol extract showed MIC values of $390 \mu \mathrm{g} / \mathrm{ml}$ toward all these microbial species, while that of the dichloromethane extract were $195 \mu \mathrm{g} / \mathrm{ml}$ against S. aureus and B. subtilis, and $390 \mu \mathrm{g} / \mathrm{ml}$ against E. coli and K. pneumoniae. Petroleum ether and water root extracts were not active (MIC $\geq 1.56 \mathrm{mg} / \mathrm{ml}$ ) against all the tested microbial species. The MIC of the standard neomycin ranged between $0.8 \mu \mathrm{g} / \mathrm{ml}$ and $1.6 \mu \mathrm{g} / \mathrm{ml}$ (Ndhlala et al., 2011).

The ethanolic root extract of E. chinense (Prasad et al., 2013c) as well as its hexane, chloroform and ethyl acetate fractions (Prasad et al., 2013b) displayed MIC $>195 \mu \mathrm{g} / \mathrm{ml}$ against reference bacterial strains E. coli ATCC25922, Shigella flexneri ATCC12022, P. aeruginosa ATCC27893, S. aureus ATCC25323 and clinical isolates of Salmonella typhi, Shigella dysenteriae, Proteus vulgaris, K. pneumoniae, Shigella boydii, B. cereus and E. faecalis. In these studies, IZ values $(24.1-30.7 \mathrm{~mm})$ of the standard ciprofloxacin $(0.5 \mathrm{mg} / \mathrm{ml})$ were determined, but MIC values not stated. Compounds 18, 34, 39 and $\mathbf{4 0}$ from the roots of E. chinense showed MIC values of $12.5 \mu \mathrm{g} / \mathrm{ml}$ against Mycobacterium tuberculosis $\mathrm{H} 37 \mathrm{Ra}$, while that of $\mathbf{2 3}, 27$ and 38 were $25 \mu \mathrm{g} / \mathrm{ml}$. Those of the standards isoniazid and streptomycin were $0.023-0.046$ and $0.156-0.313 \mu \mathrm{g} / \mathrm{ml}$, respectively (Sutthivaiyakit et al., 2009).

Using the disk diffusion method, the essential oil from the fresh leaves of E. englerianum (1, 2, 5 and $10 \mu \mathrm{L} / \mathrm{ml}$ ) was tested against eight different bacteria ( $S$. aureus, E. coli, $P$. aeruginosa, Bacillus subtilis, K. pneumoniae, P. vulgaris, Clostridium sporogenes and Acinetobacter calcoaceticus). Considered as positive result by authors, a diameter of inhibition zone (IZ) $\geq$ $7 \mathrm{~mm}$ was observed at $5 \mu \mathrm{L} / \mathrm{ml}$ against $A$. calcoaceticus, and at $10 \mu \mathrm{L} / \mathrm{ml}$ against $C$. sporogenes and A. calcoaceticus (Mmbengwa et al., 2009). Against opportunistic fungi, a concentrationdependent inhibition of C. albicans (24.2-42.6\%), Aspergillus niger (34.7-49.7\%) and Aspergillus flavus (27.5-39.3\%) at concentrations of $1-10 \mu \mathrm{L} / \mathrm{ml}$ was seen, while nystatin inhibited them by $84.3,76.3$ and $76.3 \%$, respectively. However, the concentration of nystatin displaying this activity was not indicated (Mmbengwa et al., 2009).

Through the broth dilution method, methanol/ dichloromethane $(1: 1, \mathrm{v} / \mathrm{v})$ extracts of stems and leaves of $E$. glomeratum showed MIC values ranging between 50 and $1000 \mu \mathrm{g} / \mathrm{ml}$ against S. aureus, K. pneumoniae, Morexella catarrhalis, Mycobacterium smegmatis and Mycobacterium aurum (Fomogne-Fodjoet al., 2014). The stem extract displayed the highest activity with MIC values of 65 and $50 \mu \mathrm{g} / \mathrm{ml}$ against $M$. smegmatis and $M$. aurum, respectively, whereas the leaf extract was most active against $M$. smegmatis (MIC $130 \mu \mathrm{g} / \mathrm{ml}$ ). Against these two Mycobacterium species, the standard ciprofloxacin displayed MIC values of 78 and $1.5 \mathrm{ng} / \mathrm{ml}$, respectively. Compounds 1, 2, 13, 47 and 85 from the methanol/ dichloromethane $(1: 1 \mathrm{v} / \mathrm{v})$ extract of the whole plant of E. glomeratum were tested at a concentration of $1 \mu \mathrm{g} / \mathrm{ml}$ 
against bacteria (Bacillus megaterium, E. coli), the green alga Chlorella fusca and the fungus Microbotryum violaceum using an agar diffusion assay (Awouafack et al., 2008). Compound 1 displayed IZ of $7 \mathrm{~mm}$ toward B. megaterium, C. fusca and $M$. violaceum, and of $10 \mathrm{~mm}$ against $E$. coli. IZ values of $10,8,9$ and $13 \mathrm{~mm}$ were observed with 2 against B. megaterium, E. coli, $C$. fusca and M. violaceum, respectively. Compounds 13 and 47 were only active (IZ of $10 \mathrm{~mm}$ ) against $C$. fusca, while 85 was inactive against all four microbial species. In this study, the standards were also tested at a concentration of $1 \mu \mathrm{g} / \mathrm{ml}$. Tetracycline displayed IZ of $18 \mathrm{~mm}$ against bacteria, while an IZ of $20 \mathrm{~mm}$ was observed with nystatin against $M$. violaceum. The IZ of the standard actidione was 35 and $50 \mathrm{~mm}$ against the alga and fungus, respectively (Awouafack et al., 2008).

A polar fraction of an ethanolic leaf extract of E. montanum displayed a low antimicrobial activity with a MIC $>750 \mu \mathrm{g} / \mathrm{ml}$ against bacteria (B. cereus ATCC 14579, Enterobacter cloacae ATCC 13047, E. coli ATCC 8739, K. pneumoniae ATCC 13883, Mycobacterium fortuitum ATCC 6841, P. vulgaris ATCC 13315, P. aeruginosa ATCC 15442, Salmonella typhimurium ATCC 13311, S. aureus ATCC 6538, Streptococcus pyogenes ATCC 12344) and fungi (C. albicans ATCC 10231, isolates of Trichophyton rubrum RV 58125, Epidermophyton floccosum RV 71625 and Microsporum canis RV 66973) (Cos et al., 2002b).

Methanolic extracts of the bark and wood of twigs of $E$. psoraleoides displayed very high MIC values $(\geq 1.25 \mathrm{mg} / \mathrm{ml})$ against Streptococcus mutans HG 982, Actinomyces viscosus HG485 and C. albicans HG392 in the agar dilution method (Khan et al., 2000). MIC values of 8,32 and $128 \mu \mathrm{g} / \mathrm{ml}$ against these microorganisms were observed with the standard chlorhexidine, respectively. Using a bioautography agar overlay method, Runyoro et al. (2006) reported that a methanolic extract of the stem bark of E. psoraleoides was not active against $C$. albicans. A methanolic leaf extract as well as its $n$-hexane and ethyl acetate fractions were tested against E. coli ATCC35219, S. aureus ATCC25923 and clinical isolates of $S$. aureus, $P$. vulgaris, Klebsiella aerogenes, P. aeruginosa, and E. coli and displayed MIC $>1.26 \mathrm{mg} / \mathrm{ml}$, except the $n$-hexane fraction with a MIC value of $150 \mu \mathrm{g} / \mathrm{ml}$ toward S. aureus ATCC. The MIC value of the standard gentamicin was not reported (Elechi and Igboh, 2017).

An ethanolic extract from the twigs of E. robustum showed a MIC value of $80 \mu \mathrm{g} / \mathrm{ml}$ against S. aureus, E. faecalis and E. coli and of $310 \mu \mathrm{g} / \mathrm{ml}$ toward $P$. aeruginosa. Significant differences in the activity were observed against the fungi Aspergillus fumigatus (MIC $160 \mu \mathrm{g} / \mathrm{ml}$ ), Cryptococcus neoformans (MIC $630 \mu \mathrm{g} / \mathrm{ml}$ ), C. albicans and C. albicans ATCC (MIC 1,250 $\mu \mathrm{g} / \mathrm{ml}$ ) (Awouafack et al., 2013a). Compounds from this extract showed either moderate or low activity against these microbial species. Moderate antimicrobial activities (MIC 63 or $65 \mu \mathrm{g} / \mathrm{ml}$ ) were observed with 16 and 17 against C. albicans ATCC, A. fumigatus and $P$. aeruginosa. 17 also showed a moderate activity against Cryptococcus neoformans and E. faecalis. A MIC of 63 or $65 \mu \mathrm{g} /$ ml was observed with 107 against $P$. aeruginosa and with 87 against A. fumigatus and $P$. aeruginosa; 101 against $C$. albicans ATCC and $P$. eruginosa; 99 against $S$. aureus and $P$. aeruginosa; 88 against A. fumigatus, $C$. neoformans, $P$. aeruginosa and E. faecalis; $\mathbf{4 1}$ against C. albicans ATCC, S. aureus, P. aeruginosa, E. faecalis and E. coli
(Awouafack et al., 2013a). The standard gentamicin showed a MIC $<3.91 \mu \mathrm{g} / \mathrm{ml}$ against bacteria, while that of amphotericin B was $30 \mu \mathrm{g} / \mathrm{ml}$ toward C. albicans (isolate) and C. albicans (ATCC), $>250 \mu \mathrm{g} / \mathrm{ml}$ against C. neoformans and $125 \mu \mathrm{g} / \mathrm{ml}$ against $A$. fumigatus. Using a microdilution method, $\mathbf{5}$ and $\mathbf{7 2}$ from the ethanol extract of twigs of E. robustum displayed weak antimicrobial activity (MICs $>150 \mu \mathrm{g} / \mathrm{ml}$ ) against Bacillus subtilis, S. aureus, K. pneumoniae, E. coli, C. albicans, and Saccharomyces cerevisiae (Awouafack et al., 2018) In this study, the tested concentrations or MIC values of standard antibiotics ampicillin, kanamycin, and cycloheximide were not indicated.

Using the broth microdilution method, the antifungal activity of methanolic leaf extracts $(500 \mu \mathrm{g} / \mathrm{ml})$ of Eriosema campestre var. campestre, Eriosema campestre var. macrophyllum, Eriosema glabrum Mart. ex Benth., Eriosema heterophyllum Benth., Eriosema longifolium Benth. and Eriosema tacuaremboense Arechav. was evaluated. After $72 \mathrm{~h}$ incubation, $E$. heterophyllum was fungistatic against T. rubrum, Trichophyton mentagrophytes and Microsporum gypseum and fungicidal against Epidermophyton floccosum. The extracts of E. campestre var. macrophyllum and E. glabrum, respectively, displayed fungistatic and fungicidal activities against T. mentagrophytes and E. floccosum. The extract of E. campestre var. campestre was fungistatic toward T. rubrum, T. mentagrophytes and E. floccosum. Moreover, all these Eriosema species were not active against C. albicans, Candida krusei, Candida glabrata, Candida tropicalis and Candida parapsilosis after $48 \mathrm{~h}$ incubation (de Morais et al., 2017).

The bioassay-guided fractionation of a dichloromethane root extract of E. tuberosum using a TLC bioautography assay led to the isolation of compounds 34-36, 40, 62 and 75. Amounts of $5 \mu \mathrm{g}$ of $35,36,40$ and 62 , and $10 \mu \mathrm{g}$ of $\mathbf{3 4}$ on TLC plates prevented the growth of Cladosporium cucumerinum, while $1 \mu \mathrm{g}$ of 34,36 , 40 and 62, and $5 \mu \mathrm{g}$ of 35 inhibited the growth of Candida albicans. Eriosematin (75) was not active against C. cucumerinum and C. albicans (Ma et al., 1995). 77 from the same extract inhibited the growth of both fungi at $2.5 \mu$ g, while 76, 78 and 79 were not active (Ma et al., 1996a). Compounds 80 and 81 exhibited a strong activity (inhibition at $0.5 \mu \mathrm{g}$ ) against $C$. cucumerinum and a weak activity (inhibition at $30 \mu \mathrm{g}$ ) against C. albicans (Ma et al., 1996b). Moreover, 10 and $30 \mu \mathrm{g}$ of 39 were active against $C$. cucumerinum and C. albicans, respectively. In the same assay, $2 \mu \mathrm{g}$ of 98 and $3 \mu \mathrm{g}$, each, of 94, 96 and 104 inhibited the growth of C. cucumerinum, 82, 105 and 106 were not active against C. cucumerinum (Ma et al., 1999).

In a study by Sianglum et al. (2019), lupinifolin (39) demonstrated an antimicrobial activity against $E$. faecalis ATCC29212, S. aureus ATCC25923 as well as susceptible and multidrug-resistant enterococcal clinical isolates (E. faecalis and E. faecium) in the standard broth microdilution method. Although the tested compound was not extracted from an Eriosema species, it has been reported in roots of E. chinense and E. robustum. In that study, 39 displayed activity against all susceptible and resistant strains with MICs and MBCs (minimum bactericidal concentration) ranging between 0.5 and $2 \mu \mathrm{g} / \mathrm{ml}$ and between 2 and $16 \mu \mathrm{g} / \mathrm{ml}$, respectively. Toward all the strains, compound 39 with MIC values of $0.5-2 \mu \mathrm{g} / \mathrm{ml}$ was more active 
than vancomycin (MIC of $1-256 \mu \mathrm{g} / \mathrm{ml}$ ). It increased membrane permeability and caused loss of salt tolerance. On E. faecalis ATCC29212, E. faecalis HTY0037 (a multidrug resistance isolate) and E. faecium HTY0256 (a strong biofilm-producing vancomycin-resistant enterococci isolate), after $2 \mathrm{~h}$ incubation it displayed significant rapid antibacterial activity. Moreover, an antibiofilm-producing activity of 39 against four MDR enterococci was shown.

Eighteen articles dealing with the antimicrobial activity of Eriosema species have been published using disk diffusion, broth dilution and thin layer chromatography (TLC) bioautography methods. The "traditional" inhibitory zone (IZ) determined by the disc diffusion method depends on the coefficient of diffusion of compounds, highly polar substances diffusing more easily in agar and displaying a high IZ and vice versa (Tan and Lim, 2015). Therefore, this assay is only useful for a simple qualitative screening as it does not allow the determination of the real amount of the antimicrobial agent that diffuses into the agar, impeding the determination of MICs and MBCs (Balouiri et al., 2016). Based on the MIC values, extracts (or compounds) display: i) a strong antimicrobial activity in vitro if the $\mathrm{MIC} \leq 100 \mu \mathrm{g} / \mathrm{ml}$ (or $10 \mu \mathrm{g} / \mathrm{ml}$ ), ii) moderate if $100<\mathrm{MIC} \leq 625 \mu \mathrm{g} / \mathrm{ml}$ (or $10<$ MIC $\leq 100 \mu \mathrm{g} / \mathrm{ml})$, iii) and low if MIC $>625 \mu \mathrm{g} / \mathrm{ml}$ (or $>100 \mu \mathrm{g} /$ ml) (Eloff, 2004; Ríos and Recio, 2005; Kuete, 2010). Based on this classification, several extracts and compounds from Eriosema species displayed promising antimicrobial activity. Unfortunately, in several studies positive controls were not included or their concentration was not stated. Although some studies are suggesting the potential of few compounds to act as an antimicrobial drug against several microorganisms, these deficiencies weaken the evidence.

\section{Anti-Viral Activity}

Cos et al. (2002a) assessed the anti-human immunodeficiency virus type-1 (HIV-1) effects of a polar fraction of an ethanolic leaf extract of Eriosema montanum Baker f. using a tetrazolium-based colorimetric assay in infected MT- 4 cells. The polar fraction was obtained suspending the extract in $60 \%$ methanol and defatting with petroleum ether. The polar fraction with an $\mathrm{EC}_{50}$ value $>166 \mu \mathrm{g} / \mathrm{ml}$ did not protect the HIV-infected cells.

At the maximal non-toxic concentrations of $375 \mu \mathrm{g} / \mathrm{ml}$, this polar fraction exhibited a pronounced antiviral activity (reduction factor of the viral titer (RF) of $10^{3}-10^{4}$ ) against Herpes simplex virus type 1 , Poliomyelitis virus type 1 strain 1A/S3 and Semliki forest virus A7, while at $187.5 \mu \mathrm{g} / \mathrm{ml}$ it displayed a RF of $10^{3}$ against Coxsackie B2 virus. RF values of $10^{2}$ were observed against Vesicular stomatitis virus T2 and measles Edmonston A at 187.5 and $750 \mu \mathrm{g} / \mathrm{ml}$, respectively (Cos et al., 2002b).

\section{Anti-Diarrheal Activity}

Diarrheal diseases are a leading cause of mortality and morbidity worldwide, mainly among children under-five in low developing countries. Defined as the discharge of 4 or more semisolid or watery feces per day, diarrhea involves an increase in intestinal fluid volume, in the frequency of bowel movement, wet stool and abdominal cramps, leading to loss of electrolytes and water. Its treatment and management depend on the duration and specific etiology which can be infectious or not. Over the world, many people or communities still use traditional herbs to treat a variety of diseases including diarrhea. As depicted in Table 1, several Eriosema species are used traditionally for diarrhea.

Castor oil-induced diarrhea is known as an efficient model for the initial screening for antimotility, antisecretory and antiinflammatory compounds. In the gut, ricinoleic acid (castor oil metabolite) causes irritation and inflammation in bowels leading to diarrhea (stomach cramp, increased peristaltic activity and intestinal fluid volume) (Matias et al., 1978; Racusen and Binder, 1979). An ethanol root extract of $E$. chinense (EEC; at $400 \mathrm{mg} / \mathrm{kg}$ ), its chloroform fraction (at $100 \mathrm{mg} / \mathrm{kg}$; CEC), and compounds 39 and $\mathbf{8 1}$ (at $10 \mathrm{mg} / \mathrm{kg}$ ) reduced the normal fecal excretion rate and water content 3,5 and $7 \mathrm{~h}$ after the oral treatment of rats. In castor oil-induced diarrhea, these treatments delayed the onset of diarrhea and decreased the mean defecation in a dosedependent manner. At the same dosages, the extract, the fraction and $\mathbf{3 9}$ and $\mathbf{8 1}$ significantly reduced the peristaltic index, the intestinal fluid volume and PGE2-induced enteropooling, and demonstrated a significant recovery from intestinal fluid loss of $\mathrm{Na}^{+}$and $\mathrm{K}^{+}$(Prasad et al., 2013c; Prasad et al., 2017).

Enteropathogenic E. coli (EPEC) is a common cause of moderate to severe water diarrhea in children, accompanied with fever, vomiting and a high hazard of death. In an enteropathogenic E. coli-induced diarrhea rat model, EEC (100 and $200 \mathrm{mg} / \mathrm{kg}$ ), CEC (50 and $100 \mathrm{mg} / \mathrm{kg}$ ) and 81 (5 and $10 \mathrm{mg} / \mathrm{kg}$ ) induced a significant recovery from diarrhea characterized by the reduction in total number of stools, total number of diarrheal stools, weight of stools, mean defecation rate and water content of stools $6 \mathrm{~h}$ after induction of diarrhea (Parmar et al., 2019a; Parmar et al., 2019b). At the higher tested doses, EEC, CEC and eriosematin E displayed diarrhea scores very close to that of $5.7 \mathrm{mg} / \mathrm{kg}$ norfloxacin. They also increased $\mathrm{Na}^{+} / \mathrm{K}^{+}$-ATPase activity to a higher level than that observed in the normal rats, resulting in reduced intestinal secretion.

Both infectious and non-infectious (chemically induced) reliable models were used to evaluate the anti-diarrheal potential of Eriosema species in vivo. However, from five species traditionally claimed to be anti-diarrheal (E. affine, E. chinense, E. griseum, E. psoraleoides and E. tuberosum), only the ethanolic root extract of $E$. chinense and compounds 39 and 81 were investigated, indicating that in the Eriosema genus this domain is underexplored. Given the promising results obtained with one extract, it would be interesting to extend the exploration to the other Eriosema species traditionally used against diarrhea.

\section{Anti-Inflammatory Activity}

Intestinal disorders often co-occur with inflammation and dysmotility. In enteropathogenic $E$. coli-induced diarrhea, $10 \mathrm{mg} / \mathrm{kg}$ of $\mathbf{8 1}$ from roots of E. chinense for $24 \mathrm{~h}$ significantly decreased the expression of pro-inflammatory cytokines IL-1 $\beta$ and TNF- $\alpha$ in colonic tissues (Parmar et al., 2019b). 
Using peripheral blood mononuclear cells, Santos et al. (2016) investigated the impact of a dichloromethane-ethanol extract of E. campestre $(6.25,12.5$ and $25 \mu \mathrm{g} / \mathrm{ml})$ on pathological processes of chronic inflammatory diseases. In a concentration-dependent manner, the extract inhibited the proliferation of T lymphocytes, including $\mathrm{CD}^{+}$and $\mathrm{CD}^{+}$cells, and decreased IL-2 levels in the supernatant of the cell cultures, a cytokine essential for the expansion of $\mathrm{T}$ lymphocytes.

Cyclooxygenase (COX) inhibitors are known to be a therapeutic target in inflammatory and neuroinflammatory diseases (Grosser et al., 2017; Dhir, 2019). The COX-1 and COX-2 inhibitory activities of extracts of the roots of $E$. cordatum were investigated by Ndhlala et al. (2011). At $250 \mu \mathrm{g} / \mathrm{ml}$, petroleum ether, dichloromethane and ethanolic extracts weakly inhibited COX-1 activity by 13,25 and $34 \%$, respectively. A weak inhibition of COX-2 by 6,35 and $10 \%$, respectively, with $250 \mu \mathrm{g} / \mathrm{ml}$ of the petroleum ether, dichloromethane and ethanolic extracts was observed. The aqueous extract remained almost without effects on both enzymes at $2 \mathrm{mg} / \mathrm{ml}$.

\section{Anthelmintic Activity}

Among human helminth infections, schistosomiasis is the most prevalent with regard to mortality and the third most harmful tropical disease in the world. An ethanol leaf extract of E. griseum was tested in vitro against trematodes (Echinostoma caproni and Schistosoma mansoni) and nematodes (Ancylostom aceylanicum, Heligmosomoides bakeri and Trichuris muris). The extract reduced the motility of third-stage larvae (L3) of $H$. bakeri by at least $80 \%$ at a minimal lethal concentration (MLC) of $20 \mu \mathrm{g} / \mathrm{ml}$ $48 \mathrm{~h}$ post-incubation, caused death of newly transformed $S$. mansoni schistosomula and adults with MLC values of 20 and $40 \mu \mathrm{g} / \mathrm{ml}$, respectively, exhibited a moderate activity against L3 of A. ceylanicum $48 \mathrm{~h}$ post-incubation and no activity on adult $E$. caproni and T. muris (MLC: $2 \mathrm{mg} / \mathrm{ml}$ ). In vivo, mice harboring adult S. mansoni, E. caproni and T. muris were used. An oral single dose of the extract $(400 \mathrm{mg} / \mathrm{kg}) 49$ days post-infection displayed a moderate activity in chronic S. mansoni infection (reduction of total worm burden $49.5 \%$ and of female worm burden $48.9 \%$ ), whereas no activity was observed against $E$. caproni and T. muris at 400 and $800 \mathrm{mg} / \mathrm{kg}$, respectively (Koné et al., 2012).

\section{TOXICOLOGICAL EVALUATION}

Although medicinal plants are widely used and assumed to be safe, however, they can potentially be toxic (Nasri and Shirzad, 2013; Awounfack et al., 2016). "If herbs have an effect, they are also likely to have a side effect" (Lanini et al., 2012). In line with this, the safety evaluation of medicinal plants-even used since centuries-needs to be performed. In vitro and in vivo studies have been carried out aiming at the evaluation of the safety properties of some Eriosema species.

In the Ames test on Salmonella typhimurium strain TA98 with and without S9 metabolic activation, a water extract of the roots of E. cordatum at concentrations up to $5,000 \mu \mathrm{g} / \mathrm{ml}$ was non-mutagenic as shown by the average $\mathrm{His}^{+}$revertant colonies (Ndhlala et al., 2011).

According to Koné et al. (2012), an ethanolic leaf extract of $E$. griseum displayed no toxicity against L6 rat skeletal myoblast cells. Awouafack et al. (2013a), evaluated the cytotoxicity of an ethanolic twig extract of E. robustum and compounds thereof against normal monkey Vero cells. In the MTT assay, the extract showed a low cytotoxicity ( $\mathrm{IC}_{50}$ of $53.45 \mu \mathrm{g} / \mathrm{ml}$ ). Among isolates obtained from this extract, 88 and 107 were not cytotoxic, 17, 41, 87 and 101 displayed a low cytotoxicity $\left(21.87 \leq \mathrm{IC}_{50} \leq 91.52 \mu \mathrm{g} / \mathrm{ml}\right)$, while 16 was moderately cytotoxic ( $\mathrm{IC}_{50}$ of $\left.13.20 \mu \mathrm{g} / \mathrm{ml}\right)$.

In vivo, the acute oral toxicity of an ethanol root extract (EEC) of $E$. chinense and its chloroform fraction (CEC) was evaluated in female rats following the OECD (Organization for Economic Cooperation and Development) guideline 425. Administration of

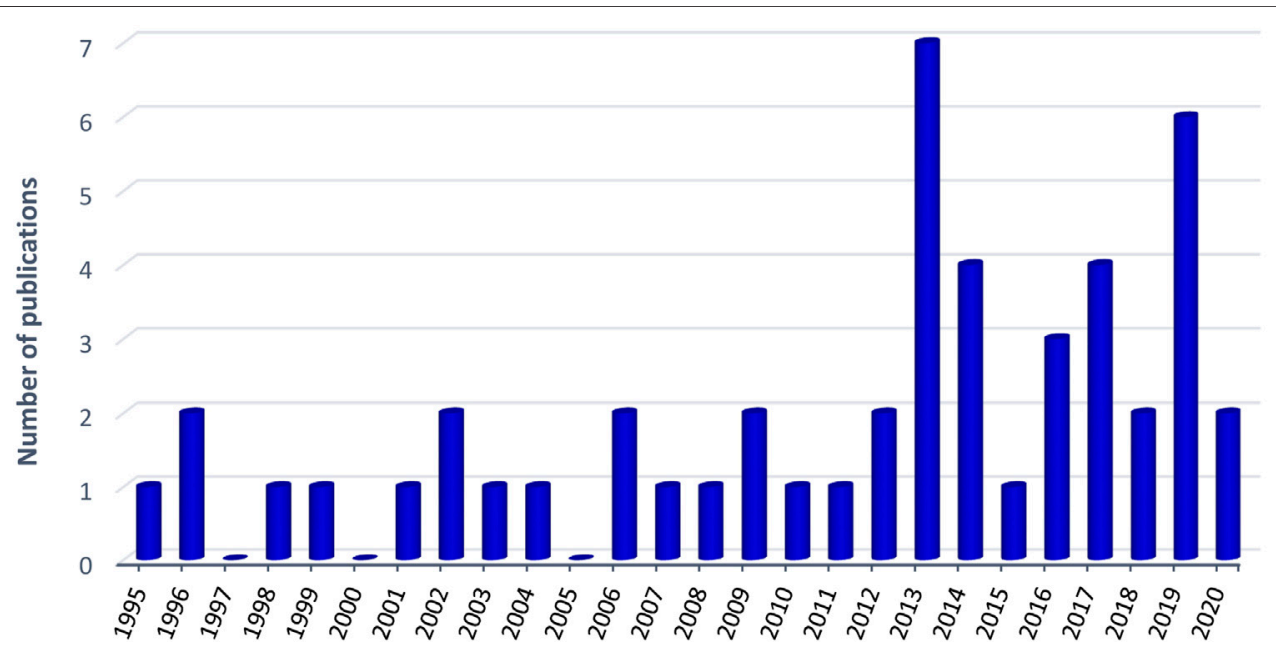

FIGURE 4 | Annual publication rate of Eriosema genus since 1995 (phytochemical, pharmacological and toxicological investigation and reviews). 
EEC and its subfractions did not cause any signs of toxicity or mortality up to $2 \mathrm{~g} / \mathrm{kg}$ during the observation period of 14 days (Prasad et al., 2013b).

In a study by Ateba et al. (2014a), the toxicity of a methanol extract of the aerial parts of E. laurentii following guidelines for acute (OECD guideline 423) and subchronic (OECD guideline 407) oral administrations was investigated. A single dose of $2 \mathrm{~g} / \mathrm{kg}$ of the extract as well as the repetition of the experiment, caused neither toxicological symptoms nor mortality and the $\mathrm{LD}_{50}$ was estimated $>5 \mathrm{~g} / \mathrm{kg}$. In 28-days repeated oral administration, no signs of toxicity were observed phenotypically and in the main organs of rats. The extract only induced a delayed decrease of relative spleen weight and reduced the white blood cell count in males at the highest dose of $400 \mathrm{mg} / \mathrm{kg}$.

Using the Irwin test, Prasad et al. (2017) showed that the acute oral administration of $\mathbf{8 1}$ (from the roots of E. chinense) up to $300 \mathrm{mg} / \mathrm{kg}$ did not result in any sign of behavioral/neurological toxicity or mortality during the observation period. The weight of organs was not affected. Some signs of physical toxicity were observed at the dose of $500 \mathrm{mg} / \mathrm{kg}$.

Nduka et al. (2018) reported $\mathrm{LD}_{50}$ values of $3807.9 \mathrm{mg} / \mathrm{kg}$ and $>5,000 \mathrm{mg} / \mathrm{kg}$ for ethanolic and aqueous leaf extracts of $E$. psoraleoides, respectively, following an acute oral treatment.

Toxicological evaluations are of major importance for medicinal plants and herbal products. Without these studies the estimation of their therapeutic potential is limited when the doses to induce non-fatal as well as fatal adverse effects are unknown. Based on the in vivo acute toxicity studies, the tested extracts of E. chinense, E. laurentii and E. psoraleoides are placed at category 5 (or unclassified) in the Globally Harmonized Classification System for Chemical Substances and Mixtures (GHS) as adopted by OECD (OECD, 2001). Such products with no deaths up to $2000 \mathrm{mg} / \mathrm{kg}$ (estimated $\mathrm{LD}_{50}>5000 \mathrm{mg} / \mathrm{kg}$ ) are considered practically non-toxic (Hodge and Sterner, 1949; Kennedy et al., 1986).

\section{CONCLUSION}

In this paper, we reviewed the available information concerning the traditional uses/ethnopharmacology, phytochemistry, pharmacology and toxicology of the genus Eriosema. Twenty-

\section{REFERENCES}

Abdelsamie, A. S., Salah, M., Siebenbürger, L., Merabet, A., Scheuer, C., Frotscher, M., et al. (2019). Design, synthesis, and biological characterization of orally active $17 \beta$-hydroxysteroid dehydrogenase type 2 inhibitors targeting the prevention of osteoporosis. J. Med. Chem. 62 (15), 7289-7301. doi:10.1021/ acs.jmedchem.9b00932

Adjanohoun, E., Ahyi, M. R. A., Ake Assi, L., Dramane, K., Elewude, J. A., Fadoju, S. O., et al. (1991). Contribution to ethnobotanical and floristic studies in Western Nigeria. Nigeria: CSTR-OUA, 420.

Adjanohoun, E. J., Adjakidje, V., Ahyi, M. R. A., Ake Assi, L., Akoegninou, A., d'Almeida, J., et al. (1989). Contribution aux études ethnobotaniques et five species ( $16.6 \%$ of the genus) recorded in the review are known as traditional medicines and used for nearly 121 ailments. It is evident from anectodical notes that the investigated numbers of species and traditionally treated health problems are so far lower than the reality. Moreover, around $75 \%$ of the traditional indications of these plants have not been studied yet in respective pre-clinical studies, indicating that more scientific investigations are necessary. Apart from data on ethnopharmacology, only 49 papers dealing with the phytochemical, pharmacological and toxicological investigation and reviews on Eriosema species and compounds occurring in this genus have been published in 25 years (Figure 4). The mean rate of approximately 2 publications per year is very low. Despite the high reputation of several species for curing or alleviating ED/impotence, especially in Africa, the investigation is only limited to two papers on E. kraussianum which have been published more than 15 years ago indicating an underexplored aspect that requires more research attention. Concerning phytochemistry, the paper covers a total of 107 compounds mainly belonging to the classes of flavonoids, chromones and terpenoids. The pharmacological activities of extracts and pure compounds from this genus focused on the management of erectile dysfunction, pre-eclamptic complications and anti-diabetic, estrogenic, hypolipidemic, anti-oxidant, anti-microbial, antiosteoporosis, anthelmintic, anti-diarrheal and anti-cancer effects as well as cyclooxygenase and acetylcholinesterase inhibitory properties. However, there is lack of studies dealing with the in-depth mechanisms involved in the observed activities in vitro and in animals. Moreover, no clinical study has been carried out till now. Beyond the pharmacological activities, the toxicity evaluation of medicinal plants is of high importance for their valorization. In vivo, acute toxicity carried out with $E$. chinense, E. laurentii and E. psoraleoides indicated a low toxicity of investigated extracts.

\section{AUTHOR CONTRIBUTIONS}

SA obtained literatures, wrote the first draft, and edited the manuscript; DN gave ideas and critically reviewed the manuscript; LK obtained the literatures, gave ideas, critically reviewed and edited the manuscript. All authors read and approved the manuscript.

floristiques en République populaire du Bénin. Agence de coopération culturelle et technique, (A.C.C.T.). Paris: Lagos, 895.

Adjanohoun, E. J., Adjakidje, V., Ahyi, M. R. A., Akpagana, K., Chibon, P., El-Hadji, A., et al. (1986). Contribution aux études ethnobotaniques et floristiques au Togo. Agence de coopération culturelle et technique, (A.C.C.T.). Paris: Lagos, 671.

Adjanohoun, E. J. (1993). Contribution to ethnobotanical and floristic studies in Uganda. O.U.A./C.S.T.R. Paris: Lagos.

Adomou, A., Yedomonhan, H., Djossa, B., Legba, S., Oumorou, M., and Akoegninou, A. (2012). Etude Ethnobotanique des plantes médicinales vendues dans le marché d'Abomey-Calavi au Bénin. Int. J. Bio. Chem. Sci. 6 (2), 745-772. doi:10.4314/ijbcs.v6i2.18

Akendengué, B., and Louis, A. M. (1994). Medicinal plants used by the Masango people in Gabon. J. Ethnopharmacology 41, 193-200. doi:10.1016/0378-8741(94)90032-9 
Ashraf, A. A., and Borthakur, S. K. (2005). Ethnobotanical wisdom of khasis (hynniewtreps) of Meghalaya. Dehradun: M/s Bishen Singh Mahendra Pal Singh Publication.

Ateba, S. B., Njamen, D., Gatterer, C., Scherzer, T., Zehl, M., Kählig, H., et al. (2016a). Rare phenolic structures found in the aerial parts of Eriosema laurentii De Wild. Phytochemistry 128, 5-11. doi:10.1016/j.phytochem.2016.03.020

Ateba, S. B., Njamen, D., Gatterer, C., Scherzer, T., Zehl, M., Kählig, H., et al. (2016b). Rare phenolic structures found in the aerial parts of Eriosema laurentii De Wildflavonoids from the underground parts of Eriosema laurentii. Phytochemistry. 128, 5-11. doi:10.1016/j.phytol.2016.10.00310.1016/j. phytochem.2016.03.020

Ateba, S. B., Njamen, D., Medjakovic, S., Hobiger, S., Mbanya, J. C., Jungbauer, A., et al. (2013). Eriosema laurentii De Wild (Leguminosae) methanol extract has estrogenic properties and prevents menopausal symptoms in ovariectomized Wistar rats. J. Ethnopharmacol. 150, 298-307. doi:10.1016/j.jep.2013.08.050

Ateba, S. B., Njamen, D., Medjakovic, S., Zehl, M., Kaehlig, H., Jungbauer, A., et al. (2014b). Lupinalbin A as the most potent estrogen receptor $a$ - and aryl hydrocarbon receptor agonist in Eriosema laurentii de Wild. (Leguminosae). BMC Complement. Altern. Med. 14, 294-303. doi:10.1186/1472-6882-14-294

Ateba, S. B., Simo, R. V., Mbanya, J. C., Krenn, L., and Njamen, D. (2014a). Safety profile and gender specific differences of a methanol extract of Eriosema laurentii (Leguminosae) in acute and subchronic (28 days) oral toxicity studies in Wistar rats. Food Chem. Toxicol. 65, 27-32. doi:10.1016/j.fct.2013.12.016

Ateba, S. B. (2014c). In vivo and in vitro estrogenic properties and safety profile Eriosema laurentii De Wild (Leguminosae) extracts. $\mathrm{PhD}$ Dissertation. Yaoundé; University of Yaoundé I.

Awouafack, M. D., Kouam, S. F., Hussain, H., Ngamga, D., Tane, P., Schulz, B., et al. (2008). Antimicrobial prenylated dihydrochalcones from Eriosema glomerata. Planta Med. 74, 50-54. doi:10.1055/s-2007-993782

Awouafack, M. D., McGaw, L. J., Gottfried, S., Mbouangouere, R., Tane, P., Spiteller, M., et al. (2013a). Antimicrobial activity and cytotoxicity of the ethanol extract, fractions and eight compounds isolated from Eriosema robustum (Fabaceae). BMC Complement. Altern. Med. 13, 289. doi:10.1186/ 1472-6882-13-289

Awouafack, M. D., Tane, P., Spiteller, M., and Eloff, J. N. (2015). Eriosema (Fabaceae) species represent a rich source of flavonoids with interesting pharmacological activities. Nat. Prod. Commun. 10 (7), 1325-1330. doi:10. 1177/1934578X1501000749

Awouafack, M. D., Tane, P., and Eloff, J. N. (2013b). Two new antioxidant flavones from the twigs of Eriosema robustum (Fabaceae). Phytochem. Lett. 6, 62-66. doi:10.1016/j.phytol.2012.10.017

Awouafack, M. D., Wong, C. P., Tane, P., and Morita, H. (2018). A new coumaronochromone and a new alkanoyl-dihydrofuranoflavone glycoside from Eriosema robustum (Fabaceae). Phytochem. Lett. 27, 20-24. doi:10.1016/j. phytol.2018.06.024

Awounfack, C. F., Ateba, S. B., Zingue, S., Mouchili, O. R., and Njamen, D. (2016). Safety evaluation (acute and sub-acute studies) of the aqueous extract of the leaves of Myrianthus arboreus P. Beauv. (Cecropiaceae) in Wistar rats. J. Ethnopharmacol. 194, 169-178. doi:10.1016/j.jep.2016.08.052

Aye, M., Aung, H., Sein, M., and Armijos, C. (2019). A review on the phytochemistry, medicinal properties and pharmacological activities of 15 selected Myanmar medicinal plants. Molecules 24, 293. doi:10.3390/ molecules 24020293

Baerts, M., and Lehmann, J. (1989). Guérisseurs et plantes médicinales de la région des crêtes Zaïre-Nil au Burundi. Tervuren, Belgique, Musée royal d'Afrique centrale (MRAC). Ann. Sci. économiques 18, 214.

Balouiri, M., Sadiki, M., and Ibnsouda, S. K. (2016). Methods for in vitro evaluating antimicrobial activity: a review. J. Pharm. Anal. 6, 71-79. doi:10.1016/j.jpha. 2015.11.00510.1016/j.jpha.2015.11.005

Barua, L., Faruque, M., Banik, P. C., and Ali, L. (2019). Atherogenic index of plasma and its association with cardiovascular disease risk factors among postmenopausal rural women of Bangladesh. Indian Heart J. 71 (2), 155-160. doi:10.1016/j.ihj.2019.04.012

Benfante, R., Di Lascio, S., Cardani, S., and Fornasari, D. (2019). Acetylcholinesterase inhibitors targeting the cholinergic antiinflammatory pathway: a new therapeutic perspective in aging-related disorders. Aging Clin. Exp. Res. 12, 133. doi:10.1007/s40520-01901359-4
Berman, A. N., and Blankstein, R. (2019). Optimizing dyslipidemia management for the prevention of cardiovascular disease: a focus on risk assessment and therapeutic options. Curr. Cardiol. Rep. 21, 110. doi:10.1007/s11886-019$1175-\mathrm{z}$

Bibi Sadeer, N., Montesano, D., Albrizio, S., Zengin, G., and Mahomoodally, M. F. (2020). The versatility of antioxidant assays in food science and safetychemistry, applications, strengths, and limitations. Antioxidants 9 (8), 709. doi:10.3390/antiox 9080709

Bossard, E. (1996). La medicine traditionnelle au centre et à l'ouest de l'Angola. Lisboa: Instituto de Investigação Científica Tropical.

Boulesteix, M., Guinko, S. C. A., and Libreville, M. E. S. (1979). Plantes médicinales utilisées par les Gbayas dans la région de Bouar (Empire Centrafricain). Quatrième colloque du Conseil africain de Malgache pour l'enseignement supérieur. Gabon, 23-52.

Bouquet, A. (1969). Féticheurs et médecines traditionnelles du Congo (Brazzaville). Paris: Féticheurs et médecines traditionnelles du Congo (Brazzaville), 282p.

Bryant, A. T. (1966). Zulu medicine and medicine-men. Cape Town: Centaur Struik.

Burkill, H. M. (1985). The useful plants of west tropical Africa. Editor Families, J. L. (Kew: Royal Botanic Garden).

Byavu, N., Henrard, C., Dubois, M., and Malaisse, F. (2000). Phytothérapie traditionnelle des bovins dans les élevages de la plaine de la Rusizi. Biotechnol. Agron. Soc. Environ. 4 (3), 135-156.

Calleja-Agius, J., and Brincat, M. P. (2015). The urogenital system and the menopause. Climacteric 18 (Suppl. 1), 18-22. doi:10.3109/13697137.2015.1078206

Cândido, E. S., De Vargas, W., Bezerra, L. M. D. P. A., Mansano, V. D. F., Vatanparast, M., Lewis, G. P., et al. (2019). Taxonomic synopsis of Eriosema (Leguminosae: Papilionoideae, Phaseoleae) in Brazil. Phytotaxa 416 (2), 91-137. doi:10.11646/phytotaxa.416.2.1

Cândido, E. S., Vatanparast, M., de Vargas, W., Bezerra, L. M. P. A., Lewis, G. P., Mansano, V. F., et al. (2020). Molecular phylogenetic insights into the evolution of Eriosema (Fabaceae): a recent tropical savanna-adapted genus. Bot. J. Linn. Soc. 194 (4), 439-459. doi:10.1093/botlinnean/boaa059

Chang, X., Yao, J., He, Q., Liu, M., Duan, T., and Wang, K. (2018). Exosomes from women with preeclampsia induced vascular dysfunction by delivering sFlt (soluble fms-like tyrosine kinase)-1 and sEng (soluble Endoglin) to endothelial cells. Hypertension 72 (6), 1381-1390. doi:10.1161/HYPERTENSIONAHA.118. 11706

Chen, S. L., Yu, H., Luo, H. M., Wu, Q., Li, C. F., and Steinmetz, A. (2016). Conservation and sustainable use of medicinal plants: problems, progress, and prospects. Chin. Med. 11, 37. doi:10.1186/s13020-016-0108-7

Cos, P., Hermans, N., De Bruyne, T., Apers, S., Sindambiwe, J. B., Vanden Berghe, D., et al. (2002b). Further evaluation of Rwandan medicinal plant extracts for their antimicrobial and antiviral activities. J. Ethnopharmacol. 79 (2), 155-163. doi:10.1016/s0378-8741(01)00362-2

Cos, P., Hermans, N., De, B. T., Apers, S., Sindambiwe, J. B., Witvrouw, M., et al. (2002a). Antiviral activity of Rwandan medicinal plants against human immunodeficiency virus type-1 (HIV-1). Phytomedicine 9, 62-68. doi:10. 1078/0944-7113-00083

Dalziel, J. M. (1937). The useful plants of west tropical Africa. The Crown Agents for the Colonies, 4, Millbank, London: Westminster, 612p.

de Morais, C. B., Scopel, M., Pedrazza, G. P. R., da Silva, F. K., Dalla Lana, D. F., Tonello, M. L., et al. (2017). Anti-dermatophyte activity of leguminosae plants from southern Brazil with emphasis on Mimosa pigra (leguminosae). J. Mycol. Med. 27 (4), 530-538. doi:10.1016/j.mycmed.2017.07.006

Descoings, B. (1963). Essai d'inventaire préliminaire des plantes médicinales d'Afrique Equatoriale. Bull. Inst. Rech. Scient. Congo, 2, 7-24.

Desouter, S. (1991). Pharmacopée humaine et vétérinaire du Rwanda. Musée royal de l'Afrique centrale Tervuren. Ann. Sc. Eco. 22, 254.

Dhir, A. (2019). An update of cyclooxygenase (COX)-inhibitors in epilepsy disorders. Expert Opin. Investig. Drugs 28 (2), 191-205. doi:10.1080/ 13543784.2019.1557147

Dobiášová, M. (2004). Atherogenic index of plasma [log(triglycerides/HDLcholesterol)]: theoretical and practical implications. Clin. Chem. 50, 1113-1115. doi:10.1373/clinchem.2004.033175

Dong, Y., Qiu, Q. Q., Debear, J., Lathrop, W. F., Bertolini, D. R., and Tamburini, P. P. (1998). 17Beta-hydroxysteroid dehydrogenases in human bone cells. J. Bone Miner. Res. 13, 1539-1546. doi:10.1359/jbmr.1998.13.10.1539 
Drewes, S. E., Horn, M. M., Khan, F., Munro, O. Q., Dhlamini, J. T., Rakuambo, C., et al. (2004). Minor pyrano-isoflavones from Eriosema kraussianum: activity-, structure-, and chemical reaction studies. Phytochemistry 65, 1955-1961. doi:10.1016/j.phytochem.2004.04.030

Drewes, S. E., Horn, M. M., Munro, O. Q., Dhlamini, J. T., Meyer, J. J., and Rakuambo, N. C. (2002). Pyrano-isoflavones with erectile-dysfunction activity from Eriosema kraussianum. Phytochemistry 59, 739-747. doi:10.1016/s00319422(02)00035-3

Drewes, S. E., Selepe, M. A., Van Heerden, F. R., Archer, R. H., and Mitchell, D. (2013). Unravelling the names, origins and chemistry of "muthis" used for male sexual disorders in KwaZulu-Natal, South Africa. South Afr. J. Bot. 88, 310-316. doi:10.1016/j.sajb.2013.08.010

Durand, J. M. (1960). Les plantes bienfaisantes du Ruanda et de l'Urundi. Astrida: Groupe scolaire, 89.

Eddy, A. C., Bidwell, G. L., 3rd, and George, E. M. (2018). Pro-angiogenic therapeutics for preeclampsia. Biol. Sex. Differ. 9 (1), 36. doi:10.1186/ s13293-018-0195-5

Edwards, M. K., Blaha, M. J., and Loprinzi, P. D. (2017). Atherogenic index of plasma and triglyceride/high-density lipoprotein cholesterol ratio predict mortality risk better than individual cholesterol risk factors, among an older adult population. Mayo Clin. Proc. 92 (4), 680-681. doi:10.1016/j.mayocp.2016. 12.018

Elechi, N. A., and Ewelike, F. W. (2019). Antidiabetic activity of fractions of the nhexane extract of leaves of Eriosema psoraleoides (lam) G. Don (leguminosae) on alloxan-induced diabetic albino rats. Ijpbr 7 (4), 05-09. doi:10.30750/ijpbr.7. 4.2

Elechi, N. A., and Igboh, O. T. (2017). Antibacterial activities of the methanol extract and fractions of the leaf of Eriosema psoraleoides (Lam.) G. Don (Leguminosae). Int. J. Pharma. Sci. Res. 8 (2), 698-705. doi:10.13040/IJPSR. 0975-8232.8(2).698-05

Eloff, J. N. (2004). Quantification the bioactivity of plant extracts during screening and bioassay guided fractionation. Phytomedicine 11, 370-371. doi:10.1078/ 0944711041495218

Eyre, L. J., Bland, R., Bujalska, I. J., Sheppard, M. C., Stewart, P. M., and Hewison, M. (1998). Characterization of aromatase and 17 beta-hydroxysteroid dehydrogenase expression in rat osteoblastic cells. J. Bone Miner. Res. 13, 996-1004. doi:10.1359/jbmr.1998.13.6.996

Fomogne-Fodjo, M. C., Van Vuuren, S., Ndinteh, D. T., Krause, R. W., and Olivier, D. K. (2014). Antibacterial activities of plants from Central Africa used traditionally by the Bakola pygmies for treating respiratory and tuberculosis-related symptoms. J. Ethnopharmacol. 155, 123-131. doi:10. 1016/j.jep.2014.04.032

Fumba, G. (1983). Plantes médicinales antivenimeuse du Burundi. Ministère du plan burundais. Imprimerie S.A. LES PRESSES DE L'AVENIR, 42, rue des Déportés, 6700 Arlon (Belgique).

Galabuzi, C., Nabanoga, G. N., Ssegawa, P., Obua, J., and Eilu, G. (2016). Responses to malaria incidence in the sango bay forest reserve, Uganda. Hum. Ecol. 44, 607-616. doi:10.1007/s10745-016-9855-4

Gillett, J. B., Polhill, R. M., and Verdcourt, B. (1971). “Papilionoideae (2)," in Flora of tropical East Africa, leguminosae. Editor E. Milne-Redhead. 4th ed. (London: Crown Agents for Overseas Governments and Administrations), 635-637.

Global Burden of Disease Study 2017; Causes of Death Collaborators (2018). Global, regional, and national age-sex-specific mortality for 282 causes of death in 195 countries and territories, 1980-2017: a systematic analysis for the Global Burden of Disease Study 2017 [published correction in Lancet 2019 Jun 22; 393 (10190): e44]. Lancet 392, 1736-1788. doi:10.1016/S0140-6736(18)32203-7

Göhre, A., Toto-Nienguesse, Á. B., Futuro, M., Neinhuis, C., Lautenschläge, T., and Lautenschläger, T. (2016). Plants from disturbed savannah vegetation and their usage by Bakongo tribes in Uíge, Northern Angola. J. Ethnobiol. Ethnomed. 12, 42. doi:10.1186/s13002-016-0116-9

Goossens, V. (1924). Catalogue des plantes du jardin botanique d'Eala (Congo Belge), Imprimerie industrielle et financière. Bruxelles: Ministère des colonies, direction de l'agriculture, V. Goossens, 179p.

Grosser, T., Theken, K. N., and FitzGerald, G. A. (2017). Cyclooxygenase inhibition: pain, inflammation, and the cardiovascular system. Clin. Pharmacol. Ther. 102 (4), 611-622. doi:10.1002/cpt.794

Hackett, G., Kirby, M., Wylie, K., Heald, A., Ossei-Gerning, N., Edwards, D., et al. (2018). British society for sexual medicine guidelines on the management of erectile dysfunction in men-2017. J. Sex. Med. 15, 430-457. doi:10.1016/j.jsxm. 2018.01.023

Haerdi, F. (1964). Native medicinal plants of ulanga district of tanganyika (East Africa). $\mathrm{PhD}$ dissertation. University of Basel.

Hastings, R. B. (1990). Medicinal legumes of Mexico: Fabaceae, papilionoideae, part one. Econ. Bot. 44, 336-348. doi:10.1007/BF03183915

Hatzimouratidis, K., Salonia, A., Adaikan, G., Buvat, J., Carrier, S., El-Meliegy, A., et al. (2016). Pharmacotherapy for erectile dysfunction: recommendations from the fourth international consultation for sexual medicine (ICSM 2015). J. Sex. Med. 13, 465-488. doi:10.1016/j.jsxm.2016.01.016

Haxaire, C. (1979). Phytothérapie et Médecine Familiale chez les Gbaya-Kara (République Centrafricaine. Thèse de doctorat, Université de Paris, Fac. Pharmacie., 320.

Hirschhorn, H. H. (1982). Botanical remedies of South and Central America, and the Caribbean: an archival analysis. Part II. Conclusion. J. Ethnopharmacol. 5, 163-180. doi:10.1016/0378-8741(82)90041-1

Hirschmann, G. S., and De Arias, A. R. (1990). A survey of medicinal plants of minas gerais. Brazil. J. Ethnopharmacol 29, 159-172. doi:10.1016/03788741(90)90052-U

Hodge, H. C., and Sterner, J. H. (1949). Tabulation of toxicity classes. Am. Ind. Hyg. Assoc. Q. 10, 93-96. doi:10.1080/00968204909344159

Hofer, U. (2019). The cost of antimicrobial resistance. Nat. Rev. Microbiol. 17 (1), 3. doi:10.1038/s41579-018-0125-x

Hulme, M. M. (1954). Wild flowers of natal. Pietermaritzburg: Schuter and Shooter.

Hutchings, A., Scott, A. H., Lewis, G., and Cunningham, A. (1996). Zulu medicinal plants: an inventory. Pietermaritzburg: University of Natal Press, 145.

Jena, M. K., Sharma, N. R., Petitt, M., Maulik, D., and Nayak, N. R. (2020). Pathogenesis of preeclampsia and therapeutic approaches targeting the placenta. Biomolecules 10 (6), 953. doi:10.3390/biom10060953

Jungbauer, A., and Beck, V. (2002). Yeast reporter system for rapid determination of estrogenic activity. J. Chromatogr. B Analyt Technol. Biomed. Life Sci. 777, 167-178. doi:10.1016/S1570-0232(02)00083-1

Kasonia, K., Basegere, N., Kaba, S., Matamba, M., and Katsongeri, M. (1991). Note sur les plantes vulnéraires et anti-inflammatoires employées en médecine vétérinaire traditionnelle au Zaïre oriental. Belg. Journ. Bot. 124 (1), 40-46.

Kennedy, G. L., Ferenz, R. L., and Burgess, B. A. (1986). Estimation of acute oral toxicity in rats by determination of the approximate lethal dose rather than the LD50. J. Appl. Toxicol. 6, 145-148. doi:10.1002/jat.2550060302

Kerharo, J., and Bouquet, A. (1950). Plantes médicinales et toxiques de la Côted'Ivoire-haute-Volta Mission d'étude de la pharmacopée indigène en A.O. Paris: .F. Editions Vigot Frères, 300.

Khan, M. N., Ngassapa, O., and Matee, M. I. (2000). Antimicrobial activity of Tanzanian chewing sticks against oral pathogenic microbes. Pharm. Biol. 38 (3), 235-240. doi:10.1076/1388-0209(200007)3831-SFT235

Kimpouni, V., Lenga-Sacadura, M.-Y., Mamboueni, J. C., and Mikoko, E. N. (2018). Phytodiversite et pharmacopée traditionnelle de La communauté kaamba de madingou (bouenza-Congo). Esj 14 (3), 191-220. doi:10.19044/ esj.2018.v14n3p191

Kisangau, D. P., Lyaruu, H. V., Hosea, K. M., and Joseph, C. C. (2007). Use of traditional medicines in the management of HIV/AIDS opportunistic infections in Tanzania: a case in the Bukoba rural district. J. Ethnobiol. Ethnomed. 3, 29. doi:10.1186/1746-4269-3-29

Kleynhans, R., Makena, I. M., and Matsiliza-Mlathi, B. (2020). "Eriosema kraussianum underexplored medicinal plants from sub-saharan Africa," in Plants with therapeutic potential for human health. Editor N. Lall (Academic Press), 99-104. doi:10.1016/B978-0-12-816814-1.00014-4

Kokwaro, J. O. (2009). Medicinal plants of East Africa. 3rd ed. Nairobi: University of Nairobi Press, 335.

Koné, W. M., Atindehou, K. K., Dossahoua, T., and Betschart, B. (2005). Anthelmintic activity of medicinal plants used in northern côte d'Ivoire against intestinal helminthiasis. Pharm. Biol. 43, 72-78. doi:10.1080/ 13880200590903408

Koné, W. M., Vargas, M., and Keiser, J. (2012). Anthelmintic activity of medicinal plants used in Côte d'Ivoire for treating parasitic diseases. Parasitol. Res. 110, 2351-2362. doi:10.1007/s00436-011-2771-z

Krzastek, S. C., Bopp, J., Smith, R. P., and Kovac, J. R. (2019). Recent advances in the understanding and management of erectile dysfunction. F1000Res. 8, 102. doi:10.12688/f1000research.16576.1 
Kuete, V. (2010). Potential of Cameroonian plants and derived products against microbial infections: a review. Planta Med. 76, 1479-1491. doi:10.1055/s-00301250027

Kunming Institute of Botany. (1979). Selection of Yunnan medicinal herbs, 1. Nairobi: University of Nairobi Press, 539.

Labrecque, M. P., Takhar, M. K., Hollingshead, B. D., Prefontaine, G. G., Perdew, G. H., and Beischlag, T. V. (2012). Distinct roles for aryl hydrocarbon receptor nuclear translocator and ah receptor in estrogen-mediated signaling in human cancer cell lines. PLoS One 7, e29545. doi:10.1371/journal.pone.0029545

Laloo, D., and Hemalatha, S. (2011). Ethnomedicinal plants used for diarrhea by tribals of Meghalaya, Northeast India. Pharmacogn Rev. 5 (10), 147-154. doi:10. 4103/0973-7847.91108

Lanini, J., Duarte-Almeida, J. M., Nappo, S. A., and Carlini, E. A. (2012). Are medicinal herbs safe? The opinion of plant vendors from Diadema (São Paulo, southeastern Brazil). Rev. Bras. Farmacogn. 22, 21-28. doi:10.1590/S0102695X2011005000188

Lautenschläger, T., Monizi, M., Pedro, M., Mandombe, J. L., Bránquima, M., Heinze, C., et al. (2018). First large-scale ethnobotanical survey in the province of Uíge, northern Angola. J. Ethnobiol. Ethnomed. 14, 51. doi:10.1186/s13002018-0238-3

Lawin, I. F., Lalèyè, F. O. A., Agbani, O. P., and Assogbadjo, A. E. (2015). Ethnobotanical assessment of the plant species used in the treatment of diabetes in the Sudano-Guinean zone of Benin. J. Anim. Plant Sci. 26 (3), 4108-4123.

Lemoine, E., and Thadhani, R. (2019). Affordable preeclampsia therapeutics. Trends Pharmacol. Sci. 40 (2), 85-87. doi:10.1016/j.tips.2018.12.007

Lemordant, D. (1972). Histoire et ethnobotanique du Kosso. Jatba 19 (12), 560-582. doi:10.3406/jatba.1972.3131

Lewalle, P. J., and Rodegem, F. M. (1968). Plantes Medicinales du Burundi. Q. J. Crude Drug Res. 8 (3), 1257-1270. doi:10.3109/13880206809108856

LPWG (The Legume Phylogeny Working Group). (2017). A new subfamily classification of the Leguminosae based on a taxonomically comprehensive phylogeny. Taxon 66, 44-77. 10.12705/661.3

Ma, W., Fukushi, Y., Ducrey, B., Hostettmann, K., and Tahara, S. (1999). Phenolic glycosides from Eriosema tuberosum. Phytochemistry 51, 1087-1093. doi:10. 1016/S0031-9422(99)00180-6

Ma, W. G., Fuzzati, N., Li, Q. S., Yang, C. R., Stoeckli-Evans, H., and Hostettmann, K. (1995). Polyphenols from Eriosema tuberosum. Phytochemistry 39, 1049-1061. doi:10.1016/0031-9422(95)00052-9

Ma, W. G., Fukushi, Y., Hostettmann, K., and Tahara, S. (1998). Isoflavonoid glycosides from Eriosema tuberosum. Phytochemistry 49, 251-254. doi:10.1016/ S0031-9422(97)00950-3

Ma, W. G., Fuzzati, N., Lu, S. L., Gu, D. S., and Hostettmann, K. (1996b). Further chromones from Eriosema tuberosum. Phytochemistry 43, 1339-1343. doi:10. 1016/S0031-9422(96)00494-3

Ma, W. G., Fuzzati, N., Xue, Y., Yangm, C. R., and Hostettmann, K. (1996a). Four chromones from Eriosema tuberosum. Phytochemistry 41 (5), 1287-1291. doi:10.1016/0031-9422(95)00732-6

Mabogo, D. E. N. (1990). The ethnobotany of the Vhavenda. Magister Scientiae in the Faculty of Science (Department of Botany). Thesis, University of Pretoria.

Malzy, P. (1954). Quelques plantes du Nord Cameroun et leurs utilisations. J. Agric. Trop. Bot. Appl. 1 (5-6), 148-179. doi:10.3406/jatba.1954.2147h

Marchais-Oberwinkler, S., Henn, C., Möller, G., Klein, T., Negri, M., Oster, A., et al. (2011). 17 $\beta$-Hydroxysteroid dehydrogenases (17 $\beta$-HSDs) as therapeutic targets: protein structures, functions, and recent progress in inhibitor development. J. Steroid Biochem. Mol. Biol. 125, 66-82. doi:10.1016/j.jsbmb. 2010.12.013

Marconett, C. N., Sundar, S. N., Poindexter, K. M., Stueve, T. R., Bjeldanes, L. F., and Firestone, G. L. (2010). Indole-3-carbinol triggers aryl hydrocarbon receptor-dependent estrogen receptor (ER)alpha protein degradation in breast cancer cells disrupting an ERalpha-GATA3 transcriptional crossregulatory loop. Mol. Biol. Cel. 21, 1166-1177. doi:10.1091/mbc.e09-08-0689

Martin, P., and Ryan, B. (2004). Natural-series radionuclides in traditional aboriginal foods in tropical northern Australia: a review. ScientificWorldJournal 4, 77-95. doi:10.1100/tsw.2004.6

Masinde, P. S. (1996). "Medicinal plants of the Marachi people of Kenya," in The proceedings of the 14th AETFAT congres wageningen, The Netherlands 22-27 august 1996. Editors van der Maesen, J. G., van der Burgt, X. M., and van Medenbach de Rooy, J. M. (London: Kluwer Academic Publishers), 747-750.

Matias, J. R., Martin, J. L., and Burns, T. W. (1978). Ricinoleic acid effect on the electrical activity of the small intestine in rabbits. J. Clin. Invest. 61, 640-644. doi:10.1172/JCI108975

Mayrink, J., Costa, M. L., and Cecatti, J. G. (2018). Preeclampsia in 2018: revisiting concepts, physiopathology, and prediction. Scientific World J. 21, 1. doi:10. $1155 / 2018 / 6268276$

McMahon, C. G. (2019). Current diagnosis and management of erectile dysfunction. Med. J. Aust. 210 (10), 469-476. doi:10.5694/mja2.50167

Michos, E. D., McEvoy, J. W., and Blumenthal, R. S. (2019). Lipid management for the prevention of atherosclerotic cardiovascular disease. N. Engl. J. Med. 381 (16), 1557-1567. doi:10.1056/NEJMra1806939

Mmbengwa, V., Samie, A., Gundidza, M., Matikiti, V., Ramalivhana, N. J., and Magwa, M. L. (2009). Biological activity and phytoconstituents of essential oil from fresh leaves of Eriosema englerianum. Afr. J. Biotechnol. 8, 361-364. doi:10.5897/ajb09.430

Morton, J. F. (1981). "Atlas of medicinal plants of Middle America: Bahamas to Yucatan,”. Editor C. C. Thomas (Springfield, IL: Springer), 316.

Moses, R. A., Anderson, R. E., Kim, J., Keihani, S., Craig, J. R., Myers, J. B., et al. (2019). Erectile dysfunction management after failed phosphodiesterase-5inhibitor trial: a cost-effectiveness analysis. Transl. Androl. Urol. 8 (4), 387-394. doi:10.21037/tau.2019.03.1010.21037/tau.2019.03.10

Moshi, M. J., Otieno, D. F., Mbabazi, P. K., and Weisheit, A. (2010). Ethnomedicine of the kagera region, north western Tanzania. Part 2: the medicinal plants used in katoro ward, bukoba district. J. Ethnobiol. Ethnomed. 6, 19. doi:10.1186/ 1746-4269-6-19

Moshi, M. J., Otieno, D. F., Mbabazi, P. K., and Weisheit, A. (2009). The ethnomedicine of the Haya people of Bugabo ward, Kagera Region, north western Tanzania. J. Ethnobiol. Ethnomed. 5 (5), 24. doi:10.1186/1746-42695-24

Msonthi, J. D., and Magombo, D. (1983). Medicinal herbs in Malawi and their uses. Hamdard Medicus 26, 94-100.

Mvondo, M. A., Njamen, D., Fomum, S. T., Wandji, J., and Vollmer, G. (2011). A postmenopause-like model of ovariectomized Wistar rats to identify active principles of Erythrina lysistemon (Fabaceae). Fitoterapia 82, 939-949. doi:10. 1016/j.fitote.2011.05.009

Nappi, R. E., and Cucinella, L. (2020). "Long-term consequences of menopause," in Female reproductive dysfunction, endocrinology. Editors F. Petraglia and B. Fauser (Cham: Springer), 1-13. doi:10.1007/978-3-030-03594-5_17-1

Nasri, H., and Shirzad, H. (2013). Toxicity and safety of medicinal plants. J. Herbmed Pharmacol. 2 (2), 21-22. doi:10.1201/9781482293982-9

Ndhlala, A. R., Finnie, J. F., and Van Staden, J. (2011). Plant composition, pharmacological properties and mutagenic evaluation of a commercial Zulu herbal mixture: Imbiza ephuzwato. J. Ethnopharmacol. 133, 663-674. doi:10. 1016/j.jep.2010.10.053

Nduka, F. O., Ogugua, V. N., Joshua, P. E., Okpachi, V. E., Gometi, S. A., and Nwigwe, J. O. (2018). Anti-diabetic and some haematological effects of aqueous and ethanol leaf extract of Eriosema psoraleoides in alloxan-induced diabetic Wistar rats. Afr. J. Biotechnol. 17 (41), 1292-1298. doi:10.5897/AJB2018.16603

Nduka, F. O., Ogugua, V. N., Nwigwe, J. O., Nwaso, C. B., and Abdulrasheed, M. B. (2019). Effect of aqueous leaf extract of Eriosema psoraleoides on antihyperglycemic and hypolipidemic potentials in alloxan-induced diabetic rats. Asian J. Res. Biochem. 4 (1), 1-8. doi:10.9734/AJRB/2019/v4i130061

Neogi, B., Prasad, M. N. V., and Rao, R. R. (1989). Ethnobotany of some weeds of khasi and garo hills, Meghalaya, northeastern India. Econ. Bot. 43, 471-479. doi:10.1007/BF02935921

Ngbolua, K-N., Bongo, G. N., Ashande, M. C., Djoza, D. R., Mpiana, P. T., Mudogo, V., et al. (2014). Ethno-botanical survey and ecological study of plants resources used in folk medicine to treat symptoms of tuberculosis in kinshasa city, democratic republic of the Congo. J. Mod. Drug Discov. Drug Deliv. Res. 1 (4), 6. doi:10.22271/tpr.2017.v4.i3.048

Ngezahayo, J., Havyarimana, F., Hari, L., Stévigny, C., and Duez, P. (2015). Medicinal plants used by Burundian traditional healers for the treatment of microbial diseases. J. Ethnopharmacol. 173, 338-351. doi:10.1016/j.jep.2015. 07.028

Nondo, R. S. O., Zofou, D., Moshi, M. J., Erasto, P., Wandji, S., Ngemenya, M. N., et al. (2015). Ethnobotanical survey and in vitro antiplasmodial activity of 
medicinal plants used to treat malaria in Kagera and Lindi regions, Tanzania. J. Med. Plants Res. 9 (6), 179-192. doi:10.5897/JMPR2014.5685

Novotna, B., Polesny, Z., Pinto-Basto, M. F., Van Damme, P., Pudil, P., Mazancova, J., et al. (2020). Medicinal plants used by 'root doctors', local traditional healers in Bié province, Angola. J. Ethnopharmacol. 260, 112662. doi:10.1016/j.jep. 2020.112662

Nyakabwa, M., and Gapusi, R. (1990). Plantes médicinales utilisées chez les Banyamulenge de Fizi au Sud-Kivu (Zaïre). Afr. Stud. Monogr. 11 (2), 101-114. doi:10.1007/978-94-009-0285-5_92

OECD (Organization for Economic Cooperation and Development). (2001). Harmonised integrated classification system for human health and environmental hazards of chemical substances and mixtures. Chapter 2.1, adopted 14th August 2001. Paris: OECD.

OECD (Organization for Economic Cooperation and Development). (2007). OECD Guideline for Testing of Chemicals. Test No. 440: Uterotrophic Bioassay in Rodents: A Short-Term Screening Test for Oestrogenic Properties. Adopted 16th October 2007. Paris: OECD Environement Directorate.

Ojewole, J. A., Drewes, S. E., and Khan, F. (2006). Vasodilatory and hypoglycaemic effects of two pyrano-isoflavone extractives from Eriosema kraussianum N. E. Br. [Fabaceae] rootstock in experimental rat models. Phytochemistry 67, 610-617. doi:10.1016/j.phytochem.2005.11.019

Ojewole, J. A. O., and Drewes, S. E. (2007). Hypoglycaemic effect of Eriosema kraussianum N. E. Br. [Fabaceae] rootstock hydro-alcohol extract in rats. J. Nat. Med. 61, 244-250. doi:10.1007/s11418-006-0129-0

Okino, S. T., Pookot, D., Basak, S., and Dahiya, R. (2009). Toxic and chemopreventive ligands preferentially activate distinct aryl hydrocarbon receptor pathways: implications for cancer prevention. Cancer Prev. Res. (Phila) 2, 251-256. doi:10.1158/1940-6207.CAPR-08-0146

Ouoba, P., Lykke, A. M., Boussim, J., and Guinko, S. (2006). La flore médicinale de la Foret Classée de Niangoloko (Burkina Faso). Etudes Flor. Vég. Burkina Faso 10, 5-16. doi:10.1051/fruits:2006006

Oyston, C., and Baker, P. N. (2020). Current challenges in pregnancy-related mortality. Obstet. Gynaecol. Reprod. Med. 30 (2), 55-61. doi:10.1016/j.ogrm. 2019.11.003

Parmar, K. M., Bhagwat, D. S., Sinha, S. K., Katare, N. T., and Prasad, S. K. (2019b). The potency of eriosematin E from Eriosema chinense Vogel. against enteropathogenic Escherichia coli induced diarrhoea using preclinical and molecular docking studies. Acta Trop. 193, 84-91. doi:10.1016/j.actatropica. 2019.02.025

Parmar, K. M., Hirudkar, J. R., Bhagwat, D. S., and Prasad, S. K. (2019a). Antidiarrheal potential of Eriosema chinense Vogel. against enteropathogenic Escherichia coli-induced infectious diarrhea. Pharmacogn. Mag. 15, S455-S4561. doi:10.4103/pm.pm_129_19

Pisani, P., Renna, M. D., Conversano, F., Casciaro, E., Di Paola, M., Quarta, E., et al. (2016). Major osteoporotic fragility fractures: risk factor updates and societal impact. World J. Orthop. 7 (3), 171-181. doi:10.5312/wjo.v7.i3.171

Polygenis-Bigendako, M-J. (1990). Recherches ethnopharmacognosiques sur les plantes utilisées en médecine traditionnelle au Burundi Occidental. Thèse de Doctorat, Université Libre de Bruxelles, 352.

Porst, H., Burnett, A., Brock, G., Ghanem, H., Giuliano, F., Glina, S., et al. (2013). SOP conservative (medical and mechanical) treatment of erectile dysfunction. J. Sex. Med. 10 (1), 130-171. doi:10.1111/jsm.12023

Prasad, S., Jain, D., Kumar, M., and Hemalatha, S. (2013b). Antioxidant and antibacterial potential of different fractions from roots of Eriosema chinense Vogel. Bjpr 3, 135-146. doi:10.9734/BJPR/2013/2661

Prasad, S. K., Laloo, D., Kumar, M., and Hemalatha, S. (2013c). Antidiarrhoeal evaluation of root extract, its bioactive fraction, and lupinifolin isolated from Eriosema chinense. Planta Med. 79, 1620-1627. doi:10.1055/s-00331351021

Prasad, S. K., Parmar, K. M., Danta, C. C., Laloo, D., and Hemalatha, S. (2017). Antidiarrhoeal activity of eriosematin $\mathrm{E}$ isolated from the roots of Eriosema chinense Vogel. Phytomedicine 24, 127-133. doi:10.1016/j.phymed.2016.11.022

Prasad, S. K., Laloo, D., Kumar, M., and Hemalatha, S. (2013a). Quality control standardization and antioxidant activity of roots from Eriosema chinense. Pharmacognosy J. 5, 149-155. doi:10.1016/j.phcgj.2013.07.007

Racusen, L. C., and Binder, H. J. (1979). Ricinoleic acid stimulation of active anion secretion in colonic mucosa of the rat. J. Clin. Invest. 63, 743-749. doi:10.1172/ JCI109358
Ramesar, S. V., Drewes, S. E., Gathiram, P., Moodley, J., and Mackraj, I. (2012). The effect of Kraussianone-2 (Kr2), a natural pyrano-isoflavone from Eriosema kraussianum, in an L-NAME- induced pre-eclamptic rat model. Phytother. Res. 26, 1375-1380. doi:10.1002/ptr.3697

Rana, S., Lemoine, E., Granger, J. P., and Karumanchi, S. A. (2019). Preeclampsia: pathophysiology, challenges, and perspectives. Circ. Res. 124, 1094-1112. doi:10.1161/CIRCRESAHA.118.313276

Reiter, E., Beck, V., Medjakovic, S., Mueller, M., and Jungbauer, A. (2009). Comparison of hormonal activity of isoflavone-containing supplements used to treat menopausal complaintsflavone-containing supplements used to treat menopausal complaints. Menopause 16, 1049-1060. doi:10.1097/gme. ob013e31819c146c

Rendell, M. S., Rajfer, J., Wicker, P. A., and Smith, M. D. (1999). Sildenafil for treatment of erectile dysfunction in men with diabetes: a randomized controlled trial. Sildenafil Diabetes Study Group. JAMA 281, 421-426. doi:10.1001/jama. 281.5.421

Ríos, J. L., and Recio, M. C. (2005). Medicinal plants and antimicrobial activity. J. Ethnopharmacology 100, 80-84. doi:10.1016/j.jep.2005.04.025

Rodrigues, E. (2007). Plants of restricted use indicated by three cultures in Brazil (Caboclo-river dweller, Indian and Quilombola). J. Ethnopharmacol. 111 (2), 295-302. doi:10.1016/j.jep.2006.11.017

Runyoro, D. K., Matee, M. I., Ngassapa, O. D., Joseph, C. C., and Mbwambo, Z. H. (2006). Screening of Tanzanian medicinal plants for anti-Candida activity. BMC Complement. Altern. Med. 6, 11. doi:10.1186/1472-6882-6-11

Rwangabo, P. C. (1993). La Me'decine Traditionnelle au Rwanda. Paris: Editions Karthala et ACCT.

Sandberg, F. (1965). Etude sur les plantes médicinales et toxiques de l'Afrique équatoriale. Tome: Cahiers de la Maboké, 5-49.

Santos, M. G., Almeida, V. G., Avelar-Freitas, B. A., Grael, C. F. F., Gregório, L. E., Pereira, W. F., et al. (2016). Phytochemical screening of the dichloromethaneethanolic extract of Eriosema campestre var. macrophylum roots and its antiproliferative effect on human peripheral blood lymphocytes. Rev. Bras. Farmacogn. 26, 464-470. doi:10.1016/j.bjp.2015.08.009

Schrire, B. D. (2005). "Tribe phaseoleae," in Legumes of the world. Editors Lewis, G. P., B. Schrire, B. Mackinder, and M. Lock (Richmond, U.K.Kew: The Royal Botanic Gardens), 393-431.

Sianglum, W., Muangngam, K., Joycharat, N., and Voravuthikunchai, S. P. (2019). Mechanism of action and biofilm inhibitory activity of lupinifolin against multidrug-resistant enterococcal clinical isolatesfilm inhibitory activity of lupinifolin against multidrug-resistant enterococcal clinical isolates. Microb. Drug Resist. 25 (10), 1391-1400. doi:10.1089/mdr.2018.03910.1089/mdr.2018. 0391

Sillans, R. (1953). Medicinal plants of Central Africa II. Annales pharmaceutiques franchaises 11, 456.

Ssegawa, P., and Kasenene, J. M. (2007). Medicinal plant diversity and uses in the Sango bay area, Southern Uganda. J. Ethnopharmacol. 113, 521-540. doi:10. 1016/j.jep.2007.07.014

Staner, P., and Boutique, R. (1937). Matériaux pour l'étude des plantes médicinales indigènes du Congo Belge. Mémoire Institut royal colonial belge, Section des Sc. naturelles et médicales, Collection in-8, fasc. 6 et dernier, 228 p.

Sutthivaiyakit, S., Thongnak, O., Lhinhatrakool, T., Yodchun, O., Srimark, R., Dowtaisong, P., et al. (2009). Cytotoxic and antimycobacterial prenylated flavonoids from the roots of Eriosema chinense. J. Nat. Prod. 72, 1092-1096. doi:10.1021/np900021h

Tacconelli, E., and Pezzani, M. D. (2019). Public health burden of antimicrobial resistance in Europe. Lancet Infect. Dis. 19 (1), 4-6. doi:10.1016/S14733099(18)30648-0

Tan, J. B., and Lim, Y. Y. (2015). Critical analysis of current methods for assessing the in vitro antioxidant and antibacterial activity of plant extracts. Food Chem. 172, 814-822. doi:10.1016/j.foodchem.2014.09.141

Taylor, D. G., Giuliano, F., Hackett, G., Hermes-DeSantis, E., Kirby, M. G., Kloner, R. A., et al. (2019). The pharmacist's role in improving the treatment of erectile dysfunction and its underlying causes. Res. Soc. Administrative Pharm. 15, 591-599. doi:10.1016/j.sapharm.2018.07.014

Thongnest, S., Lhinhatrakool, T., Wetprasit, N., Sutthivaiyakit, P., and Sutthivaiyakit, S. (2013). Eriosema chinense: a rich source of antimicrobial and antioxidant flavonoids. Phytochemistry 96, 353-359. doi:10.1016/j. phytochem.2013.06.004 
Tsuchiya, Y., Nakajima, M., and Yokoi, T. (2005). Cytochrome P450-mediated metabolism of estrogens and its regulation in human. Cancer Lett. 227, 115-124. doi:10.1016/j.canlet.2004.10.007

Van Puyvelde, L., Ngaboyisonga, M., Rwangabo, P. C., Mukarugambwa, S., Kayonga, A., and Runyinya-Barabwiriza. (1977). Enquêtes ethnobotaniques sur la médecine traditionnelle rwandaise. Tome 1: Préfecture de Kibuye. Butare (Rwanda): Université Nationale du Rwanda et l'Institut National de Recherche Scientifique, $147 \mathrm{p}$.

Van Wyk, B.-E., Van Oudtshoorn, B., and Gericke, N. (1997). Medicinal plants of South Africa. Pretoria: Briza Publications.

Verdcourt, B. (1970). Studies in the leguminosae-papilionoideae for the 'flora of tropical East Africa': I. Kew Bull. 24 (1), 1-70. doi:10.2307/4103248

Verger, P. F. (1995). Ewé: the use of plants in Yoruba society. Sao Paulo: Editoria Schwarcz, 744p.

Vergiat, A. M. (1969). Plantes magiques et médicinales des féticheurs de l'Oubangui (Région de Bangui) (3e partie). J. Agric. Trop. Bot. Appl. 16 (910), 418-456. doi:10.3406/jatba.1969.3037

Vuorinen, A., Engeli, R. T., Leugger, S., Bachmann, F., Akram, M., Atanasov, A. G., et al. (2017). Potential antiosteoporotic natural product lead compounds that inhibit 17 $\beta$-hydroxysteroid dehydrogenase type 2. J. Nat. Prod. 80 (4), 965-974. doi:10.1021/acs.jnatprod.6b00950

Wanyama, P. J. (2010). Phytochemical investigation of Tephrosia purpurea and Eriosema psoraleoides for pharmacological and antiplasmodial activities. Master of Science Dissertation, University of Nairobi.

Watt, J. M., and Breyer-Brandwijk, M. G. (1962). The medicinal and poisonous plants of Southern and Eastern Africa. Second ed. Edinburgh and London: E. \& S Livingstone Ltd., 600-601.
Wome, B. (1985). Recherches ethnopharmacognosiques sur les plantes médicinales utilisées en médecine traditionnelle à Kisangani (Haut-Zaïre). Thèse de Doctorat,Université Libre de Bruxelles, 561.

Wu, T. T., Gao, Y., Zheng, Y. Y., Ma, Y. T., and Xie, X. (2018). Atherogenic index of plasma (AIP): a novel predictive indicator for the coronary artery disease in postmenopausal women. Lipids Health Dis. 17 (1), 197. doi:10.1186/s12944-018-0828-Z

Wu, Z. Y. (1991). The areal-types of Chinese genera of seed plants. Acta Botanica Yunn. Supp. IV 12, 1-139.

Yafi, F. A., Sharlip, I. D., and Becher, E. F. (2018). Update on the safety of phosphodiesterase type 5 inhibitors for the treatment of erectile dysfunction. Sex. Med. Rev. 6 (2), 242-252. doi:10.1016/j.sxmr.2017.08.001

Yuan, J., Zhang, R., Yang, Z., Lee, J., Liu, Y., Tian, J., et al. (2013). Comparative effectiveness and safety of oral phosphodiesterase type 5 inhibitors for erectile dysfunction: a systematic review and network meta-analysis. Eur. Urol. 63, 902-912. doi:10.1016/j.eururo.2013.01.012

Conflict of Interest: The authors declare that the research was conducted in the absence of any commercial or financial relationships that could be construed as a potential conflict of interest.

Copyright (c) 2021 Ateba, Njamen and Krenn. This is an open-access article distributed under the terms of the Creative Commons Attribution License (CC $B Y)$. The use, distribution or reproduction in other forums is permitted, provided the original author(s) and the copyright owner(s) are credited and that the original publication in this journal is cited, in accordance with accepted academic practice. No use, distribution or reproduction is permitted which does not comply with these terms. 